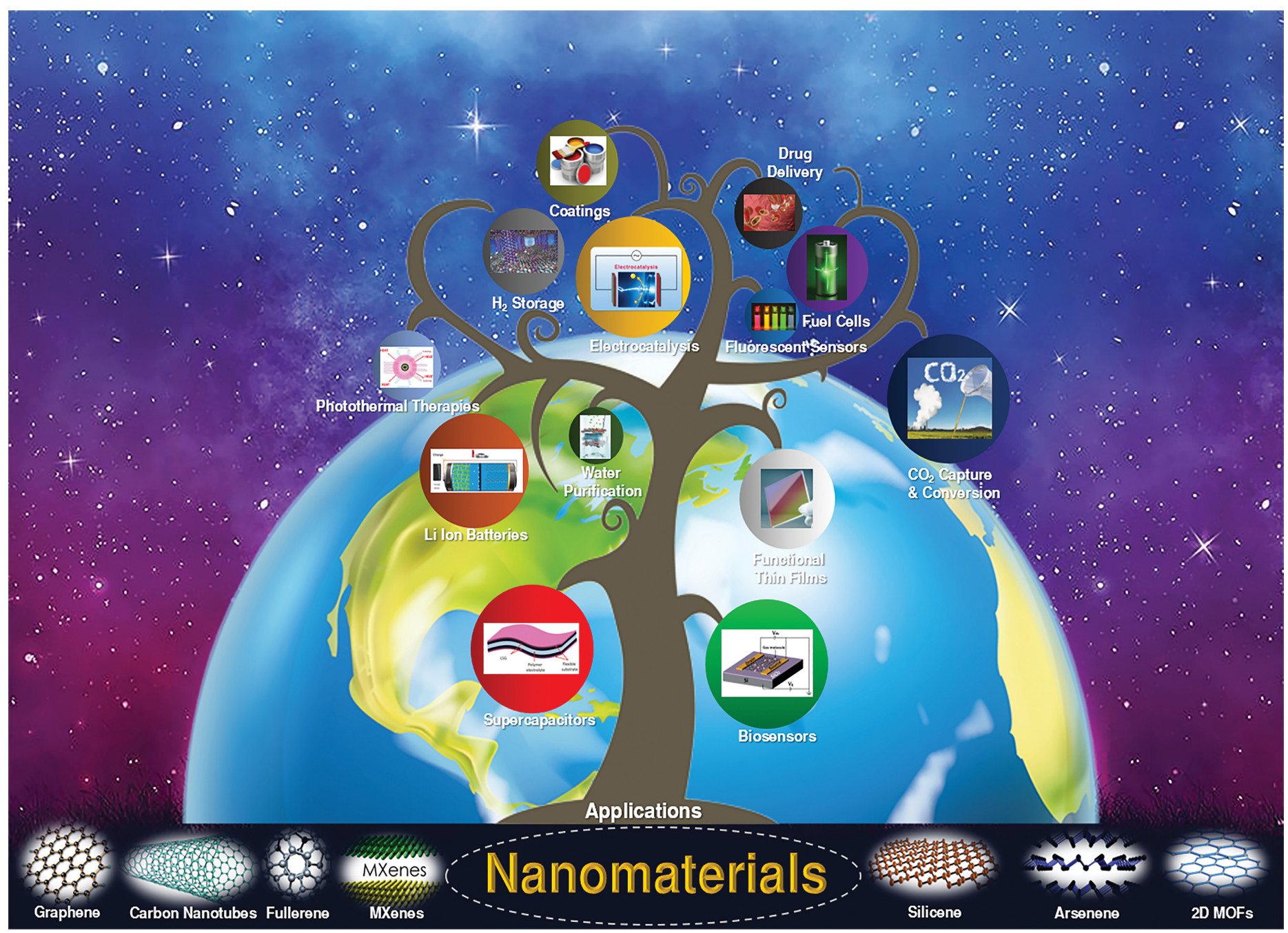

Showcasing research from Dr Nadeem Baig and Dr Wail Sulaiman Falath laboratory at Center of Research Excellence in Desalination \& Water Treatment, King Fahd University of Petroleum and Minerals, Dhahran, Saudi Arabia.

Nanomaterials: a review of synthesis methods, properties, recent progress, and challenges

Dr Baig's group is working on the development of various advanced nanomaterials for a range of applications. They are deeply interested in developing nanocomposite membranes with enhanced performance for desalination. In this article, Dr Baig, Dr Irshad, and Dr Wail have discussed nanomaterials' advancements and their effectiveness for various applications. Various top-down and bottom-up approaches are discussed to synthesize the nanomaterials. This article has highlighted some critical challenges in producing defect-free nanomaterials that result in compromising their unique features.
As featured in:

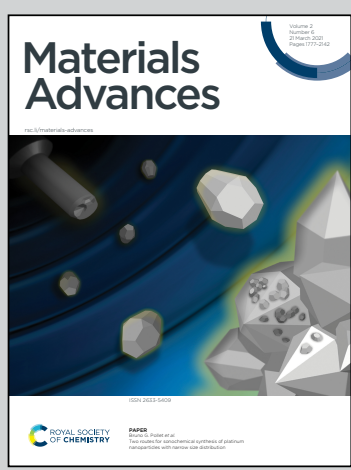

See Nadeem Baig,

Irshad Kammakakam et al., Mater. Adv., 2021, 2, 1821. 
Check for updates

Cite this: Mater. Adv., 2021 2, 1821

Received 17th October 2020 Accepted 23rd February 2021

DOI: 10.1039/d0ma00807a

rsc.li/materials-advances

\section{Nanomaterials: a review of synthesis methods, properties, recent progress, and challenges}

\begin{abstract}
Nadeem Baig, (D)*abc Irshad Kammakakam (D) *d and Wail Falath ${ }^{\text {abe }}$
Nanomaterials have emerged as an amazing class of materials that consists of a broad spectrum of examples with at least one dimension in the range of 1 to $100 \mathrm{~nm}$. Exceptionally high surface areas can be achieved through the rational design of nanomaterials. Nanomaterials can be produced with outstanding magnetic, electrical, optical, mechanical, and catalytic properties that are substantially different from their bulk counterparts. The nanomaterial properties can be tuned as desired via precisely controlling the size, shape, synthesis conditions, and appropriate functionalization. This review discusses a brief history of nanomaterials and their use throughout history to trigger advances in nanotechnology development. In particular, we describe and define various terms relating to nanomaterials. Various nanomaterial synthesis methods, including top-down and bottom-up approaches, are discussed. The unique features of nanomaterials are highlighted throughout the review. This review describes advances in nanomaterials, specifically fullerenes, carbon nanotubes, graphene, carbon quantum dots, nanodiamonds, carbon nanohorns, nanoporous materials, core-shell nanoparticles, silicene, antimonene, MXenes, 2D MOF nanosheets, boron nitride nanosheets, layered double hydroxides, and metal-based nanomaterials. Finally, we conclude by discussing challenges and future perspectives relating to nanomaterials.
\end{abstract}

\footnotetext{
${ }^{a}$ Center of Research Excellence in Desalination \& Water Treatment, King Fahd University of Petroleum and Minerals, Dhahran 31261, Saudi Arabia. E-mail: nadeembaig@kfupm.edu.sa,nadeembaig38@gmail.com

${ }^{b}$ Center for Environment and Water, King Fahd University of Petroleum and Minerals, Dhahran 31261, Saudi Arabia

${ }^{c}$ Interdisciplinary Research Center for Membranes and Water Security, King Fahd University of Petroleum and Minerals, Dhahran 31261, Saudi Arabia

${ }^{d}$ Department of Chemical \& Biological Engineering, University of Alabama, Tuscaloosa, Alabama 35487-0203, USA. E-mail: irshadk31@gmail.com, ikammakakam@eng.ua.edu

${ }^{e}$ Department of Mechanical Engineering, King Fahd University of Petroleum and Minerals, Dhahran 31261, Saudi Arabia
}

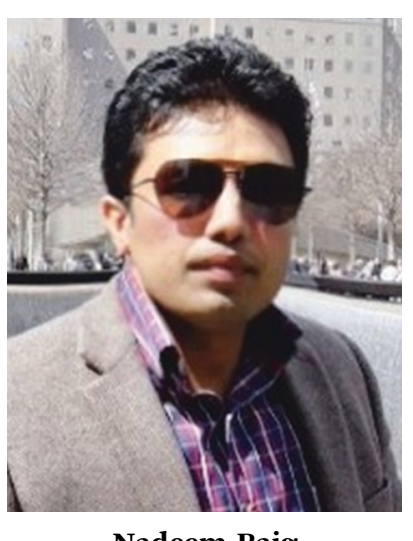

Nadeem Baig
Dr Nadeem Baig received his BS (Hons.) in 2008 from the University of the Punjab and MPhil from the University of Engineering and Technology, Lahore, in 2012. In 2017, he received his $P h D$ degree from KFUPM, Saudi Arabia. After working as a postdoctoral researcher for two years, Dr Baig, in 2020 has joined the Center of Research Excellence in Desalination \& Water Treatment at KFUPM as a Research Scientist III. He is currently working at IRC for Membranes and Water Security. His research focused on developing nanostructured and functionalized nanomaterials for sensors, energy, water, and environmental applications.

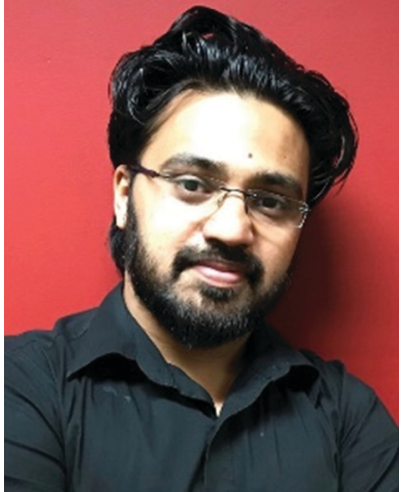

Irshad Kammakakam
Dr Irshad Kammakakam is a postdoctoral research scholar at the University of Alabama. He works on the development of advanced high-performance hybrid polymers as gas separation membranes. He received his $P h D$ in Chemistry from Incheon National University in 2015, focusing on developing functional polymer membranes for gas separation applications. Later, he worked as a Visiting Scientist at the Korea Institute of Science and Technology and completed a Postdoc at the European Institute of Membranes, the University of Montpellier, France. His research interest is to develop new advanced polymeric materials for separation technology and environmental green energy applications. 


\section{Introduction}

Nanomaterials have emerged as an exciting class of materials that are in high demand for a range of practical applications. The length of a nanometer can be understood through the example of five silicon atoms or 10 hydrogen atoms lined up, which is one nanometer. Materials are defined as nanomaterials if their size or one of their dimensions is in the range of 1 to $100 \mathrm{~nm}$. The exact history of the utilization of nanosized objects by humans is difficult to clarify. However, the history of nanomaterial utilization is ancient, and human beings used these materials a long time ago for various applications, unknowingly. About 4500 years ago, humans exploited asbestos nanofibers to reinforce ceramic mixtures. ${ }^{1}$ The ancient Egyptians were familiar with $\mathrm{PbS}$ nanoparticles about 4000 years ago and used them in an ancient hair-dyeing formula., ${ }^{2,3}$ The Lycurgus Cup is another fascinating example from the past. It is a dichroic cup produced by the Romans in the 4 th century A.D. It resembles jade in direct light, whereas it shows a translucent ruby color in the case of transmitted light. It shows color variations depending on the incident light. These color variations appear due to the presence of nanoparticles of $\mathrm{Ag}$ and $\mathrm{Au}^{4}$

The term nanometer was first used in 1914 by Richard Adolf Zsigmondy. ${ }^{5}$ The American physicist and Nobel Prize laureate Richard Feynman introduced the specific concept of nanotechnology in 1959 in his speech during the American Physical Society's annual meeting. This is considered to be the first academic talk about nanotechnology. ${ }^{5}$ He presented a lecture that was entitled "There's Plenty of Room at the Bottom". During this meeting, the following concept was presented: "why can't we write the entire 24 volumes of the Encyclopedia Britannica on the head of a pin?" The vision was to develop smaller machines, down to the molecular level. ${ }^{6,7}$ In this talk, Feynman explained that the laws of nature do not limit our ability to work at the atomic and molecular levels, but rather it is a lack of appropriate equipment and techniques that limit

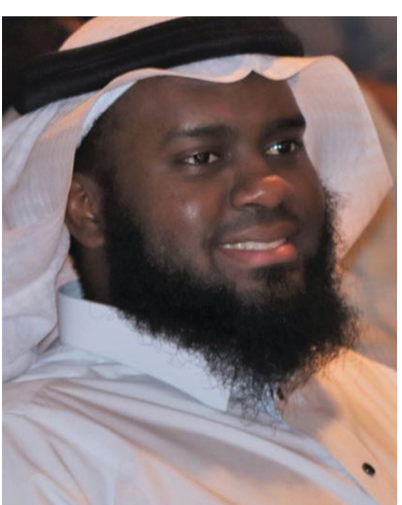

Wail Falath
Dr Wail Sulaiman Falath is the Director, Center for Environment \& Water of the Research Institute, and Assistant Professor at the Mechanical Engineering Department of King Fahd University of Petroleum \& Minerals. He is the Dean of Student Affairs, as well. Dr Falath has a PhD degree in Polymers, Textiles, and Fibers Engineering from Georgia Institute of Technology, USA. His PhD work was related to the fabrication of polymeric mixed matrix membranes for Reverse Osmosis water desalination. Dr Falath's research is focused on the environment, synthesis of membranes for water desalination, and surface modifications and characterization. this. ${ }^{8}$ Through this, the concept of modern technology was seeded. Due to this, he is often considered the father of modern nanotechnology. Norio Taniguchi might be the first person who used the term nanotechnology, in 1974. Norio Taniguchi stated: "nano-technology mainly consists of the processing of, separation, consolidation, and deformation of materials by one atom or one molecule." ${ }^{, 5}$ Before the 1980s, nanotechnology remained only an area for discussion, but the concept of nanotechnology was seeded in the minds of researchers with the potential for future development.

The invention of various spectroscopic techniques sped up research and innovations in the field of nanotechnology. IBM researchers developed scanning tunneling microscopy (STM) in 1982, and with STM it became feasible to attain images of single atoms on "flat" (i.e., not a tip) surfaces. ${ }^{10}$ Atomic force microscopy (AFM) was invented in 1986, and it has become the most crucial scanning probe microscope technique. ${ }^{11}$ The motivation to develop hard discs with high storage density stimulated the measurement of electrostatic and magnetic forces. This led to the development of Kelvin-probe-, electrostatic-, and magnetic-force microscopy. ${ }^{12}$ Currently, nanotechnology is rapidly evolving and becoming part of almost every field related to materials chemistry. The field of nanotechnology is evolving every day, and now powerful characterization and synthesis tools are available for producing nanomaterials with better-controlled dimensions.

Nanotechnology is an excellent example of an emerging technology, offering engineered nanomaterials with the great potential for producing products with substantially improved performances. $^{13}$ Currently, nanomaterials find commercial roles in scratch-free paints, surface coatings, electronics, cosmetics, environmental remediation, sports equipment, sensors, and energystorage devices. ${ }^{14}$ This review attempts to provide information in a single platform about the basic concepts, advances, and trends relating to nanomaterials via covering the related information and discussing synthesis methods, properties, and possible opportunities relating to the broad and fascinating area of nanomaterials (Scheme 1). It is not easy to cover all the literature related to nanomaterials, but important papers from history and the current literature are discussed in this review. This review provides fundamental insight for researchers, quickly capturing the advances in and properties of various classes of nanomaterials in one place.

\section{Descriptions of terms associated with nanomaterials}

Rigorous definitions of nanomaterials and associated terms are debatable and still not fixed. For this reason, huge room has been left around the interpretation and classification of nanomaterials. The definition of nanomaterials is not straightforward. In the literature, nanomaterials are perceived in different ways due to the absence of a rigorous definition. Many researchers have used the term nanomaterial if the size is a few nanometers or smaller than a few tens of nanometers, whereas others have even used the term nanomaterial for anything less than a micrometer. The physical and chemical properties of nanomaterials depend 


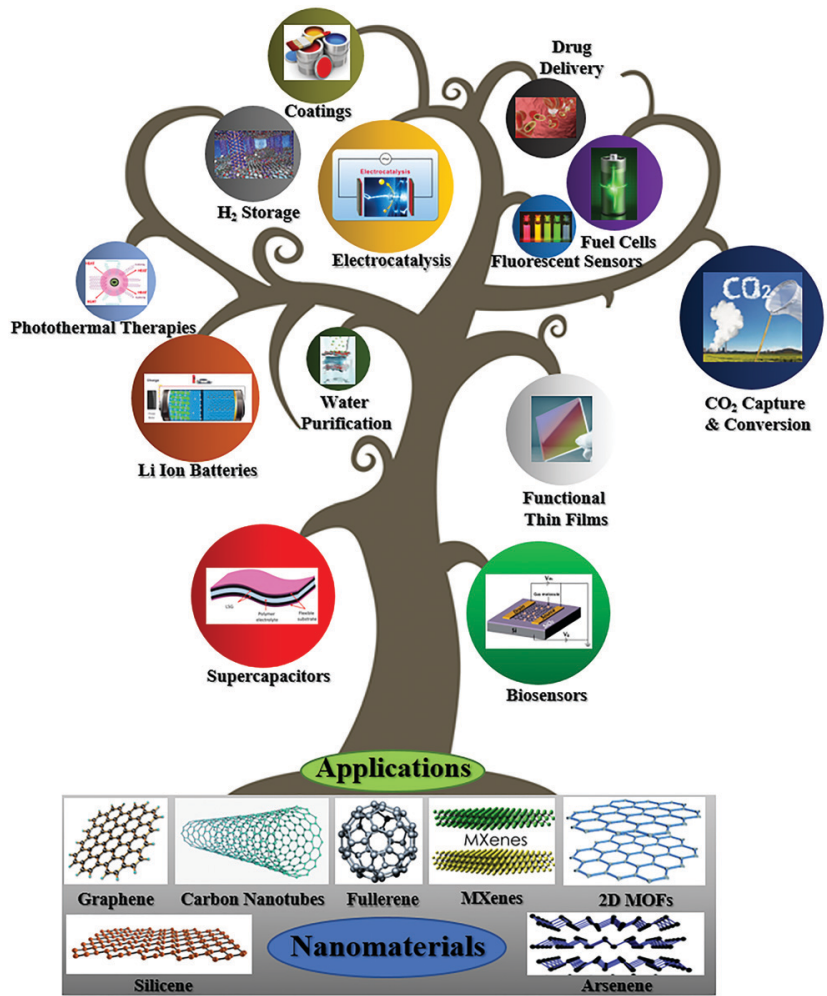

Scheme 1 A schematic representation of nanomaterials and their applications.

upon their precise composition, shape, and size. The effects of nanomaterial on health and the environment also depend upon their size, shape, etc. ${ }^{15} \mathrm{~A}$ single internationally accepted definition of nanomaterials is challenging to find, ${ }^{16}$ and a rigorous definition of nanomaterials is still under discussion in the scientific community. Definitions and descriptions of terms associated with nanomaterials are provided in Table 1 . However, these terms are not rigorous; instead, they provide a general perception collected from the available literature.

\section{Approaches for the synthesis of nanomaterials}

Two main approaches are used for the synthesis of nanomaterials (Fig. 1): top-down approaches and bottom-up approaches.

\subsection{Top-down approaches}

In top-down approaches, bulk materials are divided to produce nanostructured materials. Top-down methods include mechanical milling, laser ablation, etching, sputtering, and electro-explosion.

3.1.1. Mechanical milling. Mechanical milling is a costeffective method for producing materials at the nanoscale level from bulk materials. Mechanical milling is an effective method for producing blends of different phases, and it is helpful in the production of nanocomposites. The principle of the ball milling method is shown in Fig. $2 .{ }^{25}$ Mechanical milling is used to produce oxide- and carbide-strengthened aluminum alloys, wear-resistant spray coatings, aluminum/nickel/magnesium/copper-based nanoalloys, and many other nanocomposite materials. ${ }^{26}$ Ball-milled carbon nanomaterials are considered a novel class of nanomaterial,

Table 1 A description of various terms associated with nanomaterials

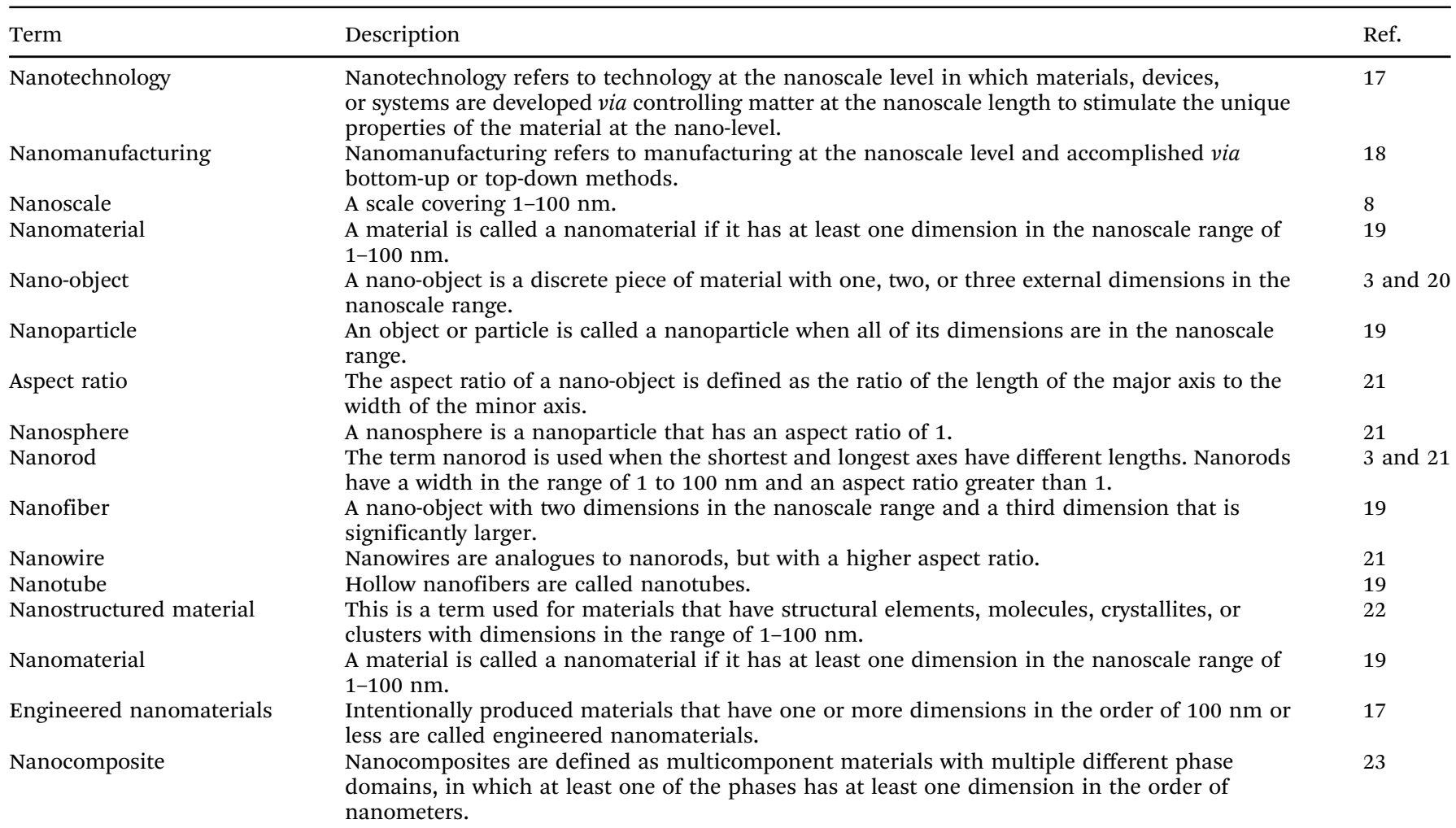




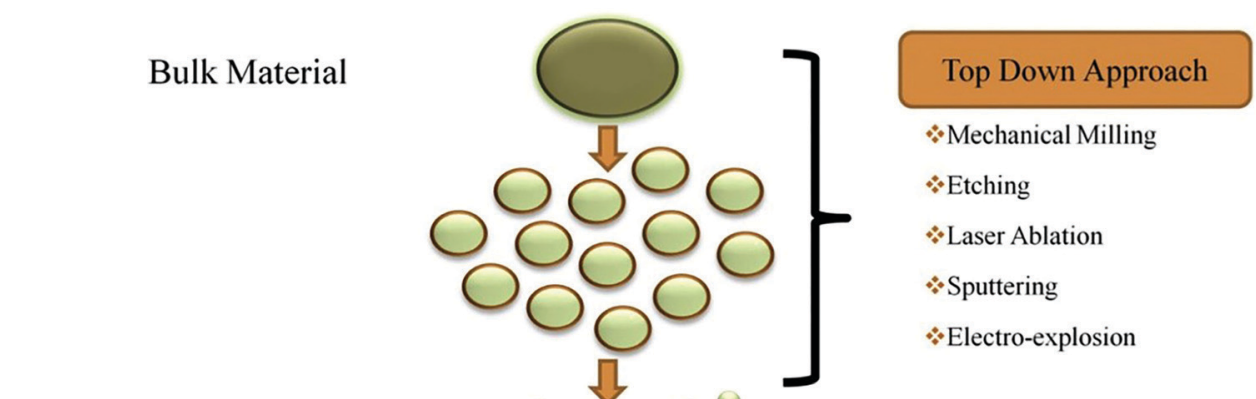

Nanoparticles

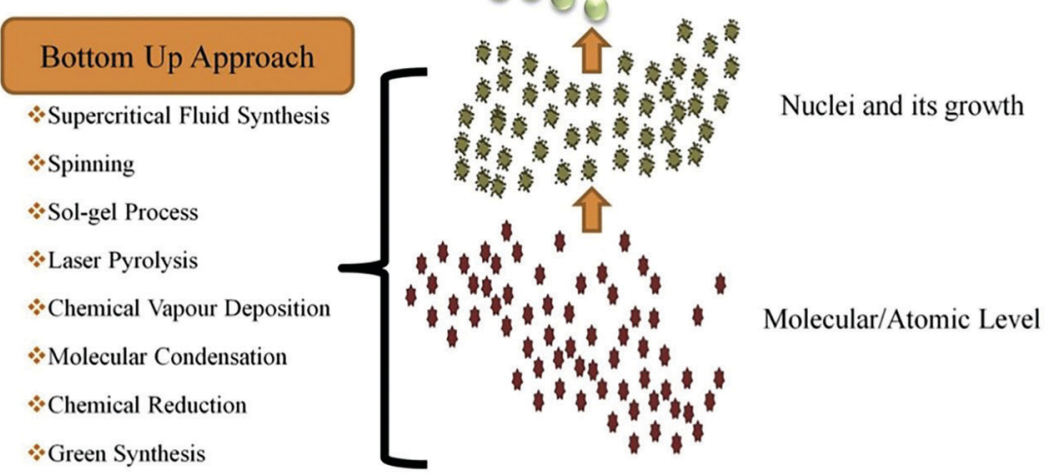

Fig. 1 The synthesis of nanomaterials via top-down and bottom-up approaches. Reprinted with permission from ref. 24. Copyright: (C)2019, Elsevier B.V. All rights reserved.
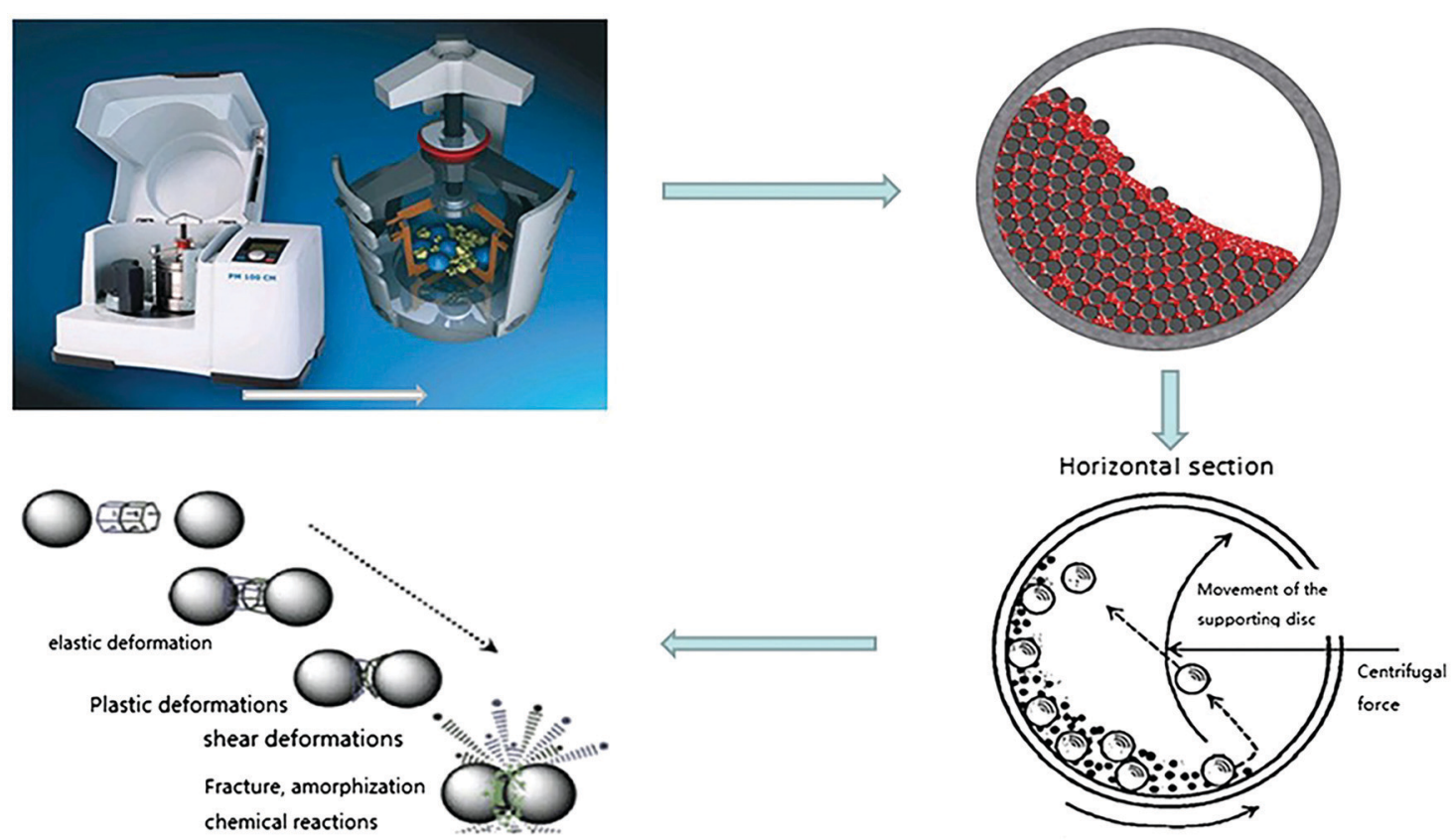

Rotation of the milling bowl

Fig. 2 The principle of the ball milling method. Reprinted with permission from ref. 25. Copyright: (C)2016, John Wiley \& Sons, Ltd.

providing the opportunity to satisfy environmental remediation, energy storage, and energy conversion demands. ${ }^{27}$
3.1.2. Electrospinning. Electrospinning is one of the simplest top-down methods for the development of nanostructured 

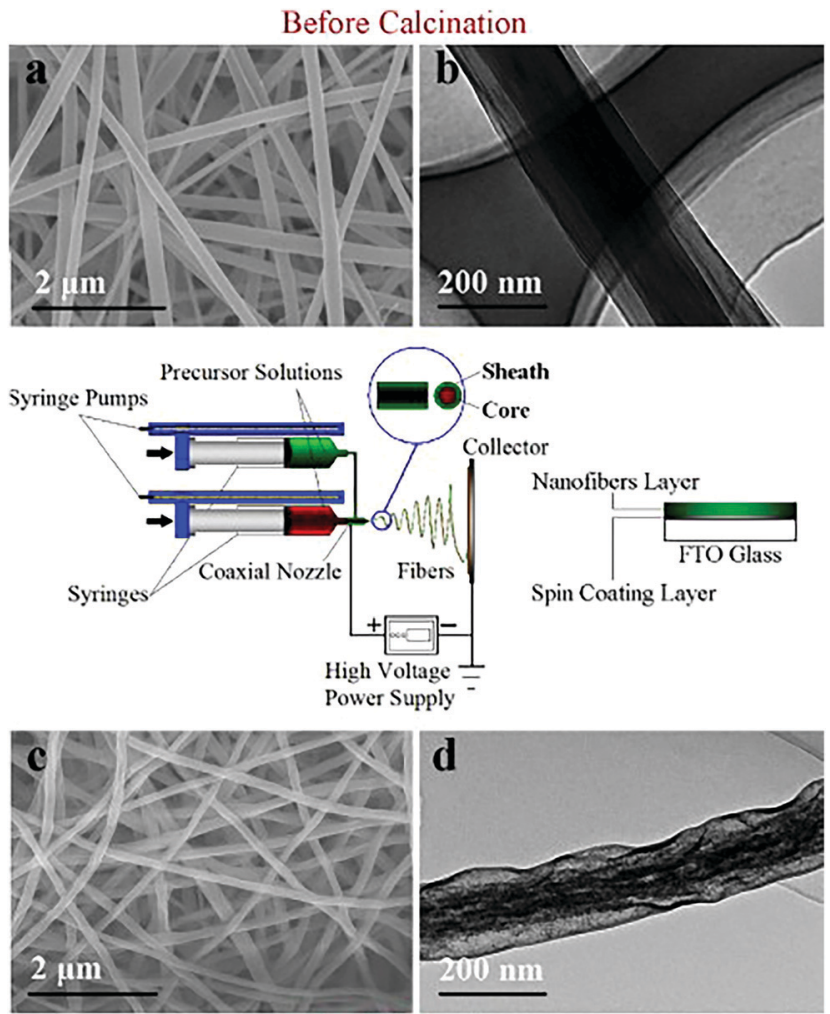

After Calcination

Fig. 3 A schematic diagram of the coaxial electrospinning technique (center), and FESEM ( $a$ and $c$ ) and TEM ( $b$ and $d$ ) images of fibers before and after calcination. Reprinted with permission from ref. 30. Copyright: (C)2012, Elsevier Ltd. All rights reserved.

materials. It is generally used to produce nanofibers from a wide variety of materials, typically polymers. ${ }^{28}$ One of the important breakthroughs in electrospinning was coaxial electrospinning. In coaxial electrospinning, the spinneret comprises two coaxial capillaries. In these capillaries, two viscous liquids, or a viscous liquid as the shell and a non-viscous liquid as the core, can be used to form core-shell nanoarchitectures in an electric field. Coaxial electrospinning is an effective and simple top-down approach for achieving core-shell ultrathin fibers on a large scale. The lengths of these ultrathin nanomaterials can be extended to several centimeters. This method has been used for the development of core-shell and hollow polymer, inorganic, organic, and hybrid materials. ${ }^{29}$ A schematic diagram of the coaxial electrospinning approach can be seen in Fig. $3 .^{30}$

3.1.3. Lithography. Lithography is a useful tool for developing nanoarchitectures using a focused beam of light or electrons. Lithography can be divided into two main types: masked lithography and maskless lithography. ${ }^{31}$ In masked nanolithography, nanopatterns are transferred over a large surface area using a specific mask or template. Masked lithography includes photolithography, ${ }^{32}$ nanoimprint lithography, ${ }^{33}$ and soft lithography. ${ }^{34}$ Maskless lithography includes scanning probe lithography, ${ }^{35}$ focused ion beam lithography, ${ }^{36}$ and electron beam lithography. In maskless lithography, arbitrary nanopattern writing is carried out without the involvement of a mask. 3D freeform micro-nano-fabrication can
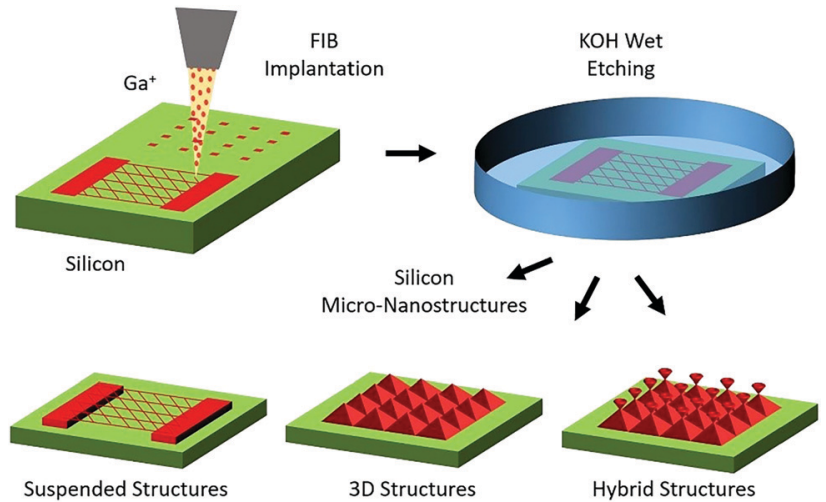

Fig. 4 A schematic diagram of the fabrication of 3D micro-nanostructures with an ion beam through bulk Si structuring. This involves implantation in Si through Ga FIB lithography and mask-writing at nanometer resolution, subsequent anisotropic wet etching in $\mathrm{KOH}$ solution, and the fabrication of Si micro-nanostructures via the selective removal of the unimplanted region. Reprinted with permission from ref. 37. Copyright: C) 2020, Elsevier B.V. All rights reserved.

be achieved via ion implantation with a focused ion beam in combination with wet chemical etching, as shown in Fig. $4 .^{37}$

3.1.4. Sputtering. Sputtering is a process used to produce nanomaterials via bombarding solid surfaces with high-energy particles such as plasma or gas. Sputtering is considered to be an effective method for producing thin films of nanomaterials. $^{38}$ In the sputtering deposition process, energetic gaseous ions bombard the target surface, causing the physical ejection of small atom clusters depending upon the incident gaseous-ion energy (Fig. 5). ${ }^{39,40}$ The sputtering process can be performed in different ways, such as utilizing magnetron, radio-frequency diode, and DC diode sputtering. ${ }^{40}$ In general, sputtering is performed in an evacuated chamber, to which the sputtering gas is introduced. A high voltage is applied to the cathode target and free electrons collide with the gas to produce gas ions. The positively charged ions strongly accelerate in the electric field towards the cathode target, which these ions continuously hit, resulting in the ejection of atoms from the surface of the target. ${ }^{41}$ Magnetron sputtering is used to produce WSe ${ }_{2}$-layered nanofilms on $\mathrm{SiO}_{2}$ and carbon paper substrates. ${ }^{42}$ The sputtering technique is interesting because the sputtered nanomaterial composition remains the same as the target material with fewer impurities, and it is cost-effective compared with electron-beam lithography. ${ }^{43}$

3.1.5. The arc discharge method. The arc discharge method is useful for the generation of various nanostructured materials. It is more known for producing carbon-based materials, such as fullerenes, carbon nanohorns (CNHs), carbon nanotubes, few-layer graphene (FLG), and amorphous spherical carbon nanoparticles. ${ }^{44}$ The arc discharge method has great significance in the generation of fullerene nanomaterials. In the formation process, two graphite rods are adjusted in a chamber in which a certain helium pressure is maintained. Filling the chamber with pure helium is important as the presence of moisture or oxygen inhibits fullerene formation. Carbon rod vaporization is driven by arc discharge between the ends of the graphite rods. ${ }^{45}$ 


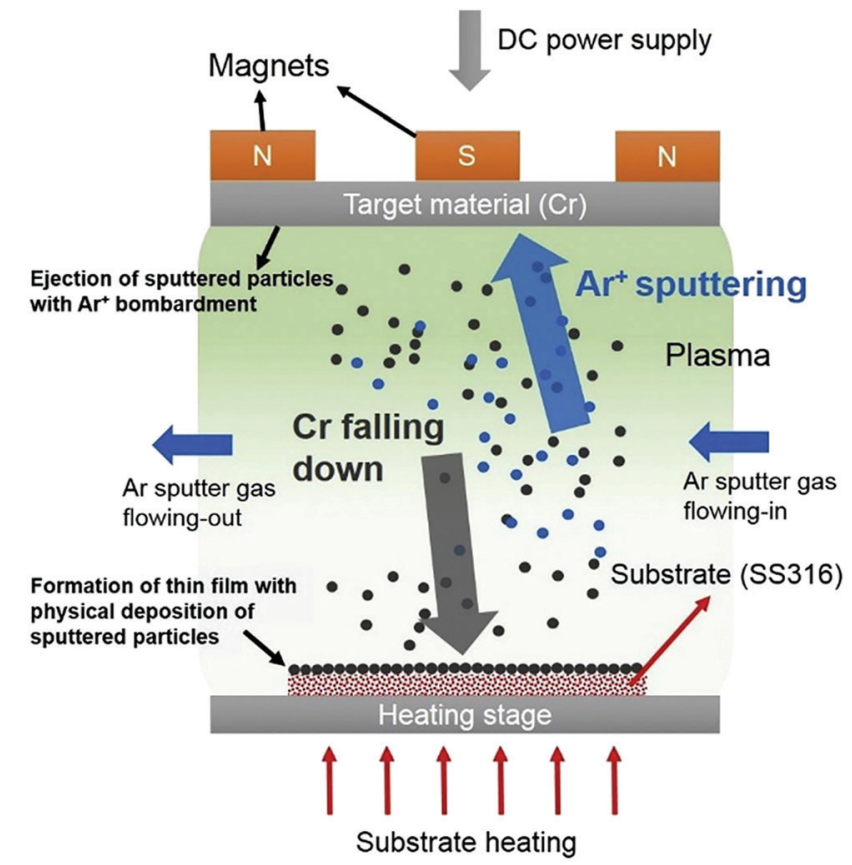

Fig. 5 A schematic diagram of the DC magnetron sputtering process. Reprinted with permission from ref. 39. Copyright: (C)2017, Elsevier Ltd. All rights reserved.

The conditions under which arc discharge takes place play a significant role in achieving new forms of nanomaterials. The conditions under which different carbon-based nanomaterials are formed via the arc discharge method are explained in Fig. 6. Various carbon-based nanomaterials are collected from different positions during the arc discharge method, as their growth mechanisms differ. ${ }^{44}$ MWCNTs, high-purity polyhedral graphite particles, pyrolytic graphite, and nano-graphite particles can be collected from either anode or cathode deposits or deposits at both electrodes. ${ }^{46-48}$ Apart from the electrodes, carbon-based nanomaterials can also be collected from the inner chamber. Different morphologies of single-wall carbon nanohorns (SWCNHs) can be obtained under different atmospheres. For example, 'dahlia-like'

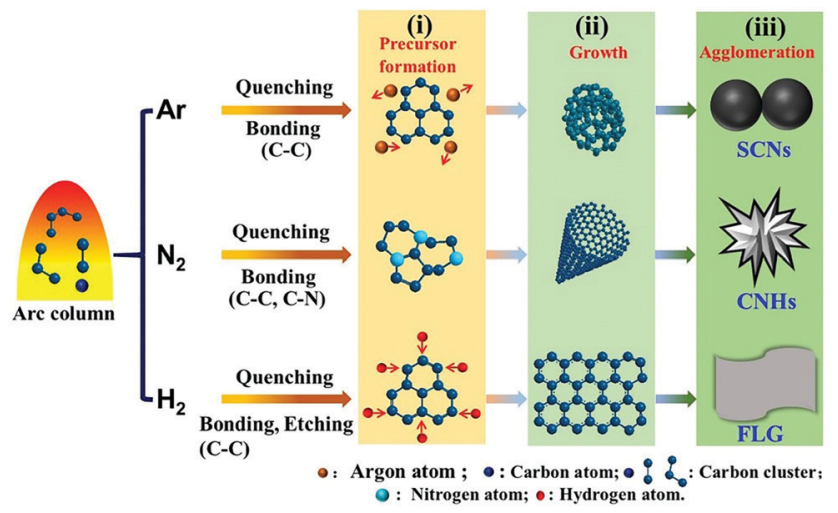

Fig. 6 A schematic illustration of the formation mechanisms of carbon nanomaterials on the inner wall of the chamber using different gases via a DC arc discharge approach. Reprinted with permission from ref. 44 Copyright: (C)2018, Elsevier Ltd. All rights reserved.
SWCNHs are produced under an ambient atmosphere, whereas 'bud-like' SWCNHs are generated under $\mathrm{CO}$ and $\mathrm{CO}_{2}$ atmospheres. ${ }^{49}$ The arc discharge method can be used to efficiently achieve graphene nanostructures. The conditions present during the synthesis of graphene can affect its properties. Graphene sheets prepared via a hydrogen arc discharge exfoliation method are found to be superior in terms of electrical conductivity and have good thermal stability compared to those obtained via argon arc discharge. ${ }^{50}$

3.1.6. Laser ablation. Laser ablation synthesis involves nanoparticle generation using a powerful laser beam that hits the target material. During the laser ablation process, the source material or precursor vaporizes due to the high energy of the laser irradiation, resulting in nanoparticle formation. Utilizing laser ablation for the generation of noble metal nanoparticles can be considered as a green technique, as there is no need for stabilizing agents or other chemicals. ${ }^{51}$ A wide range of nanomaterials can be produced through this technique, such as metal nanoparticles, ${ }^{52}$ carbon nanomaterials, ${ }^{53,54}$ oxide composites, ${ }^{55}$ and ceramics. ${ }^{56}$ Pulsed laser ablation in liquids is an exciting approach for producing monodisperse colloidal nanoparticle solutions without using surfactants or ligands. The nanoparticle properties, such as average size and distribution, can be tuned via adjusting the fluence, wavelength, and laser salt addition. It can be seen in Fig. 7 that the sizes of as-synthesized Pd nanoparticles are substantially affected by the wavelength and fluence of the pulsed laser. ${ }^{57}$

\subsection{Bottom-up approaches}

3.2.1. Chemical vapor deposition (CVD). Chemical vapor deposition methods have great significance in the generation of carbon-based nanomaterials. In CVD, a thin film is formed on the substrate surface via the chemical reaction of vapor-phase precursors. ${ }^{58}$ A precursor is considered suitable for CVD if it has adequate volatility, high chemical purity, good stability during evaporation, low cost, a non-hazardous nature, and a long shelf-life. Moreover, its decomposition should not result in residual impurities. ${ }^{58}$ For instance, in the generation of carbon nanotubes via CVD, a substrate is placed in an oven and heated to high temperatures. Subsequently, a carboncontaining (such as hydrocarbons) gas is slowly introduced to the system as a precursor. At high temperatures, the decomposition of the gas releases carbon atoms, which recombine to form carbon nanotubes on the substrate. ${ }^{59}$ However, the choice of catalyst plays a significant role in the morphology and type of nanomaterial obtained. In the CVD-based preparation of graphene, $\mathrm{Ni}$ and Co catalysts provide multilayer graphene, whereas a $\mathrm{Cu}$ catalyst provides monolayer graphene. ${ }^{60}$ Overall, CVD is an excellent method for producing high-quality nanomaterials, ${ }^{61}$ and it is well-known for the production of two-dimensional nanomaterials (Fig. 8). ${ }^{62}$

3.2.2. Solvothermal and hydrothermal methods. The hydrothermal process is one of the most well-known and extensively used methods used to produce nanostructured materials. ${ }^{63,64}$ In the hydrothermal method, nanostructured materials are attained through a heterogeneous reaction carried out in an aqueous 

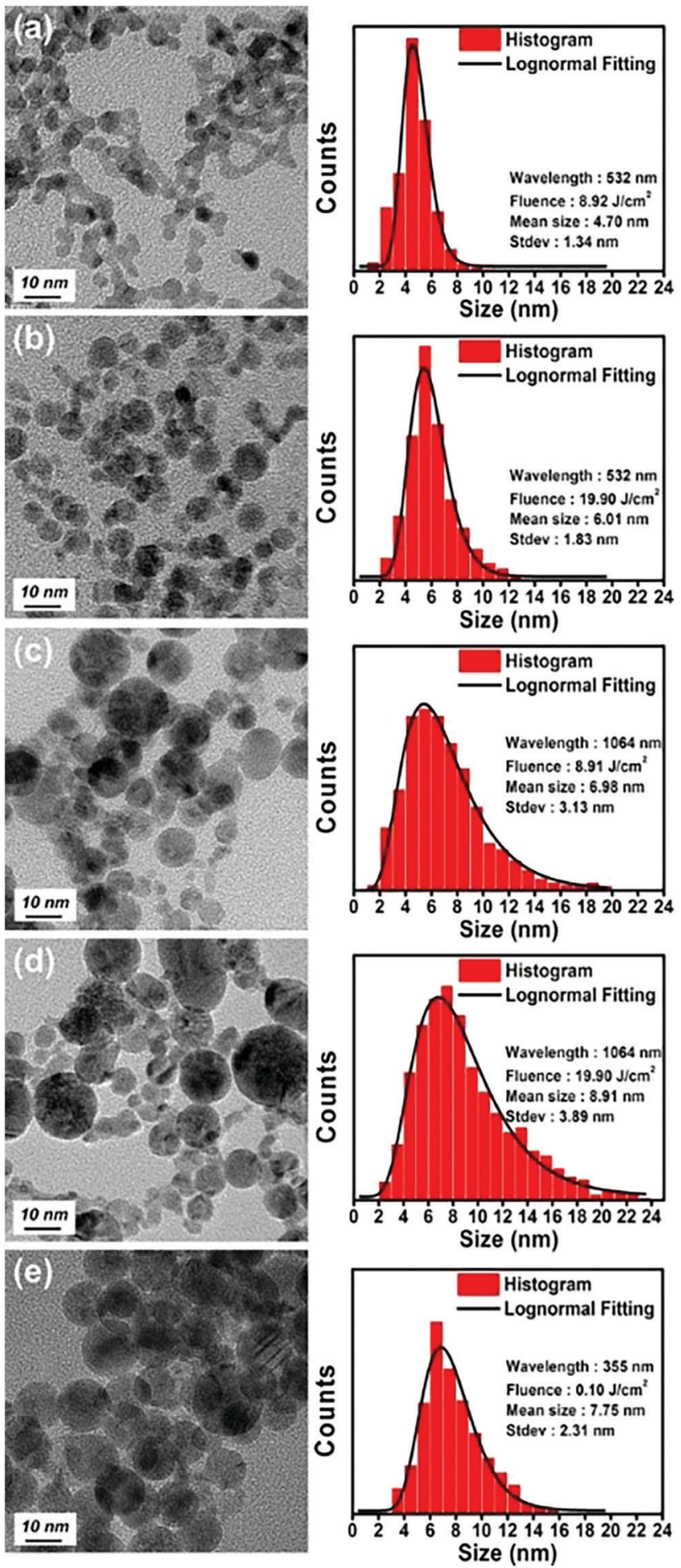

Fig. 7 TEM images, corresponding mean sizes, and standard deviations of palladium nanoparticles synthesized via laser ablation in water for $10 \mathrm{~min}$ at laser wavelengths and fluences of (a) $532 \mathrm{~nm}$ and $8.92 \mathrm{~J} \mathrm{~cm}^{-2}$, (b) $532 \mathrm{~nm}$ and $19.90 \mathrm{~J} \mathrm{~cm}^{-2}$, (c) $1064 \mathrm{~nm}$ and $8.92 \mathrm{~J} \mathrm{~cm}^{-2}$, (d) $1064 \mathrm{~nm}$ and $19.90 \mathrm{~J} \mathrm{~cm}^{-2}$, and (e) $355 \mathrm{~nm}$ and $0.10 \mathrm{~J} \mathrm{~cm}^{-2}$. Reprinted with permission from ref. 57. Copyright: (C)2017, Wiley-VCH Verlag GmbH \& Co. KGaA, Weinheim.

medium at high pressure and temperature around the critical point in a sealed vessel. ${ }^{65}$ The solvothermal method is like the hydrothermal method. The only difference is that it is carried out in a non-aqueous medium. Hydrothermal and solvothermal methods are generally carried out in closed systems. ${ }^{66}$ The microwave-assisted hydrothermal method has recently received significant attention for engineering nanomaterials, combining the merits of both hydrothermal and microwave methods. ${ }^{67}$ Hydrothermal and solvothermal methods are exciting and useful methods for producing various nano-geometries of materials, such as nanowires, nanorods, nanosheets, and nanospheres. ${ }^{68-70}$

3.2.3. The sol-gel method. The sol-gel method is a wetchemical technique that is extensively used for the development of nanomaterials. This method is used for the development of various kinds of high-quality metal-oxide-based nanomaterials. This method is called a sol-gel method as during the synthesis of the metal-oxide nanoparticles, the liquid precursor is transformed to a sol, and the sol is ultimately converted into a network structure that is called a gel. ${ }^{71}$ Conventional precursors for the generation of nanomaterials using the sol-gel method are metal alkoxides. The synthesis process of nanoparticles via the sol-gel method can be completed in several steps. In the first step, the hydrolysis of the metal oxide takes place in water or with the assistance of alcohol to form a sol. In the next step, condensation takes place, resulting in an increase in the solvent viscosity to form porous structures that are left to age. During the condensation or polycondensation process, hydroxo- (M-OH-M) or oxo- (M-O-M) bridges form, resulting in metal-hydroxo- or metal-oxo-polymer formation in solution. ${ }^{72}$ During the aging process, polycondensation continues, with changes to the structure, properties, and porosity. During aging, the porosity decreases, and the distance between the colloidal particles increases. After the aging process, drying takes place, in which water and organic solvents are removed from the gel. Lastly, calcination is performed to achieve nanoparticles. ${ }^{73}$ Fig. 9 shows film and powder formation using the sol-gel method. ${ }^{74}$ The factors that affect the final product obtained via the sol-gel method are the precursor nature, hydrolysis rate, aging time, $\mathrm{pH}$, and molar ratio between $\mathrm{H}_{2} \mathrm{O}$ and the precursor. ${ }^{75}$ The sol-gel method is economically friendly and has many other advantages, such as the produced material being homogeneous in nature, the processing temperature being low, and the method being a facile way to produce composites and complex nanostructures. ${ }^{73}$

3.2.4. Soft and hard templating methods. Soft and hard template methods are extensively used to produce nanoporous materials. The soft template method is a simple conventional method for the generation of nanostructured materials. The soft template method has been considered advantageous due to its straightforward implementation, relatively mild experimental conditions, and the development of materials with a range of morphologies. ${ }^{76}$ In the soft templating method, nanoporous materials are produced using plenty of soft templates, such as block copolymers, flexible organic molecules, and anionic, cationic, and non-ionic surfactants. ${ }^{77}$ Most prominent interactions between the soft templates and the precursors occur through hydrogen bonding, van der Waals forces, and electrostatic forces. ${ }^{78}$ Soft templates of 3D specifically arranged liquid crystalline micelles are used to synthesize $3 \mathrm{D}$ ordered mesoporous 
A

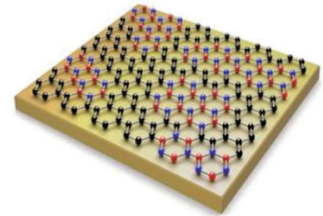

h-BNC growth by simultaneous in situ CVD on Cu

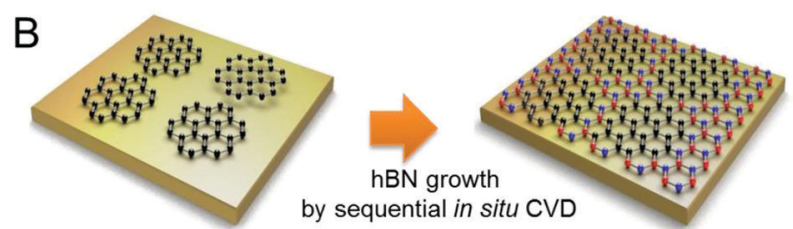

Graphene on Cu by CVD
Graphene-hBN

C
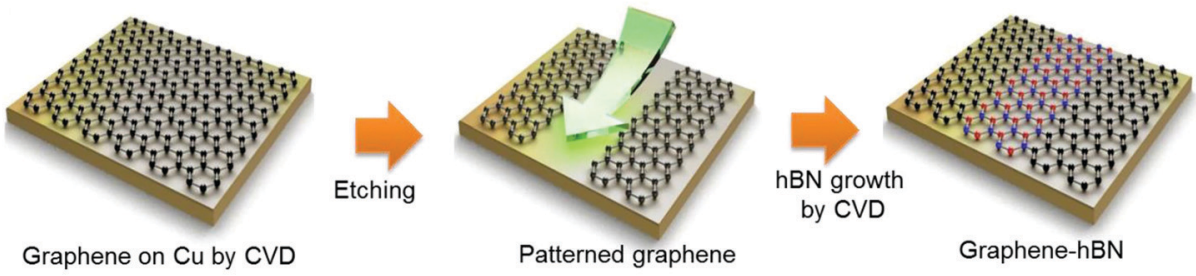

D
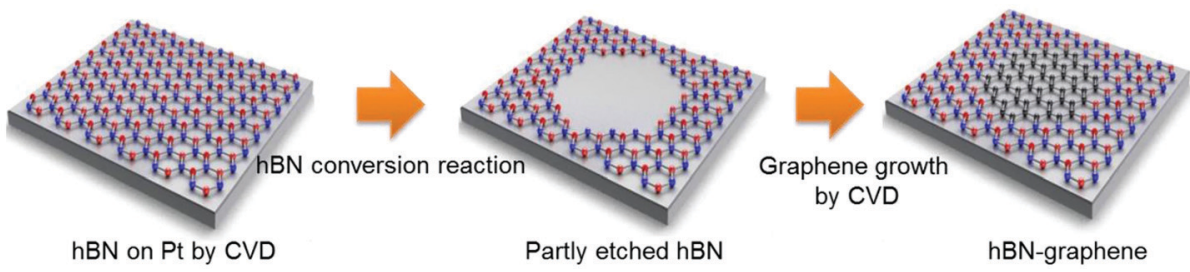

Fig. 8 A schematic diagram of the growth of in-plane graphene and hBN heterostructures via various techniques: (A) simultaneous in situ CVD growth, (B) sequential in situ CVD growth, (C) lithography-assisted growth, and (D) conversion growth. Reprinted with permission from ref. 62. Copyright: (C)2016, Elsevier B.V. All rights reserved.

structures. One of the classic examples involves mesoporous solids, such as lamellar (MCM-50), cubic (MCM-48), and hexagonal (MCM-41) ordered mesoporous silicas, being produced using alkyltrimethylammonium surfactant. ${ }^{79,80}$ Generally, for the synthesis of ordered mesoporous materials via a soft templating method, two processes called cooperative self-assembly and "true" liquid-crystal templating are adopted. ${ }^{77}$ Several factors can affect the mesoporous material structures derived from 3D arranged micelles, such as the surfactant and precursor concentrations, ratio of surfactant to precursor, surfactant structure, and environmental conditions. ${ }^{76}$ The nanoporous material pore sizes can be tuned via varying the surfactant carbon chain-length or introducing auxiliary pore-expanding agents. A range of nanostructured materials, such as mesoporous polymeric carbonaceous nanospheres, ${ }^{81}$ single crystal nanorods, ${ }^{82}$ porous aluminas, ${ }^{83}$ and mesoporous N-doped graphene, ${ }^{84}$ can be produced via the soft template method.

The hard template method is also called nano-casting. Welldesigned solid materials are used as templates, and the solid template pores are filled with precursor molecules to achieve nanostructures for required applications (Fig. 10). ${ }^{78}$ The selection of the hard template is critical for developing well-ordered mesoporous materials. It is desirable that such hard templates should maintain a mesoporous structure during the precursor conversion process, and they should be easily removable without disrupting the produced nanostructure. A range of materials has been used as hard templates, not limited to carbon black, silica, carbon nanotubes, particles, colloidal crystals, and wood shells. ${ }^{85}$ Three main steps are involved in the synthetic pathway for obtaining nanostructures via templating methods. In the first step, the appropriate original template is developed or selected. Then, a targeted precursor is filled into the template mesopores to convert them into an inorganic solid. In the final step, the original template is removed to achieve the mesoporous replica. ${ }^{86}$ Via using mesoporous templates, unique nanostructured materials such as nanowires, nanorods, 3D nanostructured materials, nanostructured metal oxides, and many other nanoparticles can be produced. ${ }^{87}$ From this brief discussion, it can be seen that a wide range of unique structured nanomaterials can be produced using soft and hard template methods.

3.2.5. Reverse micelle methods. The reverse micelle method is also a useful technique for producing nanomaterials with the desired shapes and sizes. An oil-in-water emulsion results in normal micelles, in which hydrophobic tails are aimed towards a core that has trapped oil droplets within it. However, reverse micelles are formed in the case of a water-in-oil emulsion, in which the hydrophilic heads are pointing towards a core that contains water. ${ }^{88}$ The core of the reverse micelles acts as a nanoreactor for the synthesis of nanoparticles. It acts as the water pool for developing nanomaterials. The size of these nanoreactors can be controlled by varying the water-to-surfactant ratio, ultimately affecting the size of the nanoparticles synthesized through this method. If the water concentration is decreased, this results in smaller water droplets, resulting in the formation of smaller nanoparticles. ${ }^{89}$ Thus, the reverse micelle method provides a facile route for synthesizing uniform nanoparticles with precisely 


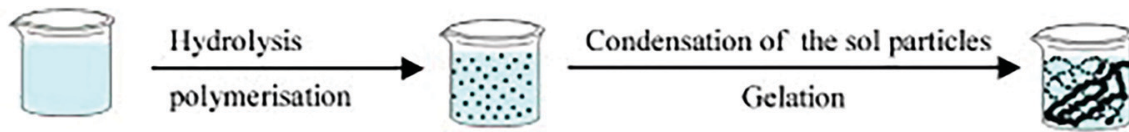

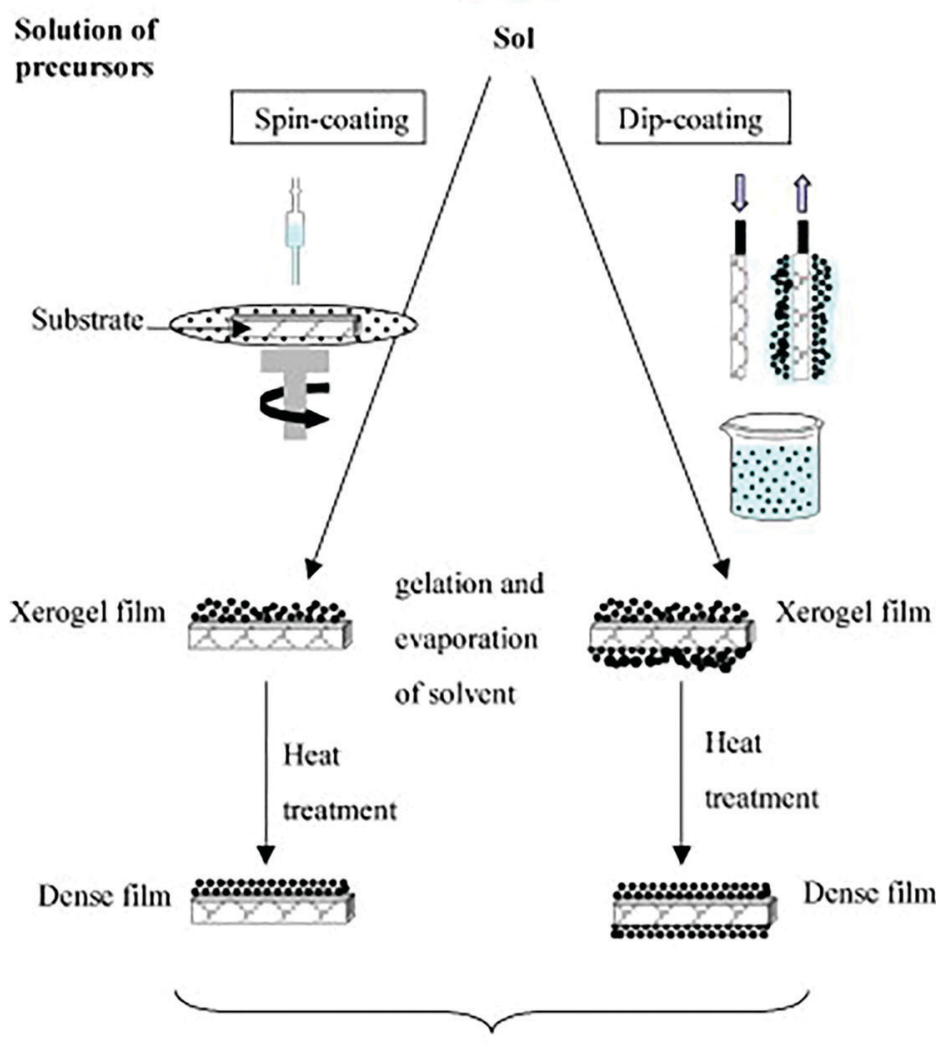

(a)

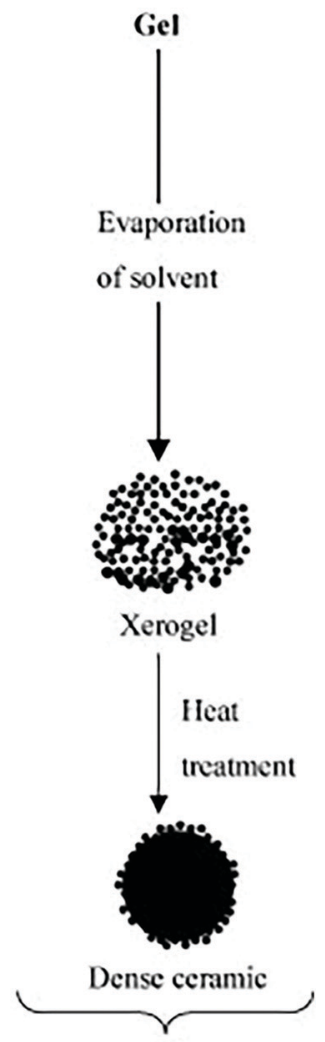

(b)

Fig. 9 An overview showing two sol-gel method synthesis examples: (a) films from a colloidal sol and (b) powder from a colloidal sol transformed into a gel. Reprinted with permission from ref. 74. Copyright: (C2010, Elsevier B.V. All rights reserved.

controlled size. Nanoparticles developed through the reverse micelle method are amazingly fine and monodispersed in nature. ${ }^{90}$ Fig. 11 demonstrates the synthesis of magnetic lipase-immobilized nanoparticles via the reverse micelle method. ${ }^{91}$

\section{Unique nanomaterial features}

The properties of matter at the nanoscale level are substantially distinct compared to bulk counterparts. Size-dependent effects become more prominent at the nanoscale. For example, $\mathrm{Au}$ solution appears yellow when in the bulk and it appears purple or red at the nanoscale level. The properties of nanomaterials can be tuned via tuning the nanomaterial size. ${ }^{92,93}$ At the nanoscale, the electronic properties are substantially changed compared to bulk materials. For example, boron in bulk form is not considered a metal, whereas a two-dimensional network of boron (borophene) appears to be an excellent 2D metal. ${ }^{94}$ Compared to their bulk counterparts, the mechanical properties of nanomaterials are considerably improved due to increases in crystal perfection or reductions in crystallographic defects. ${ }^{95}$

The electronic properties of semiconductors in the 1-10 nm range are controlled by quantum mechanical considerations.
Thus, nanospheres with diameters in the range of 1-10 $\mathrm{nm}$ are known as quantum dots. The optical properties of nanomaterials such as quantum dots strongly depend upon their shape and size. $^{96}$ A photogenerated electron-hole pair has an exciton diameter on the scale of 1-10 $\mathrm{nm}$. Thus, the absorption and emission of light by semiconductors could be controlled via tuning the nanoparticle size in this range. However, in the case of metals, the mean free path of electrons is $\sim 10-100 \mathrm{~nm}$ and, due to this, electronic and optical effects are expected to be observed in the range of $\sim 10-100 \mathrm{~nm}$. The colors of aqueous solutions of metal nanoparticles can be changed via changing the aspect ratio. Aqueous solutions of Ag NPs show different colors at different aspect ratios. A red shift in the absorption band appears with an increase in the aspect ratio (Fig. 12). ${ }^{21}$

Among a range of unique properties, the following key properties can be obtained upon tuning the sizes and morphologies of nanomaterials.

\subsection{Surface area}

The surface areas of nanomaterials are generally substantially high compared with their bulk counterparts, and this property is associated with all nanomaterials. ${ }^{97}$ 


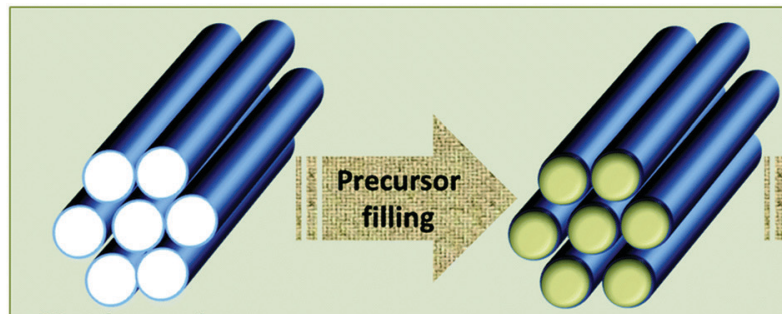

Hard template

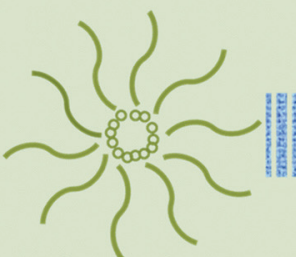

Soft template

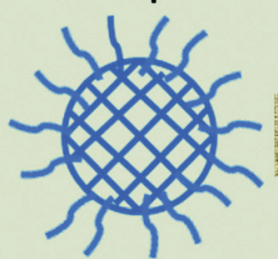

Colloidal template
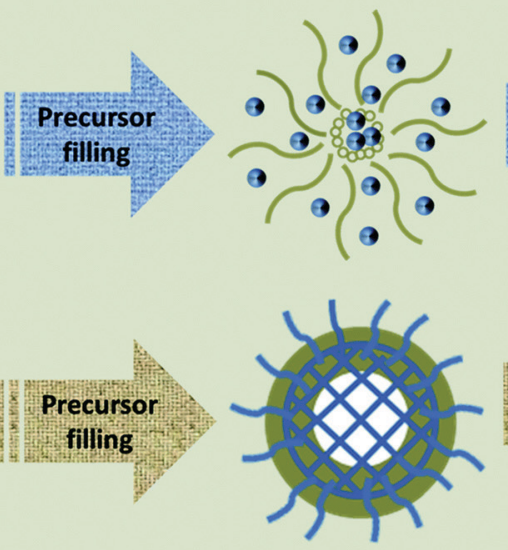
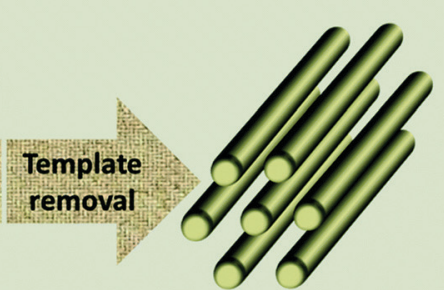

Final structure

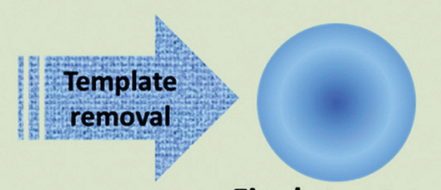

Final structure

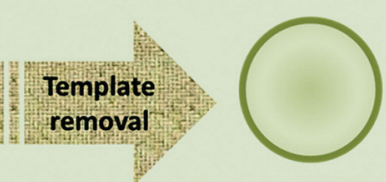

Final structure

Fig. 10 A schematic representation of the synthesis of materials using different types of templates. ${ }^{78}$ Published by The Royal Society of Chemistry.

\subsection{Magnetism}

The magnetic behavior of elements can change at the nanoscale. A non-magnetic element can become magnetic at the nanoscale level. ${ }^{93}$

\subsection{Quantum effects}

Quantum effects are more pronounced at the nanoscale level. However, the size at which these effects will appear strongly depends upon the nature of the semiconductor material. ${ }^{98}$

(a)

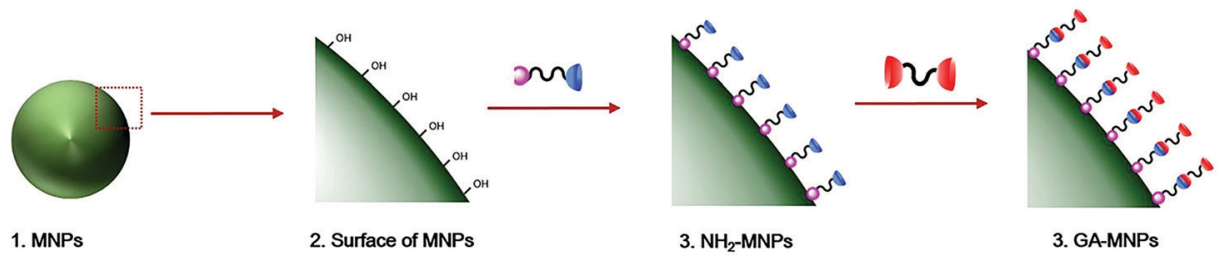

(b)

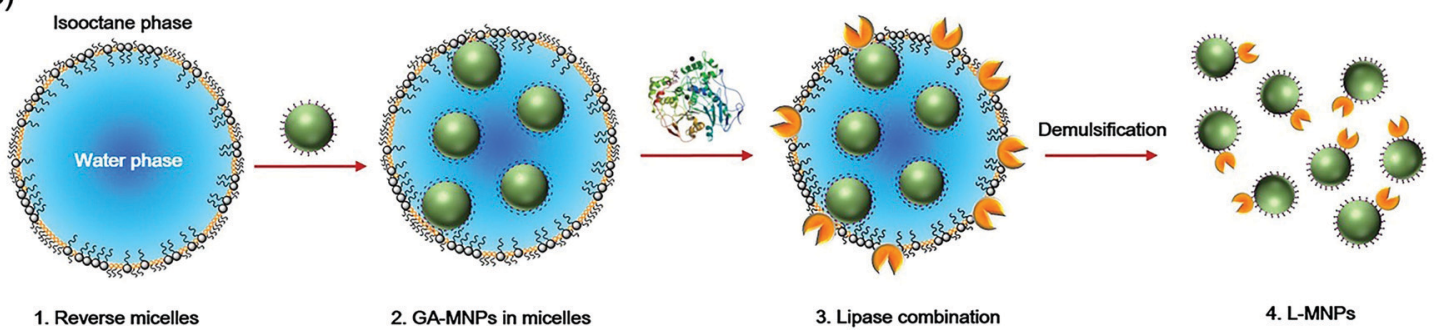

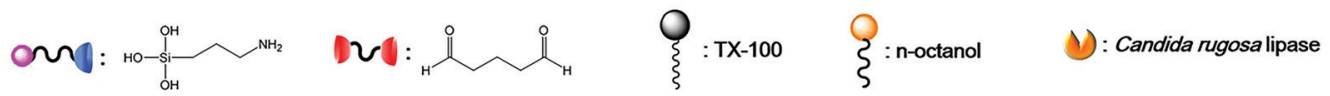

Fig. 11 (a) A schematic diagram showing the synthetic steps to GA-MNPs. (b) The synthesis of L-MNPs through a non-ionic reverse micelle method. ${ }^{91}$ 

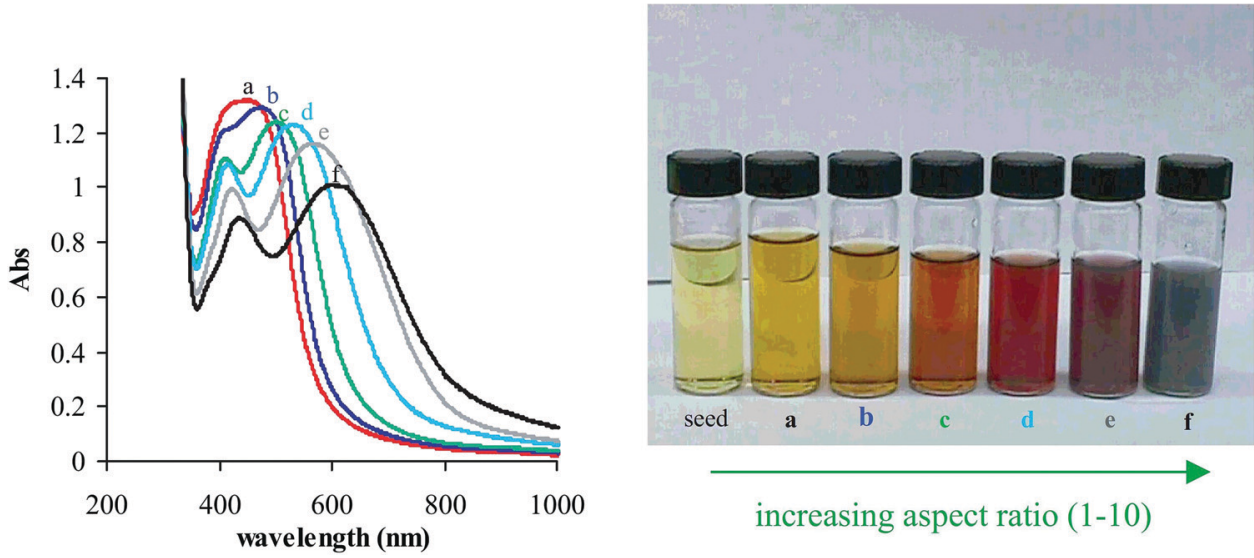

increasing aspect ratio (1-10)

Fig. 12 Aqueous solutions of silver nanoparticles show wide variations in visible color depending on the aspect ratio of the suspended nanoparticles. The far left of the photograph shows silver nanospheres (4 $\mathrm{nm}$ in diameter) that are used as seeds for subsequent reactions, while (a-f) show silver nanorods with increasing aspect ratios from 1-10. The corresponding visible absorption spectra for (a)-(f) are also shown in the left panel. Reprinted with permission from ref. 21. Copyright: (C)2002, WILEY-VCH Verlag GmbH, Weinheim, Fed. Rep. of Germany.

\subsection{High thermal and electrical conductivity}

According to the nature of the nanomaterial, extraordinary thermal and electrical conductivity can be exhibited at the nanoscale level compared to bulk counterparts. One example of this is graphene attained from graphite. ${ }^{99}$

\subsection{Excellent mechanical properties}

Nanomaterials exhibit excellent mechanical properties that are absent in their macroscopic counterparts. ${ }^{100}$

\subsection{Excellent support for catalysts}

2D sheets of various nanomaterials have provided the opportunity for the good dispersion of nanoparticles of active catalyst, enhancing the catalyst performance substantially. ${ }^{101}$ Recently, catalysts have been atomically dispersed on $2 \mathrm{D}$ sheets of nanomaterials to boost performance. $^{102}$

\subsection{Antimicrobial activity}

Some nanomaterials possess antiviral, antibacterial, and antifungal properties and have an excellent capacity to deal with pathogen-related diseases. ${ }^{103,104}$

Overall, these features have made nanoscale materials valuable for a wide range of applications, substantially boosting the performances of various devices and materials in a number of fields. Details of various nanomaterials, their properties, and applications in various fields will be discussed below.

\section{Nanomaterials, characteristics, and applications}

\subsection{Special carbon-based nanomaterials}

The carbon-based nanomaterial family consists of nanomaterials that have been extensively explored for various applications due to their outstanding features. The extraordinary properties of tunable carbon-based nanomaterials have attracted great interest for use in new technologies and addressing modern challenges. ${ }^{105,106}$ The carbon family consists of several unique nanomaterials, including CNTs, fullerenes, graphene, carbon nanohorns, carbonbased quantum dots, and many others. These nanomaterials are briefly discussed in this section, elaborating on their key characteristics and significance.

5.1.1. Fullerenes. Fullerenes are an amazing allotrope of carbon and they were discovered in 1985 . Fullerenes are highly symmetrical cages of $\mathrm{sp}^{2}$-hybridized carbon atoms. Fullerenes are distinct molecules, and they consist of a specific number of carbon atoms, making them different from the other allotropes of carbon. Fullerenes appear with different sizes according to the number of carbon atoms, such as $\mathrm{C}_{60}, \mathrm{C}_{70}, \mathrm{C}_{72}, \mathrm{C}_{76}, \mathrm{C}_{84}$, and $\mathrm{C}_{100 .}{ }^{107}$ Among them, the most abundant and famous example is $\mathrm{C}_{60}$ fullerene. $\mathrm{C}_{60}$ fullerene consists of a hollow structure with 12 pentagons and 20 hexagons made up from 60 carbon atoms that are linked to each other through covalent bonds that are $\mathrm{sp}^{2}$ hybridized in nature, and they show icosahedral symmetry. ${ }^{108}$ In fullerenes, the five-membered rings are isolated by six-membered rings. Fullerene $\left(\mathrm{C}_{60}\right)$ is considered an ideal zero-dimensional material due to its small, spherical, and isotropic nature. ${ }^{109}$ The sphere is considered to be one of the more stable structures in nature, and the same is true for fullerene.

In the carbon-based nanomaterial family, fullerenes were the first symmetric material, and they provided new perspectives in the nanomaterials field. This led to the discovery of other carbonbased nanostructured materials, such as carbon nanotubes and graphene. ${ }^{110}$ Fullerenes are present in nature and interstellar space. ${ }^{111}$ Interestingly, fullerenes were the molecule of the year in 1991 and attracted the most research projects compared to other scientific subjects during that period. ${ }^{112}$ Fullerenes possess several unique features that make them attractive for applications in different fields. Fullerenes display solubility to some extent in a range of solvents, and these characteristics make them unique compared to the other allotropes of carbon. ${ }^{108}$ 
The chemical modification of fullerenes is an exciting subject, improving their effectiveness for applications. There are two main ways to modify fullerenes: ${ }^{113}$ fullerene inner-space modification, and fullerene outer-surface modification.

Endohedral and exohedral doping examples are shown in Fig. $13 .{ }^{114}$ Fullerenes are hollow cages, and the interior acts as a robust nano-container for host target species when forming endohedral fullerene. ${ }^{115}$ Endohedral fullerenes do not always follow the isolated pentagon rule (IPR). ${ }^{116}$ To date, fullerene nanocages have received substantial consideration in the materials chemistry field due to their useful potential applications. Neutral and charged single atoms in free space are highly reactive and unstable. In the confined environment of fullerenes, these reactive species can be stabilized; for example, the $\mathrm{LaC}_{60}{ }^{+}$ion does not react with the $\mathrm{NH}_{3}, \mathrm{O}_{2}, \mathrm{H}_{2}$, or NO. Thus, reactive metals can be protected from the surrounding environment by trapping them inside fullerene cages. ${ }^{117}$ Another emerging carbon nanomaterial is endohedral fullerene containing lithium $\left(\mathrm{Li} @ \mathrm{C}_{60}\right) \cdot{ }^{118}$ Lithium metal is very reactive, and extreme controlled environmental conditions are required to preserve or use it. In other words, secure structures are required for lithium storage. Li-Based endohedral fullerene shows unique solid-state properties. The encapsulation of lithium atoms in fullerene helps to protect lithium atoms from external agents. Li-Based endohedral fullerenes have the potential to open the door to nanoscale lithium batteries. ${ }^{119}$ For the development of endohedral metallofullerenes, larger fullerenes are generally required, as they possess large cages to accommodate lanthanide and transition metal atoms more smoothly. ${ }^{118}$ Fullerene nanocages are useful for the storage of gases. Fullerene is under consideration for hydrogen storage. ${ }^{120,121}$

Exohedral fullerenes carry more potential for applications as outer surfaces can be more easily modified or functionalized. The exohedral doping of metals into fullerenes strongly affects the electronic properties via shifting electrons from the metal to the fullerene nanocage. ${ }^{122}$ The practical application of fullerenes can be achieved with tailor-made fullerene derivatives via chemical functionalization. As fullerene chemistry has matured, a wide range of functionalized fullerenes has been realized through simple synthetic routes. ${ }^{123}$ The combination of hydrogen-bonding motifs and fullerenes has allowed the modulation of 1D, 2D, and 3D fullerene-based architectures. ${ }^{124}$ The excellent electron affinities of fullerenes have shown great potential for eliminating reactive oxygen species. The presence of excess reactive oxygen

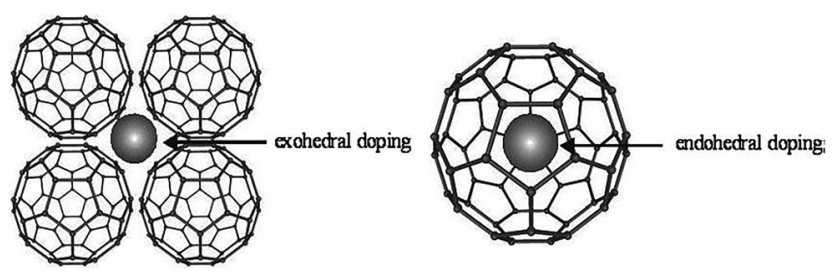

Fig. 13 A schematic representation of the two interstitial doping sites in $\mathrm{C}_{60}$, leading to exohedral and endohedral doping. Reprinted with permission from ref. 114. Copyright: (C2009, WILEY-VCH Verlag GmbH \& Co. KGaA, Weinheim. species can cause biological dysfunction or other health issues. The surfaces of fullerenes have been functionalized via musselinspired chemistry and Michael addition reactions for the fabrication of $\mathrm{C}_{60}-\mathrm{PDA}-\mathrm{GSH}$. The developed $\mathrm{C}_{60}-\mathrm{PDA}-\mathrm{GSH}$ nanoparticles demonstrated excellent potential for scavenging reactive oxygen species. ${ }^{125}$

Amphiphiles have great importance in industrial processes and daily life applications. Amphiphilic molecules consist of hydrophilic and hydrophobic parts, and they perform functions in water via forming two- and three-dimensional assemblies. Recently, conical fullerene amphiphiles ${ }^{126}$ have emerged as a new class of amphiphiles, in which a nonpolar apex is supplied by fullerenes and a hydrophilic part is achieved through functionalization. The selective functionalization of the fullerene on one side helps to achieve a supramolecule due to unique interfacial behavior. The unique supramolecular structure formed via the spontaneous assembly of one-sided selectively functionalized fullerenes through strong hydrophobic interactions between the fullerene apexes and polar functionalized portions is soluble in water. Conical fullerene amphiphiles are mechanically robust. Via upholding the structural integrity, conical fullerene amphiphiles can be readily aggregated with nanomaterials and biomolecules to form multicomponent agglomerates with controllable structural features. ${ }^{127}$ Fullerenes, after suitable surface modification, have excellent potential for use in drug delivery, but there have only been limited explorations of their drug delivery applications. ${ }^{128,129}$ Fullerene-based nano-vesicles have been developed for the delayed release of drugs. ${ }^{130}$ Water-soluble proteins have great potential in the field of nanomedicine. The water-soluble cationic fullerene, tetra(piperazino)[60] fullerene epoxide (TPFE), has been used for the targeted delivery of DNA and siRNA specifically to the lungs. ${ }^{131}$ For diseases in lungs or any other organ, efficient treatment requires the targeted delivery of active agents to a targeted place in the organ. The accumulation of micrometersized carriers in the lung makes lung-selective delivery difficult, as this may induce embolization and inflammation in the lungs. Size-controlled blood vessel carrier vehicles have been developed using tetra(piperazino)fullerene epoxide (TPFE). TPFE and siRNA agglutinate in the bloodstream with plasma proteins and, as a result, micrometer-sized particles are formed. These particles clog the lung capillaries and release siRNA into lungs cells; after siRNA delivery, they are immediately cleared from the lungs (Fig. 14). ${ }^{132}$

The supramolecular organization of fullerene $\left(\mathrm{C}_{60}\right)$ is a unique approach for producing shape-controlled moieties on the nano-, micro-, and macro-scale. Nano-, micro-, and macro-scale supramolecular assemblies can be controlled via manipulating the preparation conditions to achieve unique optoelectronic properties. ${ }^{133}$ The development of well-ordered and organized 1D, $2 \mathrm{D}$, and $3 \mathrm{D}$ fullerene assemblies is essential for achieving advanced optical and organic-based electronic devices. ${ }^{134}$ Fullerene-based nanostructured materials with new dimensions are being developed from zero-dimensional fullerene and tuned to achieve the desired characteristics. $1 \mathrm{D} \mathrm{C}_{60}$ fullerene nanowires have received substantial attention over other crystalline forms 
Preparation (1) Formation of TPFE-siRNA complex

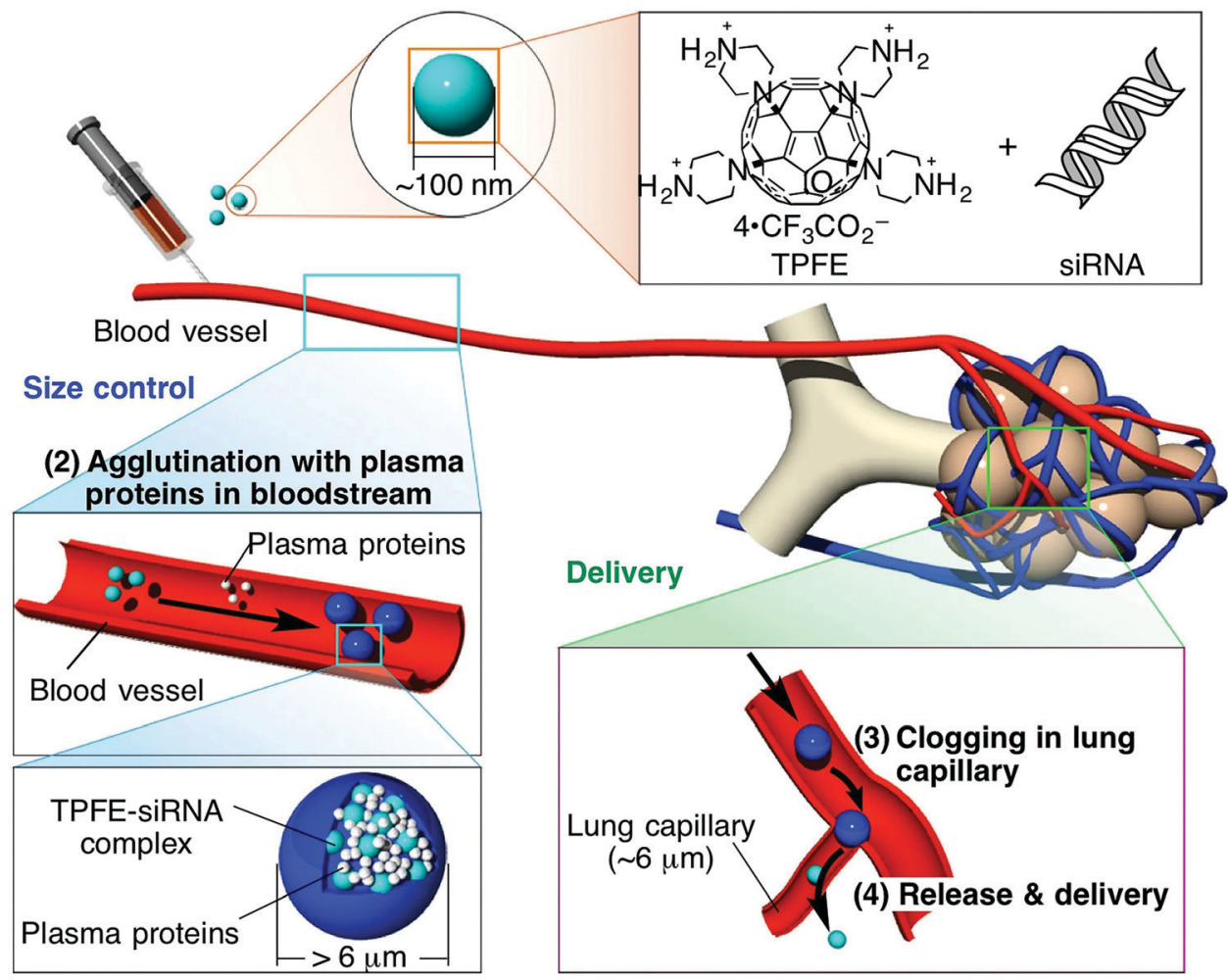

Fig. 14 The mechanism of the lung-specific delivery of siRNA mediated by tetra(piperazino)fullerene (TPFE): (1) TPFE aggregates with siRNA to form ca. $100 \mathrm{~nm}$-sized TPFE-siRNA complexes (light blue particles) via electrostatic interactions; (2) the complexes agglutinate with plasma proteins (white particles) in the bloodstream to form $>6 \mu \mathrm{m}$ particles (blue particles); (3) the particles clog and accumulate in the narrow lung capillaries; (4) the TPFEsiRNA complexes were delivered into lung cells and siRNA was released into lung cells. Reprinted with permission from ref. 132. Copyright: (C)2014, Springer Nature.

due to their excellent features of potential quantum confinement effects, low dimensionality, and large surface areas. ${ }^{135}$

Carbon nanomaterials are also used as supports for catalysts, and the main reasons to use them are their high surface areas and electrical conductivities. Carbon supports strongly influence the properties of metal nanoparticles. In fuel cells, the carbon support strongly affects the stability, electronic conductivity, mass transport properties, and electroactive surface area of the supported catalyst. ${ }^{136}$ In fuel cells, the degradation of some catalysts, such as platinum-based examples, and carbon is correlated and reinforced as a result of both being present. Carbon support oxidation is catalyzed by platinum and the oxidation of carbon accelerates platinum-catalyst release. Overall, this results in a loss of catalytically active surface area. ${ }^{137}$ Fullerenes are considered suitable support materials due to their excellent electrochemical activities and stability during electrochemical reactions. ${ }^{138}$ Due to their high stability and good conductivity, fullerenes can replace conventional carbon as catalyst support materials. Fullerenes are also used for the development of efficient solar cells. ${ }^{139}$

Apart from the applications mentioned above, fullerenes have a broader spectrum of applications where they can be used to improve outcomes considerably. Fullerenes have the potential to be used in the development of superconductors. ${ }^{140}$ The strong covalent bonds in fullerenes make them useful nanomaterials for improving the mechanical properties of composites. ${ }^{141}$ The combination of fullerenes with polymers can result in good flame-retardant and thermal properties. ${ }^{142}$ Fullerenes and their derivatives are used for the development of advanced lubricants. They are used as modifiers for greases and individual solid lubricants. ${ }^{138}$ Fullerenes have tremendous medicinal importance due to their anticancer, antioxidant, anti-bacterial, and anti-viral activities. ${ }^{104}$

Fullerenes are vital members of the carbon-based nanomaterial family and they certainly possess exceptional properties. This discussion further emphasizes their importance for advanced applications. However, the discovery of other carbon-based nanomaterials has put fullerenes in the shade, and the pace of their exploration has been reduced. As fullerenes are highly symmetrical molecules with unique properties, they can act as performance boosters, but more attention is needed from researchers for their practical expansion. ${ }^{110}$

5.1.2. CNTs. Carbon nanotubes are an essential member of the carbon nanomaterial family, and they entered the carbon family in 1991 after being discovered by S. Iijima. ${ }^{143}$ After the discovery of CNTs, extensive research was carried out to explore their properties for various applications. Later on, S. Iijima and Toshinari Ichihashi reported single-shell carbon nanotubes with a diameter of $1 \mathrm{~nm} \cdot{ }^{144}$ Carbon nanotubes are rolled sheets 
of single-layered $\mathrm{sp}^{2}$-hybridized carbon atoms. The surfaces of the nanotubes consist of $\mathrm{sp}^{2}$-hybridized carbon atoms that are arranged in hexagons. ${ }^{145}$ The carbon nanotubes can be further divided according to the number of rolled graphene sheets into single-walled carbon nanotubes, double-walled carbon nanotubes, and multi-walled carbon nanotubes.

Single-walled carbon nanotubes consist of a seamless oneatom-thick graphitic layer, in which carbon atoms are connected through strong covalent bonds. ${ }^{146}$ Double-walled carbon nanotubes consist of two single-walled carbon nanotubes. One carbon nanotube is nested in another nanotube to construct a doublewalled carbon nanotube. ${ }^{147}$ In multi-walled carbon nanotubes, multiple sheets of single-layer carbon atom are rolled up. In other words, many single-walled carbon nanotubes are nested within each other. From different types of nanotubes, it can be concluded that the nanotubes may consist of one, tens, or hundreds of concentric carbon shells, and these shells are separated from each other with a distance of $\sim 0.34 \mathrm{~nm} .{ }^{148}$ Carbon nanotubes can be synthesized via chemical vapor deposition, ${ }^{149}$ laser ablation, ${ }^{150}$ arc-discharge ${ }^{143}$ and gas-phase catalytic growth. ${ }^{151}$

Single-walled carbon nanotubes display a diameter of 0.4 to $2 \mathrm{~nm}$. The inner wall distance between double-walled carbon nanotubes was found to be in the range of 0.33 to $0.42 \mathrm{~nm}$. MWCNT diameters are usually in the range of 2-100 nm, and the inner wall distance is about $0.34 \mathrm{~nm} .{ }^{147,152}$ However, it is essential to note that the diameters and lengths of carbon nanotubes are not well defined, and they depend on the synthesis route and many other factors. The electrical conductivities of SWCNTs and MWCNTs are about $10^{2}-10^{6} \mathrm{~S} \mathrm{~cm}^{-1}$ and $10^{3}-10^{5} \mathrm{~S} \mathrm{~cm}^{-1}$, respectively. SWCNTs and MWCNTs also display excellent thermal conductivities of $\sim 6000 \mathrm{~W} \mathrm{~m}^{-1} \mathrm{~K}^{-1}$ and $\sim 2000 \mathrm{~W} \mathrm{~m}^{-1} \mathrm{~K}^{-1}$, respectively. CNTs remain stable in air at temperatures higher than $600{ }^{\circ} \mathrm{C} .{ }^{153}$ These properties indicate that CNTs have obvious advantages over graphite.

Single-walled carbon nanotubes can display metallic or semiconducting behavior. Whether carbon nanotubes show metallic or semiconducting behavior depends on the diameter and helicity of the graphitic rings. ${ }^{154}$ The rolling of graphene sheets leads to three different types of CNTs: chiral, armchair, and zigzag (Fig. 15). ${ }^{155}$

Carbon nanotubes demonstrate some amazing characteristics that make them valuable nanomaterials for possible practical applications. Theoretical and experimental studies of carbon nanotubes have revealed their extraordinary tensile properties. J. R. Xiao et al. used an analytical molecular structural mechanics model to predict SWCNT tensile strengths of 94.5 (zigzag nanotubes) and 126.2 (armchair nanotubes) GPa. ${ }^{156}$ In another study, the Young's modulus and average tensile strength of millimeterlong multi-walled carbon nanotubes were analyzed and found to be $34.65 \mathrm{GPa}$ and $0.85 \mathrm{GPa}$, respectively. ${ }^{157}$ Carbon nanotubes possess a high aspect ratio. Due to their high tensile strength, carbon nanotubes are used to enhance the mechanical properties of composites.

Carbon nanotubes have become an important industrial material and hundreds of tonnes are produced for applications. ${ }^{158}$ Their high

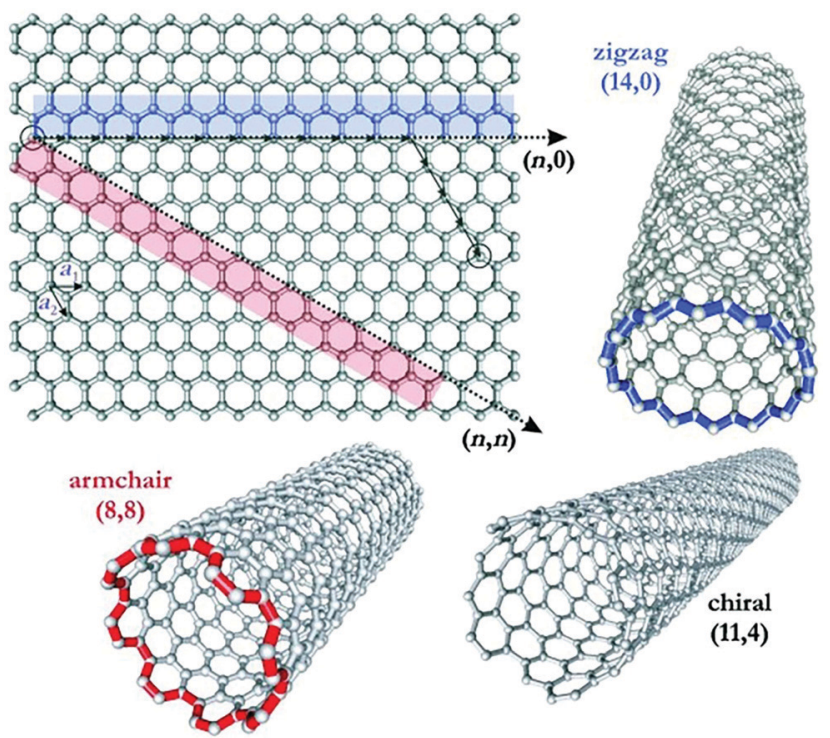

Fig. 15 The rolling up of a graphene sheet leading to the three different types of CNT. Reprinted with permission from ref. 155. Copyright: (C2005, WILEY-VCH Verlag GmbH \& Co. KGaA, Weinheim.

tensile strength and high aspect ratio have made carbon nanotubes an ideal reinforcing agent. ${ }^{159}$ Carbon nanotubes are lightweight in nature and are used to produce lightweight and biodegradable nanocomposite foams. ${ }^{160}$ The structural parameters of carbon nanotubes define whether they will be semiconducting or metallic in nature. This property of carbon nanotubes is considered to be effective for their use as a central element in the design of electronic devices such as rectifying diodes, ${ }^{161}$ single-electron transistors, ${ }^{162}$ and field-effect transistors. ${ }^{163}$ The chemical stability, nano-size, high electrical conductivity, and amazing structural perfection of carbon nanotubes make them suitable for electron field emitter applications. ${ }^{164}$ The unique set of mechanical and electrochemical properties make CNTs a valuable smart candidate for use in lithium-ion batteries. ${ }^{165}$ CNTs have the full potential to be used as a binderless free-standing electrode for active lithium-ion storage. CNT-based anodes can have reversible lithium-ion capacities exceeding $1000 \mathrm{~mA} \mathrm{~h} \mathrm{~g}^{-1}$, and this is a substantial improvement compared with conventional graphite anodes. In short, the following factors play a role in controlling and optimizing the performances of CNT-based composites: ${ }^{166}$ (i) the volume fraction of carbon nanotubes; (ii) the CNT orientation; (iii) the CNT matrix adhesion; (iv) the CNT aspect ratio; and (iv) the composite homogeneity.

For some applications, a proper stable aqueous dispersion of CNTs at a high concentration is pivotal to allow the system to perform its function efficiently and effectively. ${ }^{167}$ One of the major issues associated with carbon nanotubes is their poor dispersion in aqueous media due to their hydrophobic nature. Clusters of CNTs are formed due to van der Waals attraction, $\pi-\pi$ stacking, and hydrophobicity. The CNT clusters, due to their strong interactions, hinder solubility or dispersion in water or even organicsolvent-based systems. ${ }^{168}$ This challenging dispersion associated 
with CNTs has limited their use for promising applications, such as in biomedical devices, drug delivery, cell biology, and drug delivery. ${ }^{167}$ Carbon nanotube applications and inherent characteristics can be further tuned via suitable functionalization. The functionalization of carbon nanotubes helps scientists to manipulate the properties of carbon nanotubes and, without functionalization, some properties are not attainable. ${ }^{169}$ The functionalization of nanotubes can be divided into two main categories: covalent functionalization and non-covalent functionalization.

5.1.1. The covalent functionalization of CNTs. The covalent functionalization of CNTs has substantially improved the utility of nanomaterials for various applications. In covalent functionalization, the functional group is covalently attached to the sidewalls of the carbon nanotubes. Covalent functionalization is generally carried out via two approaches: (1) the activation of the carbon nanotubes by generating reactive species such as hydroxyl groups, amine groups, and carboxylic groups; and (2) the direct covalent attachment of the desired functionalities using radical addition, cycloaddition, and electrophilic and nucleophilic addition reactions. ${ }^{170}$ Functionalization via the sidewalls and ends/defects are two subcategories of the covalent functionalization of CNTs. ${ }^{171}$

The heating of CNTs under strongly acidic and oxidative conditions results in the formation of oxygen-containing functionalities. These functional groups, such as carboxylic acid, react further with other functional groups, such as amines or alcohols, to produce amide or ester linkages on the carbon nanotubes. ${ }^{172}$ One of the main issues preventing the utilization of CNTs for biomedical applications is their toxicity. The cytotoxicity of pristine carbon nanotubes can be reduced via introducing carbonyl, $-\mathrm{COOH}$, and $-\mathrm{OH}$ functional groups. Apart from functionalization through oxidized CNTs, the direct functionalization of CNTs is also possible. However, direct functionalization requires more reactive species to directly react with the CNTs, such as free radicals. Addition reactions to CNTs can cause a transformation from $\mathrm{sp}^{2}$ hybridization to $\mathrm{sp}^{3}$ hybridization at the point of addition. At the point where functionalization has taken place, the local bond geometry is changed from trigonal planar to tetrahedral geometry. Some addition reactions to the sidewalls of CNTs are shown in Fig. $16 .^{155}$

It is important to discuss how the covalent functionalization of carbon nanotubes comes at the price of the degradation of the carbon $\mathrm{sp}^{2}$ network. This substantially affects the electronic, thermal, and optoelectronic properties of the carbon nanotubes. $^{169}$ Efforts are being made to introduce a new method of covalent functionalization that can keep the $\pi$ network of CNTs intact. Antonio Setaro et al. introduced a new [2+1] cycloaddition reaction for the non-destructive, covalent, gram-scale functionalization of single-walled carbon nanotubes. The reaction rebuilds the extended $\pi$-network, and the carbon nanotubes retained their outstanding quantum optoelectronic properties (Fig. 17). ${ }^{173}$

5.1.2. Non-covalent functionalization. The non-covalent modification of CNTs is one of the simplest and most effective ways to functionalize the surface of CNTs to enhance their dispersibility

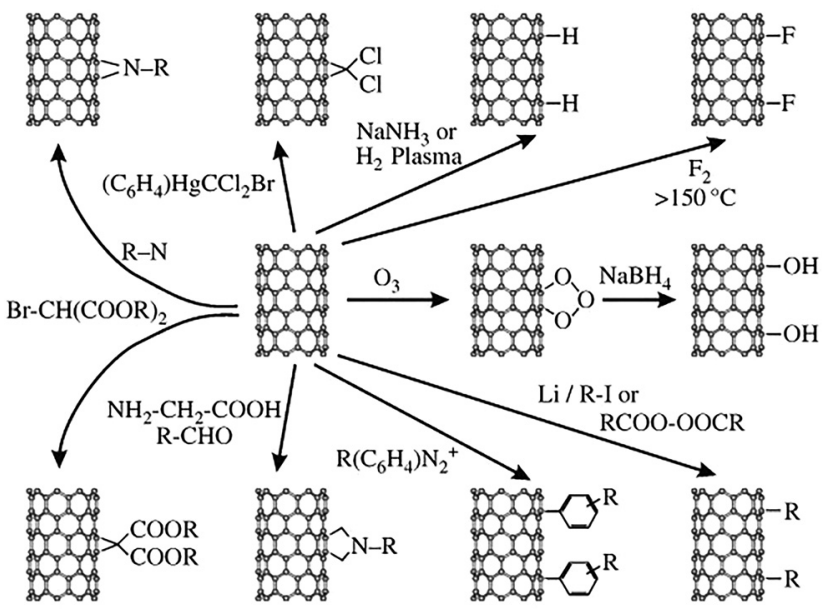

Fig. 16 An overview of possible addition reactions for the functionalization of nanotube sidewalls. Reprinted with permission from ref. 155. Copyright: (C2005, WILEY-VCH Verlag GmbH \& Co. KGaA, Weinheim.

and performance for relevant applications. The non-covalent functionalization of CNTs is achieved using $\pi-\pi$ interactions, $\mathrm{CH}-\pi$ interactions, van der Waals attraction, and electrostatic interactions. The non-covalent functionalization of CNTs is considered to be advantageous as it does not involve damaging the $\mathrm{sp}^{2}$ carbon network of CNTs and it helps to preserve the intrinsic characteristics of the CNTs. ${ }^{174}$ Different materials, such as polymers, aromatic compounds, and head-tail surfactants, are used for non-covalent functionalization. ${ }^{175}$

Polymers are frequently combined with CNTs to enhance their dispersion capabilities. Polymers interact with CNTs through $\mathrm{CH}-\pi$ and $\pi-\pi$ interactions. ${ }^{174}$ Hexanes and cycloalkanes are poor CNT solvents but the good solubility or dispersion of CNTs in these solvents is required for surface coating applications. Poly(dimethylsiloxane) (PDMS) macromer-grafted polymers have been prepared using PDMS macromers and pyrene-containing monomers that strongly adsorb on CNTs, thus improved the solubility of CNTs in chloroform and hexane. ${ }^{176}$ The use of head-tail surfactants is another attractive way to achieve a fine dispersion of CNTs in an aqueous medium. In head-tail surfactants, the tail is hydrophobic and interacts with the CNT sidewalls, and the hydrophilic head groups interact with the aqueous environment to provide a fine dispersion. ${ }^{177}$

For electrical applications, non-covalently functionalized CNTs are more preferred because the electrical properties of the CNTs are not compromised. CNTs have been non-covalently functionalized with a variety of biomolecules for the fabrication of electrochemical biosensors. ${ }^{175}$ Non-covalently functionalized SWCNTs are used for energy applications. Single-walled carbon nanotubes (SWCNTs) have been non-covalently functionalized with 3d transition metal(II) phthalocyanines, lowering the potential of the oxygen evolution reaction by approximately $120 \mathrm{mV}$ compared with unmodified SWCNTs. ${ }^{178}$ The toxicity of pristine CNTs toward living organisms can be lessened via using surfactantfunctionalized CNTs. ${ }^{170}$ However, in some cases, during polymer non-covalent functionalization, the polymer may wrap CNT 
a

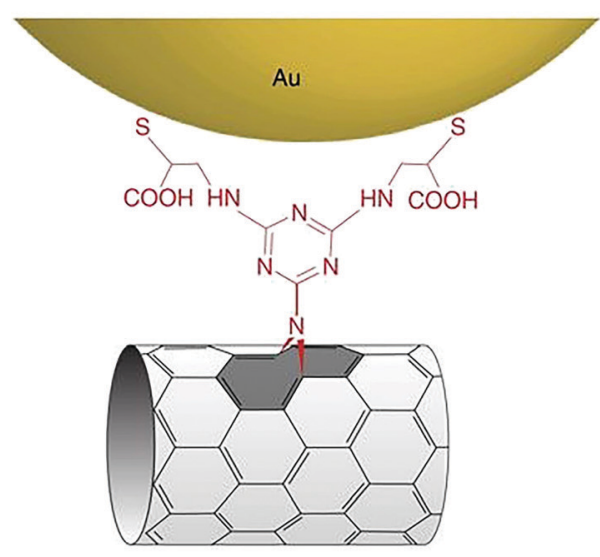

b

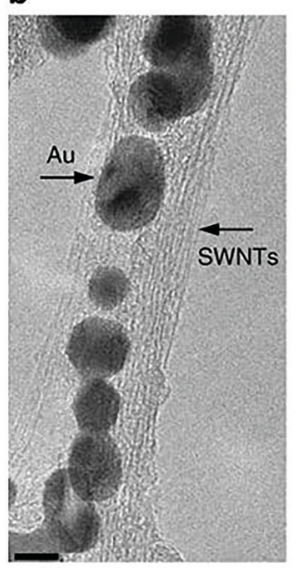

C

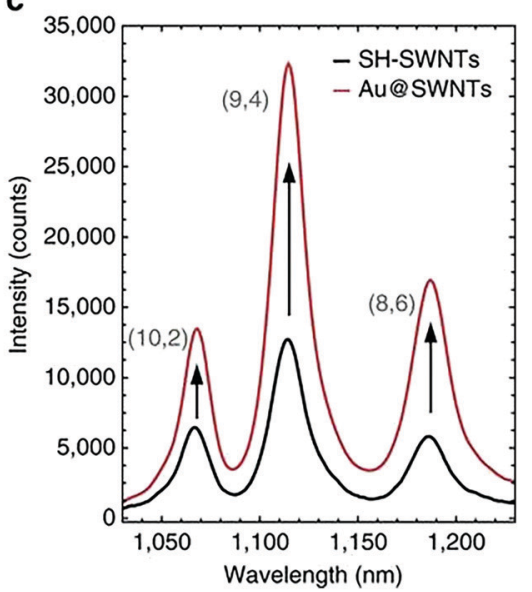

Fig. 17 (a) A molecular sketch of AuNPs covalently anchored to SH-SWNTs. (b) A TEM micrograph of the Au@SWNTs hybrid; scale bar: 5 nm; few-SWNT bundles can be observed, and AuNPs are assembled along the tubes. (c) The enhancement of the luminescence emission of SWNTs after the covalent attachment of AuNPs onto their surface: a comparison between the emission of Au@SWNTs hybrid (red curve) and that of SH-SWNTs (black curve). Reprinted with permission from ref. 173. Copyright: (C)2017, Springer Nature.

bundles and make it difficult to separate the CNTs from each other. Polymers can develop into insulating wrapping that affects the CNT conductivity.

5.1.3. Graphene. Graphene, a member of the carbon nanomaterial family, has emerged as a magic material that received incredible prominence within just a couple of years of its isolation from graphite in 2004. The striking features of graphene have promised to bring about revolutions in the fields of batteries, supercapacitors, solar cells, field-effect transistors, catalysis, sensors, and membrane technology. ${ }^{179-181}$ Graphene fever has continuously risen to try to meet the requirements of various devices and obtain desirable performance. Therefore, more pressure is being placed on this research to explore how to bring graphene to market. Graphene involves twodimensional $\mathrm{sp}^{2}$-hybridized carbon atom planar sheets that are tightly packed into honeycomb-like lattices. ${ }^{182}$ Graphene is one of the thinnest known materials and it possesses excellent mechanical strength. ${ }^{183}$ Graphene is transparent in nature, and that aspect of graphene is impressive when it comes to building transparent light panels and touch-screens. Graphene also possesses an exceptionally high theoretical surface area of about $2630 \mathrm{~m}^{2} \mathrm{~g}^{-1}$. The surface area of graphene is much higher compared to fullerenes and graphite. It is also higher compared to CNTs. S. Ghosh et al. ${ }^{184}$ reported that the thermal conductivity of graphene flakes is in the range of $\sim 3080-5150 \mathrm{~W} \mathrm{~m}^{-1} \mathrm{~K}^{-1}$. Alexander A. Balandin ${ }^{185}$ has obtained thermal conductivity of between $\sim(4.84 \pm 0.44) \times$ $10^{3}$ to $(5.30 \pm 0.48) \times 10^{3} \mathrm{~W} \mathrm{~m}^{-1} \mathrm{~K}^{-1}$ from single-layer graphene. This high value of thermal conductivity can outperform carbon nanotubes in the arena of heat conduction. K. I. Bolotin achieved ultrahigh electron mobility of $200000 \mathrm{~cm}^{2} \mathrm{~V}^{-1} \mathrm{~s}^{-1}$ at electron densities of $\sim 2 \times 10^{11} \mathrm{~cm}^{-2}$ via suspending single-layer graphene. ${ }^{186}$ The distinctive properties of graphene have attracted significant attention from researchers. They have started to evaluate it theoretically and experimentally in order to boost the performances of various devices via overcoming conventional challenges (Table 2).

In the literature, several graphene-related materials have been reported, such as graphene oxide and reduced graphene oxide. ${ }^{187}$ Among graphenoids, graphene oxide is a more reported and explored graphene-related material as a precursor for chemically modified graphene. The synthetic route to graphene oxide is straightforward, and it is synthesized from inexpensive graphite powder that is readily available. ${ }^{188}$ Graphene oxide has many oxygen-containing functional groups, such as epoxy, hydroxyl, carboxyl, and carbonyl groups. The basal plane of

Table 2 A comparison of the properties of graphene, graphene oxide, and reduced graphene oxide

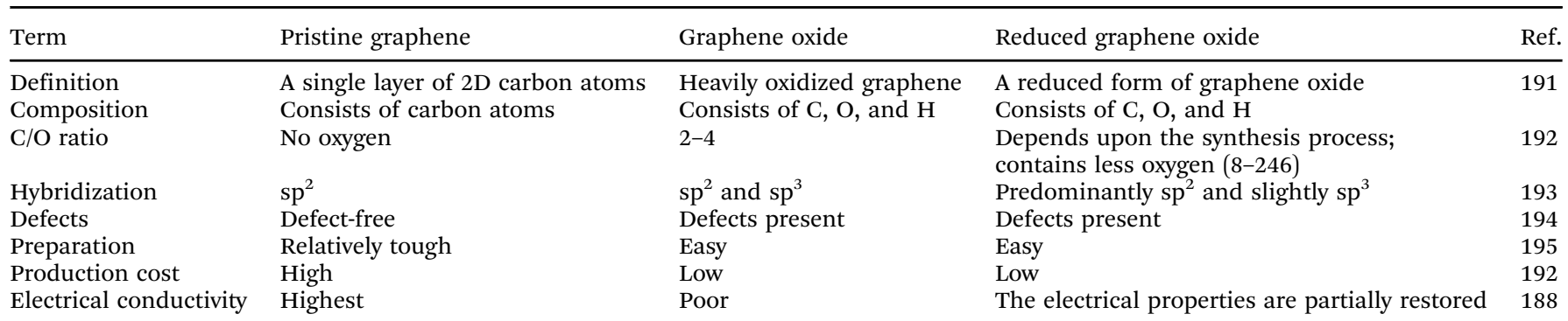


graphene oxide is generally decorated with epoxide and hydroxyl groups, whereas the edges presumably contain carboxyl- and carbonyl-based functional groups. ${ }^{189}$ The presence of active functional groups in graphene oxide allows its further functionalization with different polymers, small organic compounds, or other nanomaterials to realize several applications. ${ }^{190}$

Graphene oxide, due to its oxygen functionality, is insulating in nature and displays poor electrochemical performance. The presence of oxygen functionalities in graphene oxide breaks the conjugated structure and localizes the $\pi$-electron network, resulting in poor carrier mobility and carrier concentration. ${ }^{196}$ Its electrochemical performance is improved substantially after removing the oxygen-containing functional groups. ${ }^{197}$ These functional groups can be removed or reduced via thermal, electrochemical, and chemical means. The product obtained after removing or reducing oxygen moieties is called reduced graphene oxide. The properties of reduced graphene oxide depend upon the effective removal of oxygen moieties from graphene oxide. The process used to remove oxygen-containing functionalities from graphene oxide will determine the extent to which reduced the properties of graphene oxide resemble pristine graphene. ${ }^{198}$
Reduced graphene oxide is extensively used to improve the performances of various electrochemical devices. ${ }^{199}$ It is essential to mention that even after reducing graphene oxide, some residual $\mathrm{sp}^{3}$ carbon bonded to oxygen still exists, which somehow disturbs the movement of charge through the delocalized electronic cloud of the $\mathrm{sp}^{2}$ carbon network. ${ }^{200}$ Apart from this, the electrochemical activity of reduced graphene oxide is substantially high enough to manufacture electrochemical devices with improved performances. Recently, the demand for superperformance electrochemical devices has increased to overcome modern challenges relating to electronics and energy-storage devices. ${ }^{201}$ Graphene-based materials are considered to be excellent electrode materials, and they can be proved to be revolutionary for use in energy-storage devices such as supercapacitors (SC) and batteries. Graphene-based electrodes improve the performances of existing batteries (lithium-ion batteries) and they are considered useful for developing next-generation batteries such as sodiumion batteries, lithium- $\mathrm{O}_{2}$ batteries, and lithium-sulfur batteries (Fig. 18). Being flat in nature, each carbon atom of graphene is available, and ions can easily access the surface due to low diffusion resistance, which provides high electrochemical activity. ${ }^{202}$

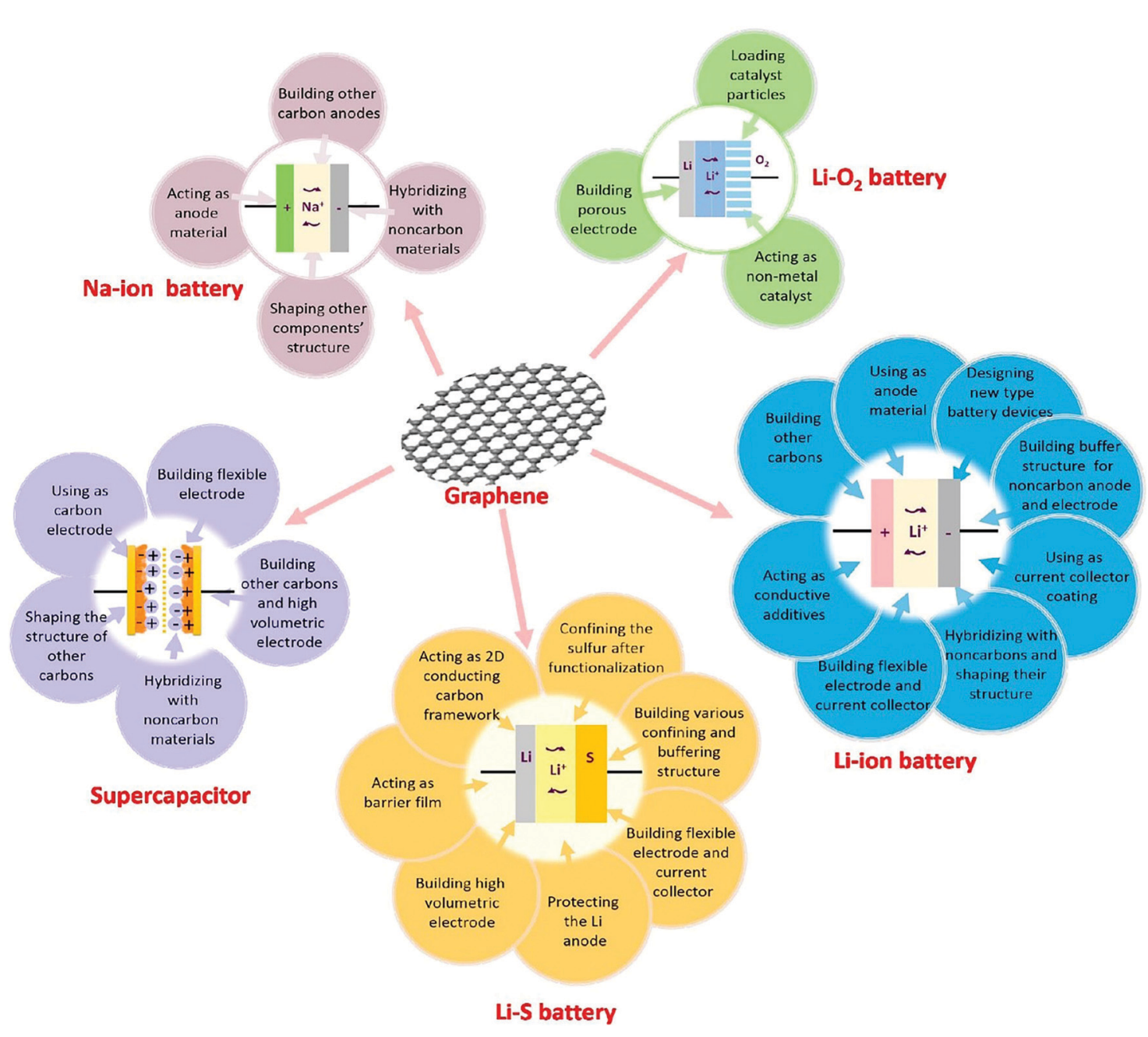

Fig. 18 The applications of graphene in different electrochemical energy storage devices (EESDs). Reprinted with permission from ref. 202. Copyright: (C)2015, Elsevier B.V. All rights reserved. 
Graphene and its derivatives are extensively used for the development of electrochemical sensors. ${ }^{203}$ The surfaces of bare electrodes are usually not able to sense analytes at trace levels and they cannot differentiate between analytes that have close electrooxidation properties due to their poor surface kinetics. The addition of graphene layers to the surfaces of electrodes can substantially improve the electrocatalytic activity and surface sensitivity towards analytes. ${ }^{204}$ Graphene has definite advantages over other materials that are used as electrode materials for sensor applications. Graphene has a substantially high surface-to-volume ratio and atomic thickness, making it extremely sensitive to any changes in its local environment. This is an essential factor in developing advanced sensing tools, as all the carbon atoms are available to interact with target species.

Consequently, graphene exhibits higher sensitivity than its counterparts such as CNTs and silicon nanowires. ${ }^{205}$ Graphene has two main advantages over CNTs for the development of electrochemical sensors. First, graphene is mostly produced from graphite, which is a cost-effective route, and second, graphene does not contain metallic impurities like CNTs can. Graphene offers many other advantages when developing sensors and biosensors, such as biocompatibility and $\pi-\pi$ stacking interactions with biomolecules. ${ }^{206}$ Graphene-based materials are ideal for the construction of nanostructured sensors and biosensors.

The mechanical properties of graphene are used to fabricate highly desired stretchable and flexible sensors. ${ }^{207}$ Graphene can be utilized to develop transparent electrodes with excellent optical transmittance. It displays good piezoresistive sensitivity. Researchers are making efforts to replace conventional brittle indium tin oxide (ITO) electrodes with flexible graphene electrodes in optoelectronic devices such as liquid-crystal displays and organic light-emitting diodes. ${ }^{208}$ For human-machine interfaces, transparent and flexible tactile sensors with high sensitivity have become essential. Graphene film (GF) and PET have been applied to develop transparent tactile sensors that exhibit outstanding cycling stability, fast response times, and excellent sensitivity (Fig. 19). ${ }^{209}$ Similarly, graphene is applied for the fabrication of pressure sensors. ${ }^{210}$ Overall, graphene is an excellent material for developing transparent and flexible devices. ${ }^{211,212}$

The use of graphene-based materials is an effective way to deal with a broad spectrum of pollutants. ${ }^{213}$ There are many ways to deal with environmental pollution; among these, adsorption is an effective and cost-effective method. ${ }^{21,215}$ Graphene-based adsorbents are found to be useful in the removal of organic, ${ }^{216}$ inorganic, and gaseous contaminants. Graphene-based materials have some obvious advantages over CNT-based adsorbents. For example, graphene sheets offer two basal planes for contaminant adsorption, enhancing their effectiveness as an adsorbent. ${ }^{192} \mathrm{GO}$ contains several oxygen functional groups that impart hydrophilic features. Due to appropriate hydrophilicity, GO-based adsorbents can efficiently operate in water to remove contaminants. Moreover, graphene-oxide-based materials can be functionalized further through reactive moieties with various organic molecules to enhance their adsorption capacities. ${ }^{217}$

In short, extensive research must continue in order to develop graphene-based materials with high performance and
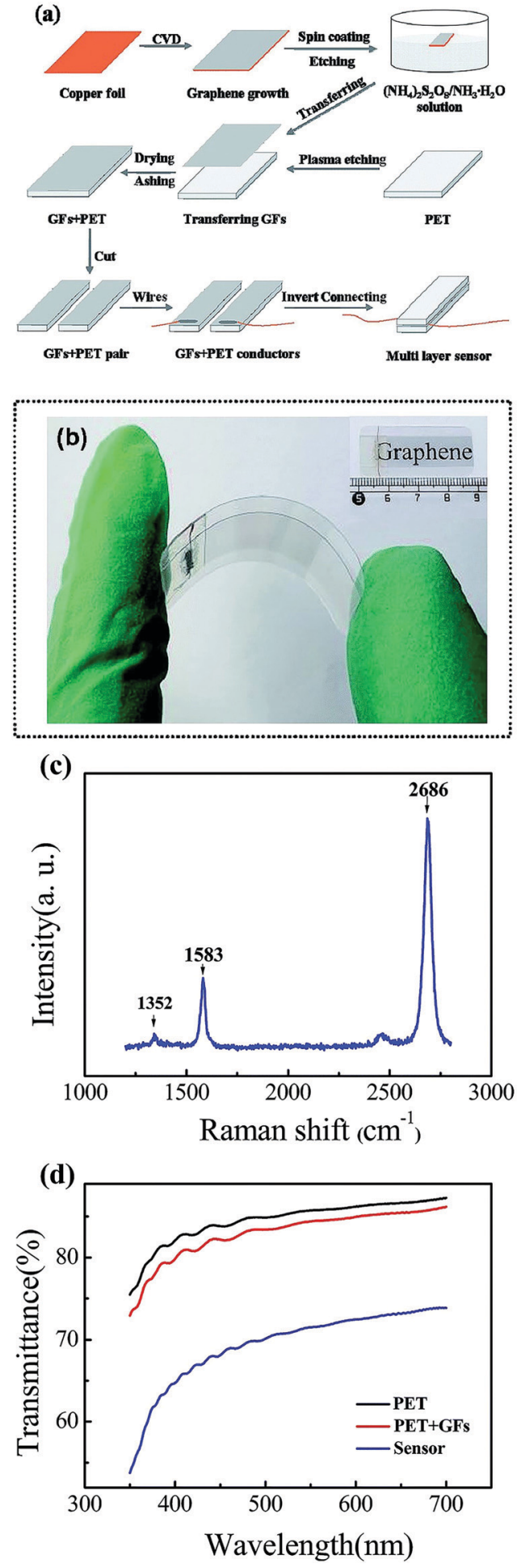

Fig. 19 (a) A schematic diagram of the fabrication procedure of a tactile sensor based on GF and a PET plate. (b) An optical photograph of a bent assembled sensor; the geometrical dimensions of the sensor are shown in the inset. (c) The Raman spectrum of the GF, with typical D $\left(\approx 1352 \mathrm{~cm}^{-1}\right)$, $\mathrm{G}\left(\approx 1583 \mathrm{~cm}^{-1}\right)$, and $2 \mathrm{D}\left(\approx 2686 \mathrm{~cm}^{-1}\right)$ peaks. (d) Transmittance spectra of pure PET, the GF-PET composite structure, and a multilayer stacked nanofilm sensor in the visible wavelength range from 350 to $700 \mathrm{~nm} .{ }^{209}$ Published by The Royal Society of Chemistry 
bring them to the market. Massive focus on graphene research is also justified due to the extraordinary features described in extensive theoretical and experimental research works.

5.1.4. Nanodiamonds. Like other carbon-based materials, nanodiamonds possess numerous exciting properties that make them attractive nanomaterials for a wide range of applications. Nanoscale diamonds first appeared on the surface in the 1960s as a result of explosives research in the USSR. ${ }^{218}$ However, these nanomaterials remained unfamiliar to the world until the end of the 1980s, and critical breakthroughs were not observed until the beginning of the late 1990 s. $^{219}$ The term nanodiamond is used for monocrystalline diamonds whose particle size is less than $100 \mathrm{~nm}^{220}$ Nanodiamonds consist of $\mathrm{sp}^{3}$-hybridized carbon nanoparticles. Nanodiamonds are unique carbon-based nanomaterials and they exhibit exceptional optical properties, mechanical properties, high specific surface areas, and rich surface structures. Nanodiamonds can be synthesized using several methods, such as the ion irradiation of graphite, ${ }^{221}$ high-energy ball milling, ${ }^{222}$ carbide chlorination, ${ }^{223}$ chemical vapor deposition, ${ }^{224}$ and laser ablation. ${ }^{225}$

Nanodiamonds possess a core-shell-like structure and display rich surface chemistry, and numerous functional groups are present on their surface. Several functional groups, such as amide, aldehyde, ketone, carboxylic acid, alkene, hydroperoxide, nitroso, carbonate ester, and alcohol groups, are present on nanodiamond surfaces, assisting in their further functionalization for desired applications (Fig. 20). ${ }^{226}$

Furthermore, nanodiamond surfaces can be homogenized with a single type of functional group according to the application requirements. ${ }^{227}$ The use of nanodiamond particles as a reinforcing material in polymer composites has attracted great attention for improving the performance of polymer composite materials. The superior mechanical properties and rich surface chemistry of nanodiamonds have made them a superior material for tuning and reinforcing polymer composites. Nanodiamonds might operate via changing the interphase properties and forming a robust covalent interface with the matrix. ${ }^{228}$ Nanodiamond (ND)-reinforced polymer composites have shown superior thermal stabilities, mechanical properties, and thermal conductivities. Nanodiamonds have shown great potential for energy storage applications. ${ }^{229}$ Nanodiamonds and their composites are also used in sensor fabrication, environmental remediation, and wastewater treatment. ${ }^{230,231}$ Their stable fluorescence and long fluorescence lifetimes have made nanodiamonds useful for imaging and cancer treatment. For biomedical applications, the rational engineering of nanodiamond particle surfaces has played a crucial role in the carrying of bioactive substances, target ligands, and nucleic acids, resisting their aggregation. ${ }^{232,233}$ Nanodiamonds have a great future in nanotechnology due to their amazing surface chemistry and unique characteristics.

5.1.5. Carbon-based quantum dots. Carbon quantum dots are a new form of nanocarbon and they were accidentally formed during the electrophoretic purification of single-walled carbon nanotubes in 2004. ${ }^{234}$ Carbon quantum dots are zerodimensional discrete and quasi-spherical nanoscale particles of carbon that are less than $10 \mathrm{~nm}$ in size. ${ }^{235}$ Graphene nanosheets that are less than $100 \mathrm{~nm}$ in lateral size are generally described as graphene quantum dots. Ideally, they involve a single atomic layer of nano-sized graphite. ${ }^{235,236}$ Carbon quantum dots possess several unique features that make them extraordinary materials for several applications, such as: $:^{237-240}$ (i) tunable photoluminescence properties and good multi-photon excitation; (ii) biocompatibility and low toxicity; (iii) some distinctive features relating to quantum confinement effects; (iv) solubility in water due to

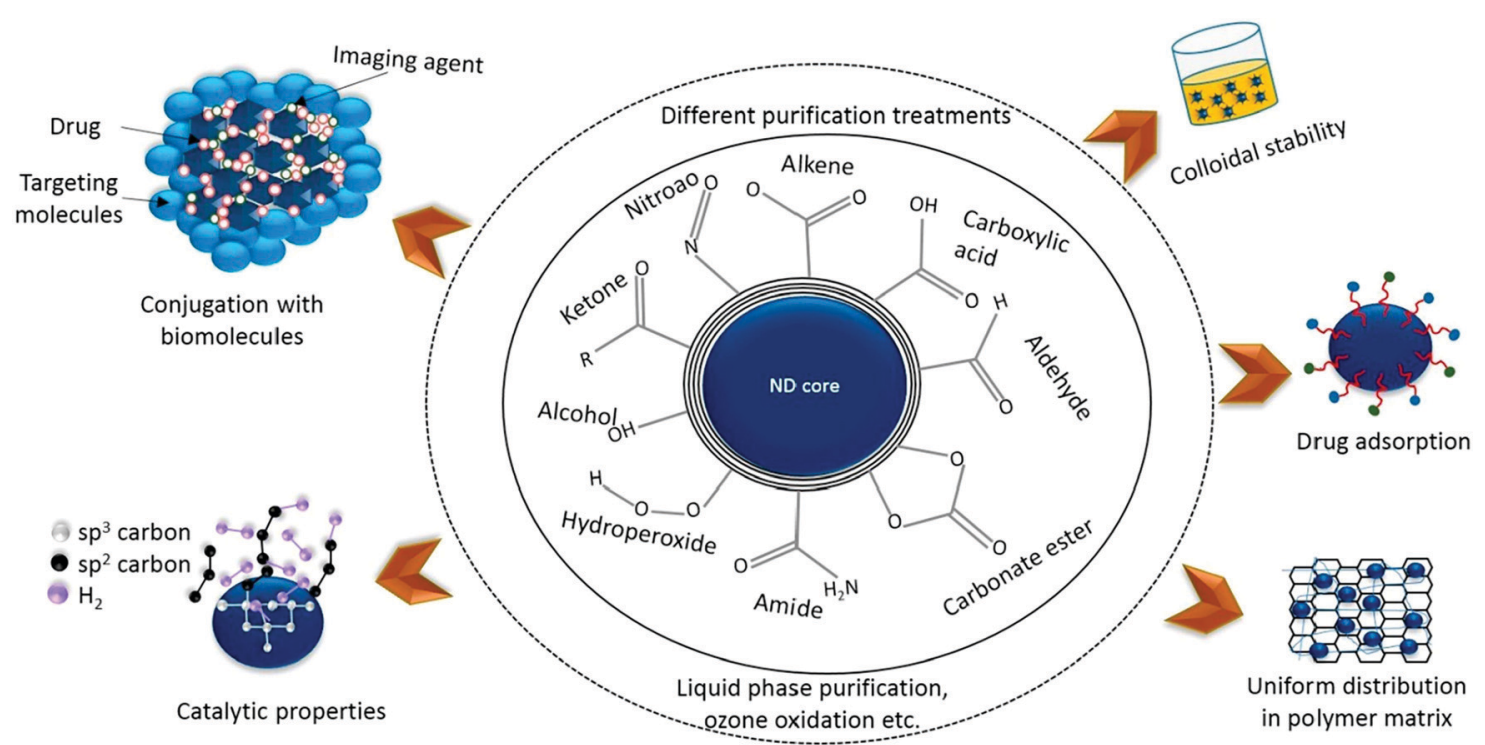

Fig. 20 The critical surface chemistry of detonation-based NDs. The ND surface is usually covered with several functional groups. Different surface treatments (such as liquid phase purification and ozone oxidation) are needed to replace these functional groups with oxygen-containing species like carboxylic acids and anhydrides. Surface-treated NDs exhibit several attractive properties, such as colloidal stability, drug adsorption, uniform distribution in a polymer matrix, conjugation with biomolecules, and catalytic properties. Reprinted with permission from ref. 226. Copyright: (C)2018, Elsevier Ltd. All rights reserved. 
rich oxygen-containing groups; and (v) cost effectiveness and environmental friendliness.

Carbon quantum dots can be synthesized through several chemical routes. $^{241-245}$ Some methodologies for synthesizing carbon dots are described in Fig. 21. ${ }^{246-248}$ Carbon itself is a black material and displays low solubility in water. In contrast, carbon quantum dots are attractive due to their excellent solubility in water. They contain a plethora of oxygen-containing functional groups on their surface, such as carboxylic acids. These functional moieties allow for further functionalization with biological, inorganic, polymeric, and organic species.

Carbon quantum dots are also called carbon nano-lights due to their strong luminescence. ${ }^{248}$ In particular, carbon quantum dots offer enhanced chemiluminescence, ${ }^{249,250}$ fluorescent emission, ${ }^{251}$ two-photon luminescence under near-infrared pulsed-laser excitation, ${ }^{252}$ and tunable excitation-dependent fluorescence. ${ }^{253}$ The luminescence characteristics of carbon quantum dots have been used to develop highly sensitive and selective sensors. In most cases, a simple principle is involved in sensing with luminescent carbon quantum dots: their photoluminescence intensity changes upon the addition of an analyte. ${ }^{254}$ Based on this principle, several efficient sensors have been developed using carbon quantum dots. ${ }^{255-257}$ They can be used as sensitive and selective tools for sensing explosives such as TNT. Recognition molecules on the surfaces of carbon quantum dots can help to sense targeted analytes. Amino-group-functionalized carbon quantum dot fluorescence is quenched in the presence of TNT through a photo-induced electron-transfer effect between TNT and primary amino groups. This quenching phenomenon can help to sense the target analyte (Fig. 22). ${ }^{258}$ Chiral carbon quantum dots (cCQDs) can exhibit an enantioselective response. The PL responses of cCQDs were evaluated toward 17 amino acids and it was found that the PL intensity of the cCQDs was only substantially enhanced in the presence of L-Lys (Fig. 22). ${ }^{254}$

Carbon quantum dots have received significant interest in the fields of biological imaging and nanomedicine (Fig. 23). ${ }^{239}$ Direct images of RNA and DNA are essential for understanding cell anatomy. Due to the limitations of current imaging probes, tracking the dynamics of these biological macromolecules is not an easy job. Recently, membrane-penetrating carbon quantum dots have been developed for the imaging of nucleic acids in live organisms. $^{259}$ It is important to note that most of the carbon quantum dots utilized to attain cell imaging under UV excitation emit blue radiation. Some biological tissue also emits blue light, specifically that involving carbohydrates, and this interferes with cell imaging carried out with blue-emitting CQDs. This seriously hinders their potential in the field of biomedical imaging. Due to this reason, researchers are focusing on tuning CQDs in a way that their emission peak is red-shifted to avoid interference. ${ }^{260}$ Carbon quantum dots with yellow and green fluorescence have been reported for bioimaging purposes. ${ }^{261,262}$ The suitable doping of carbon quantum dots can red-shift the emission to enhance the bioimaging effectiveness. ${ }^{263}$ Doped carbon quantum dots are capable of biological imaging and display advanced capabilities for scavenging reactive oxygen species. ${ }^{264}$

Carbon quantum dots demonstrate photo-induced electron transfer properties ${ }^{265}$ that make them valuable for photocatalytic, light-energy conversion, and other related applications. ${ }^{266}$ Carbon quantum dots enhance the activities of other photocatalysts to which they are attached. Carbon quantum dots, along with photocatalysts, provide better charge separation and suppress

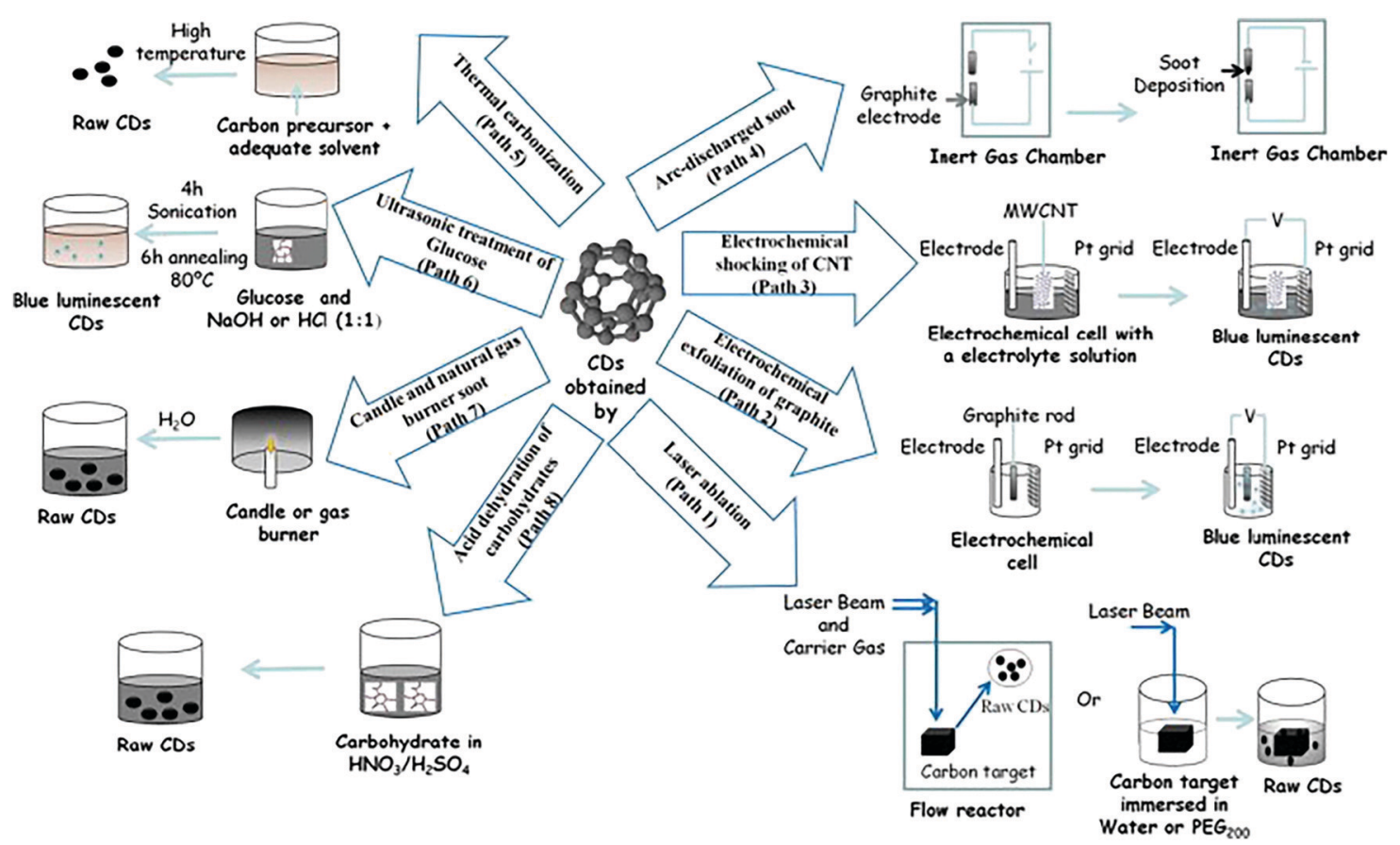

Fig. 21 Methodologies for synthesizing carbon dots (CDs). Reprinted with permission from ref. 246. Copyright: (C)2011, Elsevier Ltd. All rights reserved. 


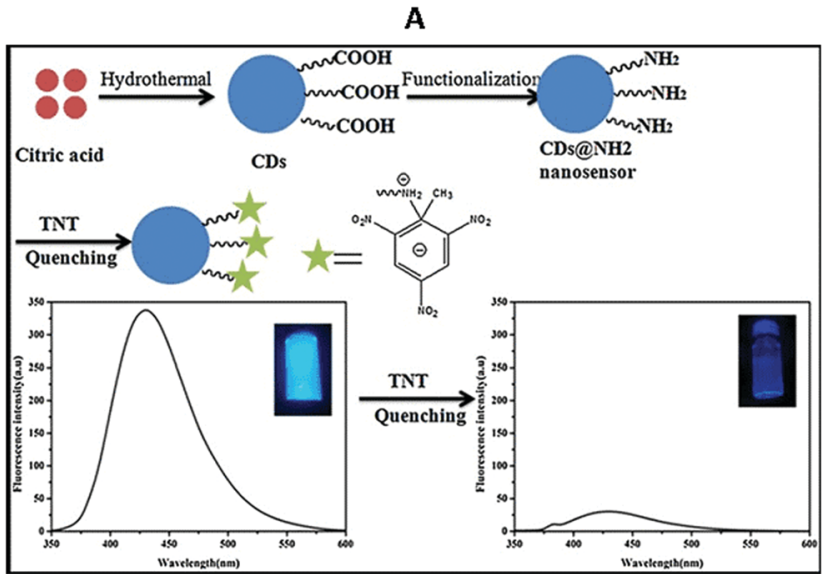

B

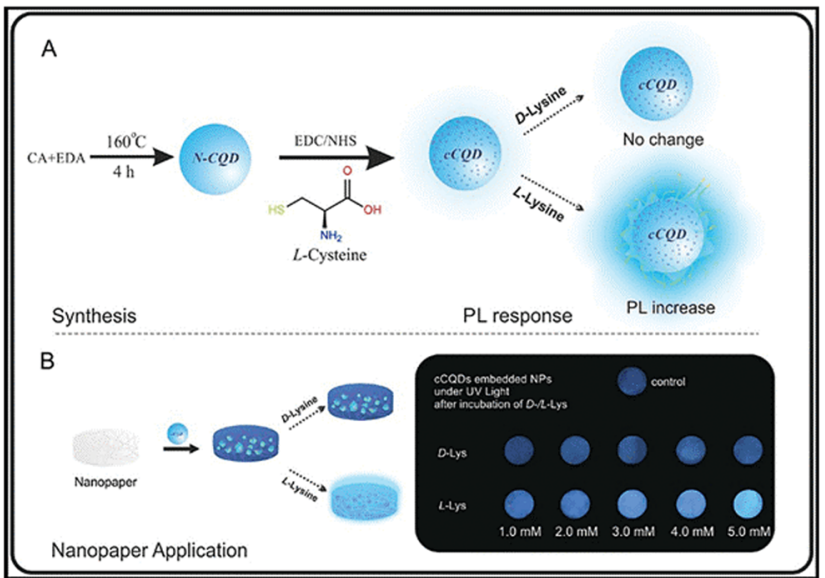

Fig. 22 (A) A schematic illustration of the fabrication of a $\mathrm{CDs}_{\mathrm{aNH}}$ nanosensor and its sensing mechanism toward TNT. ${ }^{258}$ (B) A schematic diagram of the synthesis and application procedures of CCQDs. Reprinted with permission from ref. 254. Copyright: (C)2018, Elsevier B.V. All rights reserved.

the regeneration of photogenerated electron-hole pairs. Moreover, the proper implantation of carbon quantum dots into

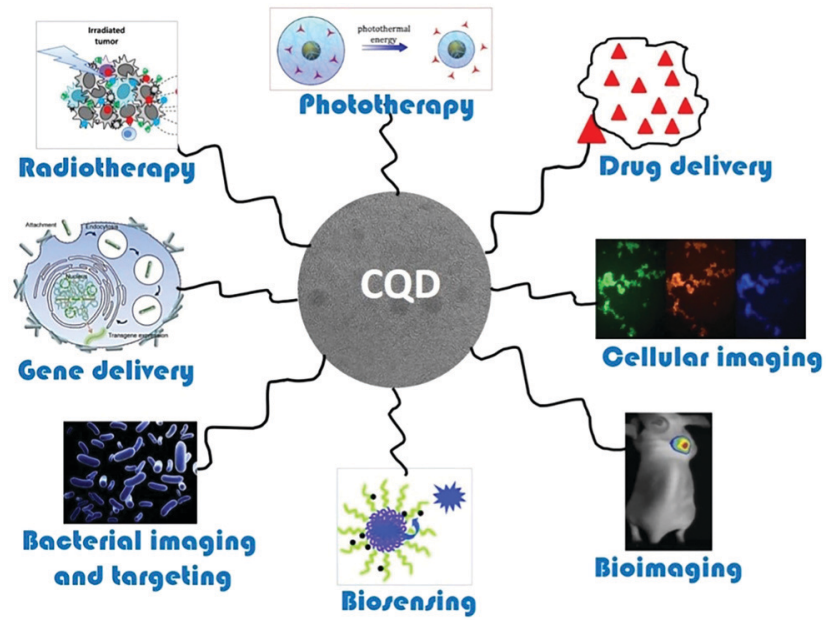

Fig. 23 The applications of CQDs in nanomedicine. Reprinted with permission from ref. 239. Copyright: (C2019, Elsevier B.V. All rights reserved. photocatalysts can broaden the photo-absorption region. Implanted carbon quantum dots form micro-regional heterostructures that facilitate photo-electron transport. ${ }^{267}$ The implantation of carbon quantum dots into $\mathrm{g}-\mathrm{C}_{3} \mathrm{~N}_{4}$ can substantially enhance charge transfer and separation efficiencies, prevent photoexcited carrier recombination, narrow the bandgap, and red shift the absorption edge. ${ }^{268}$ The intrinsic catalytic activity of polymeric carbon nitride is improved as a result of the nanoframe heterojunctions formed with the help of CQDs. ${ }^{269}$

Carbon quantum dots offer many advantages over conventional semiconductor-based QDs and, thus, they have attracted considerable researcher attention. ${ }^{244}$ Due to their remarkable features, they have shown importance in recent years in the fields of light-emitting diodes, nanomedicine, solar cells, sensors, catalysis, and bioimaging. ${ }^{236}$

5.1.6. Carbon nanohorns/nanocones. Carbon nanohorns are also known as carbon nanocones. They appear as conical carbon nanostructures that consist of $\mathrm{sp}^{2}$ carbon sheets. Singlewalled carbon nanohorns consist of a tubular structure with a graphene sheet that has a conical end. Due to their closed cage structure, they are considered a subset of fullerenes, and their elongated shape makes them a structural analogue to short single-walled carbon nanotubes. Carbon nanohorns have lengths of $40-50 \mathrm{~nm}$ with diameters in the range of $2-5 \mathrm{~nm} \cdot{ }^{270}$ The assembly of thousands of SWNHs results in nanohorn aggregate formation with a diameter of $\sim 80-100 \mathrm{~nm} .{ }^{271}$ The aggregation of carbon nanohorns can be observed in Fig. $24 .^{272}$ Three different types of carbon nanohorns have been observed: ${ }^{273}$ dahlia-like CNHs; bud-like CNHs; and seed-like CNHs.

The production of carbon nanohorns has some obvious advantages over carbon nanotubes, such as the ability for toxic-metal-catalyst-free synthesis and large-scale production at room temperature. Carbon nanotube synthesis involves metal particles, and harsh conditions, such as the use of strong acids, are required to remove metallic catalysts. This process introduces many defects into CNT structures and may cause a loss of carbon material. ${ }^{270}$ Carbon nanohorns possess a wide diameter compared to CNTs. CNHs possess good absorption capabilities and their interiors are also available after partial oxidation, which provides direct access to their internal parts. Heat treatment under acidic or oxidative conditions facilitates the facile introduction of holes into carbon nanohorns. Holes in graphene sheets of single-walled carbon nanohorns can be produced with $\mathrm{O}_{2}$ gas at high temperatures. A large quantity of material can be stored inside $\mathrm{CNH}$ tubes. ${ }^{274}$ The surface area of CNHs is substantially enhanced upon opening the horns to make their interiors accessible. ${ }^{275}$ Carbon nanohorns have great potential for energy storage ${ }^{275}$ electrochemiluminescence, ${ }^{276}$ adsorption, ${ }^{277}$ catalyst support, ${ }^{278}$ electrochemical sensing, ${ }^{279}$ and drug delivery system ${ }^{273}$ uses. CNHs as catalyst supports can provide a homogeneous dispersion of Pt nanoparticles (Fig. 25). The current density of Pt supported on single-walled carbon nanohorns is double compared to a fuel cell made from Pt supported on carbon black. $^{280}$ Thus, carbon nanohorns provide a better uniform dispersion that facilitates a high surface area and better catalyst performance. 

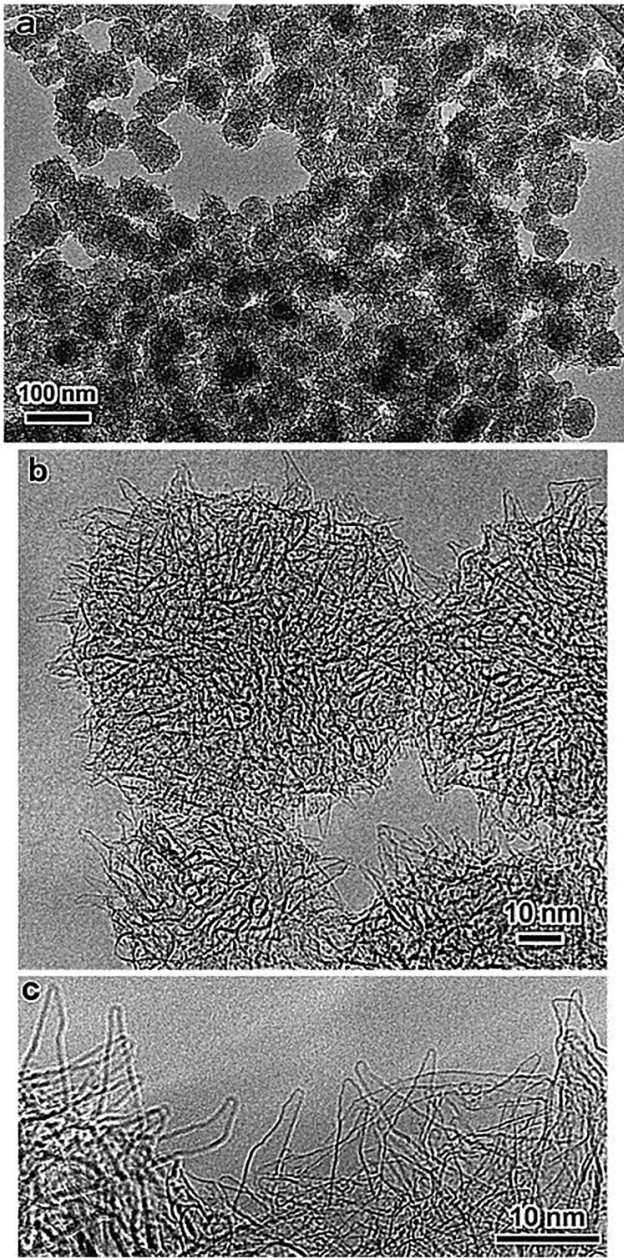

Fig. 24 (a) A TEM micrograph showing a graphitic carbon product that was generated abundantly upon $\mathrm{CO}_{2}$ laser ablation at room temperature. The product consisted of near-uniform-sized spherical particles with a diameter of $80 \mathrm{~nm}$. (b) A magnified TEM micrograph of the graphitic carbon particles showing aggregations of tubule-like structures sticking out of the particle surface. (c) A highly magnified TEM micrograph of the edge regions of graphitic particles showing conical horn-like protrusions that are up to $20 \mathrm{~nm}$-long on the particle surface with some modified shapes. Each of these carbon nanohorns was made of a single graphene sheet with closed caps, and the diameters were similar to those of fullerene molecules. Reprinted with permission from ref. 272. Copyright: (C)1999, Elsevier Science B.V. All rights reserved.

\subsection{Nanoporous materials}

Explorations and research relating to the development of porous materials have fascinated materials chemists for decades. ${ }^{281}$ One example of a porous materials is activated charcoal, which has been used for many years. ${ }^{282}$ The technological and scientific importance of porous solids arises from their ability to interact with ions, atoms, and molecules at their outer surface, while their interior is also accessible. Porous materials are generally defined as nanoporous materials when they have a pore diameter of less than $100 \mathrm{~nm} .^{283,284}$ According to the pore size, porous materials can be divided into three major categories: ${ }^{285,286}$

5.2.1. Macroporous materials. Porous materials are recognized as macroporous when they have pore sizes greater than $50 \mathrm{~nm}$.
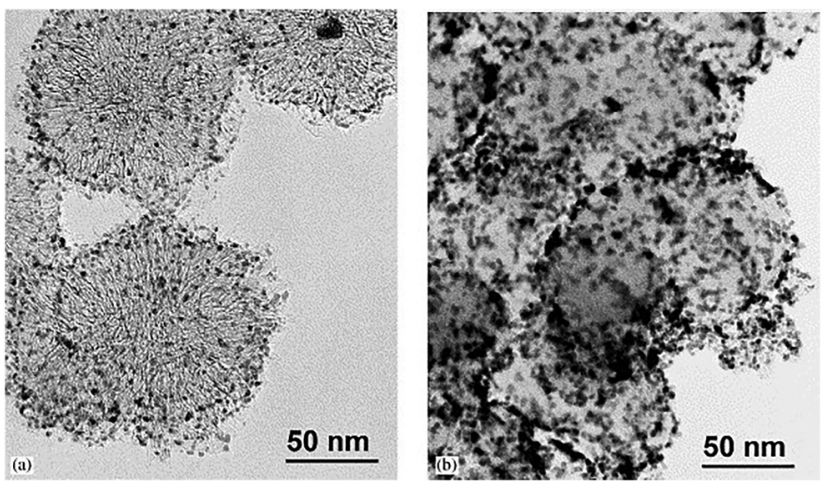

Fig. 25 TEM micrographs of a Pt catalyst supported on SWNHs (a) and on carbon black (b). Reprinted with permission from ref. 280. Copyright: (c)2002, Elsevier Science B.V. All rights reserved.

5.2.2. Mesoporous materials. Porous materials are called mesoporous when they have pores in the range of 2 to $50 \mathrm{~nm}$.

5.2.3. Microporous materials. Microporous materials have pore sizes in the range of $2 \mathrm{~nm}$.

In nanoporous materials, the size distributions, volumes, and shapes of the pores directly affect the performances of porous materials for particular applications. It has become a hot area of research to develop materials with precisely controlled pores and arrangements. Recent research has focused more on the precise control of the shapes, sizes, and volumes of pores to produce nanoporous materials with high performance. Several state-of-theart reviews are present in the literature that focus explicitly on the synthesis, properties, advances, and applications of nanoporous materials. ${ }^{85,287-289}$ Based on the materials used, nanoporous materials can be divided into three main groups: inorganic nanoporous materials; carbonaceous nanoporous materials; and organic polymeric nanoporous materials.

Inorganic nanoporous materials include porous silicas, clays, porous metal oxides, and zeolites. The generation of pores in the material can introduce striking features into the material that are absent in non-porous materials. Nanoporous materials offer rich surface compositions with versatile characteristics. Nanoporous materials exhibit high surface-to-volume ratios. Their outstanding features and nanoporous framework structures have made these materials valuable in the fields of environmental remediation, adsorption, catalysis, sensing, energy conversion, purification, and medicine. ${ }^{284,290}$

Porous silica is a crucial member of the inorganic nanoporous family. Over the decades, it has generated significant research interest for use in fuel cells, chemical engineering, ceramics, and biomedicine. It is essential to note that specific morphologies and pore size diameters are required for each application, and these can be achieved via tuning during the synthesis process. Nanoporous silica offers two functional surfaces: one is the cylindrical pore surfaces, and the second is the exterior surfaces of the nanoporous silica particles. The surfaces of nanoporous silica can be easily functionalized for the desired applications. The nanoporous silica surface is heavily covered with many silanol groups that act as reactive sites for functionalization (Fig. 26). ${ }^{291,292}$ For biomedical applications, mesoporous silica has emerged as a 


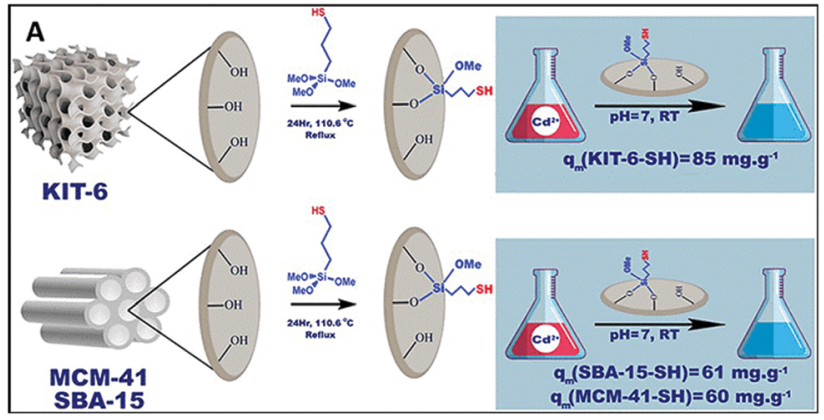

B

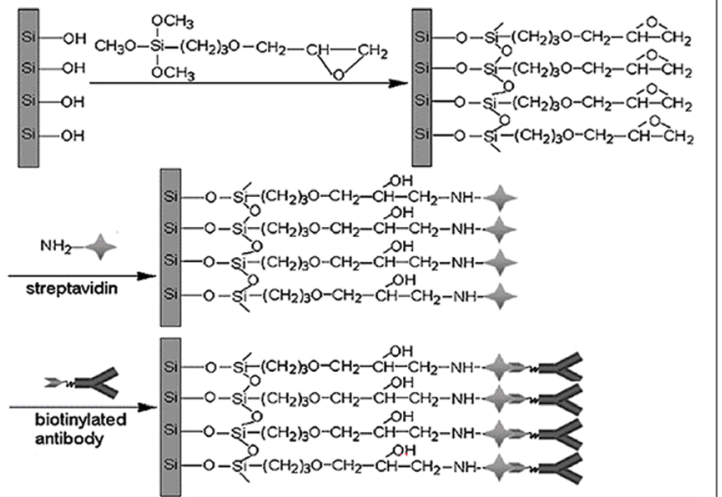

Fig. 26 (A) The functionalization of mesoporous silica and the adsorption process of cadmium ions. Reprinted with permission from ref. 292. Copyright: (C)2018, Elsevier B.V. All rights reserved. (B) A schematic illustration of the biofunctionalization of 3D nanoporous $\mathrm{SiO}_{2}$ film with streptavidin and antibodies. Reprinted with permission from ref. 291. Copyright: (C)2008, WILEY-VCH Verlag GmbH \& Co. KGaA, Weinheim.

new generation of inorganic platform materials compared to other integrated nanostructured materials. Several factors make it a unique material for biomedical applications: ${ }^{293,294}$ (a) its ordered porous structure; (b) its tunable particle size; (c) its large pore volume and surface area; (d) its biocompatibility; (e) its biodegradation, biodistribution, and excretion properties; and (f) its two functional surfaces. For instance, ordered MCM-48 nanoporous silica was used for the delivery of the poorly soluble drug indomethacin. It has been found that surface modification can control drug release. ${ }^{295}$ Mesoporous silica-based materials have emerged as excellent materials for use in sustained drug delivery systems (SDDSs), immediate drug delivery systems (IDDSs), targeted drug delivery systems (TDDSs), and stimuli-responsive controlled drug delivery systems (CDDSs). The drug release rate from mesoporous silica can also be controlled via introducing appropriate polymers or functional groups, such as $\mathrm{CN}, \mathrm{SH}, \mathrm{NH}_{2}$, and $\mathrm{Cl}$. Researchers are currently focusing on developing MSN-based (MSN = mesoporous silica nanoparticle) multifunctional drug delivery systems that can release antitumor drugs on demand in a targeted fashion via minimizing the premature release of the drug (Fig. 27). ${ }^{296}$

Hierarchically nanoporous zeolites are a vital member of the nanoporous material family. They are crystalline aluminosilicate minerals whose structures comprise uniform, regular arrays of nanopores with molecular dimensions. The microporous structures of zeolites contain pores that are usually below $1 \mathrm{~nm}$ in diameter.
In zeolites, the micropores are uniform in shape and size, and these pores can effectively discriminate between molecules based on shape and size. ${ }^{297}$ Currently, based on crystallography, more than 200 zeolites have been classified. ${ }^{298}$ Zeolites have been proved to be useful materials in the field of host-guest chemistry. In solid catalysis, about $40 \%$ of the entire solid catalyst field is taken up by zeolites in chemical industry. The excellent catalysis success of zeolites is based on their framework stability, shape-selective porosity, solid acidity, and ion-exchange capacity. Oxygen tetrahedrally coordinates with the $\mathrm{Al}$ atoms in most zeolite crystalline silicate frameworks, resulting in charge mismatch between the oxide framework and Al. Extra-framework $\mathrm{Na}^{+}$ions compensate for this charge mismatch. The $\mathrm{Na}^{+}$ions are exchangeable for other cations such as $\mathrm{H}^{+}$and $\mathrm{K}^{+} .{ }^{298}$ The zeolite crystalline networks are remarkable in that they provide high mechanical and hydrothermal stabilities. The most crucial task facing the zeolite community is to find new structures with desired functions and apply them more effectively for different applications.

Apart from these inorganic porous materials, several other metal- and metal-oxide-based nanoporous materials have been introduced that are more prominent for use in electrode material, catalyst, photodegradation, energy storage, and energy conversion applications. ${ }^{299-302}$ Nanoporous metal-based materials are famous due to the nanosized crystalline walls, interconnected porous networks, and numerous surface metal sites that provide them with unique physical/chemical properties compared with their bulk counterparts and other nanostructured materials. ${ }^{303}$ For example, nanoporous $\mathrm{WO}_{3}$ films were developed via tuning the anodization conditions for photoelectrochemical water oxidation. It has been observed that the morphology of the film strongly affected the photoelectrochemical performance. ${ }^{304}$ Nanoporous alumina is also a unique material in the inorganic nanoporous family due to several aspects. Nanoporous alumina can be prepared in a controlled fashion with any size and shape in polyprotic aqueous media via the anodic oxidation of the aluminum surface. The parallel arrangement of pores on alumina can easily be controlled from $5 \mathrm{~nm}$ to $300 \mathrm{~nm}$, and alumina is stable in the range of $1000{ }^{\circ} \mathrm{C}$. The anodizing time plays a significant role in controlling the pore length. Nanoporous alumina membranes offer various unique properties, such as pores of variable widths/ lengths, temperature stability, and optical transparency. Nanoporous alumina pores can be filled with magnetically and optically active elements to produce the desired applications at the nanoscale level. Photoluminescent alumina membranes can be produced via introducing cadmium sulfide, gallium nitride, and siloxenes inside nanoporous alumina using appropriate precursors. ${ }^{305}$ Porous alumina also acts as an efficient support and template for the designing of other nanomaterials. Palladium nanowires, ${ }^{306}$ high aspect ratio cobalt nanowires, ${ }^{307}$ and highly aligned $\mathrm{Cu}$ nanowires ${ }^{308}$ were developed using porous alumina as a template. Ni-Pd as a catalyst was supported on porous alumina for hydrogenation and oxidation reactions. ${ }^{309}$ Nanoporous anodic alumina is also considered to be an efficient material for the development of biosensors due to the ease of fabrication, tunable properties, optical/electrochemical properties, and excellent stability in aqueous environments. ${ }^{310}$ 


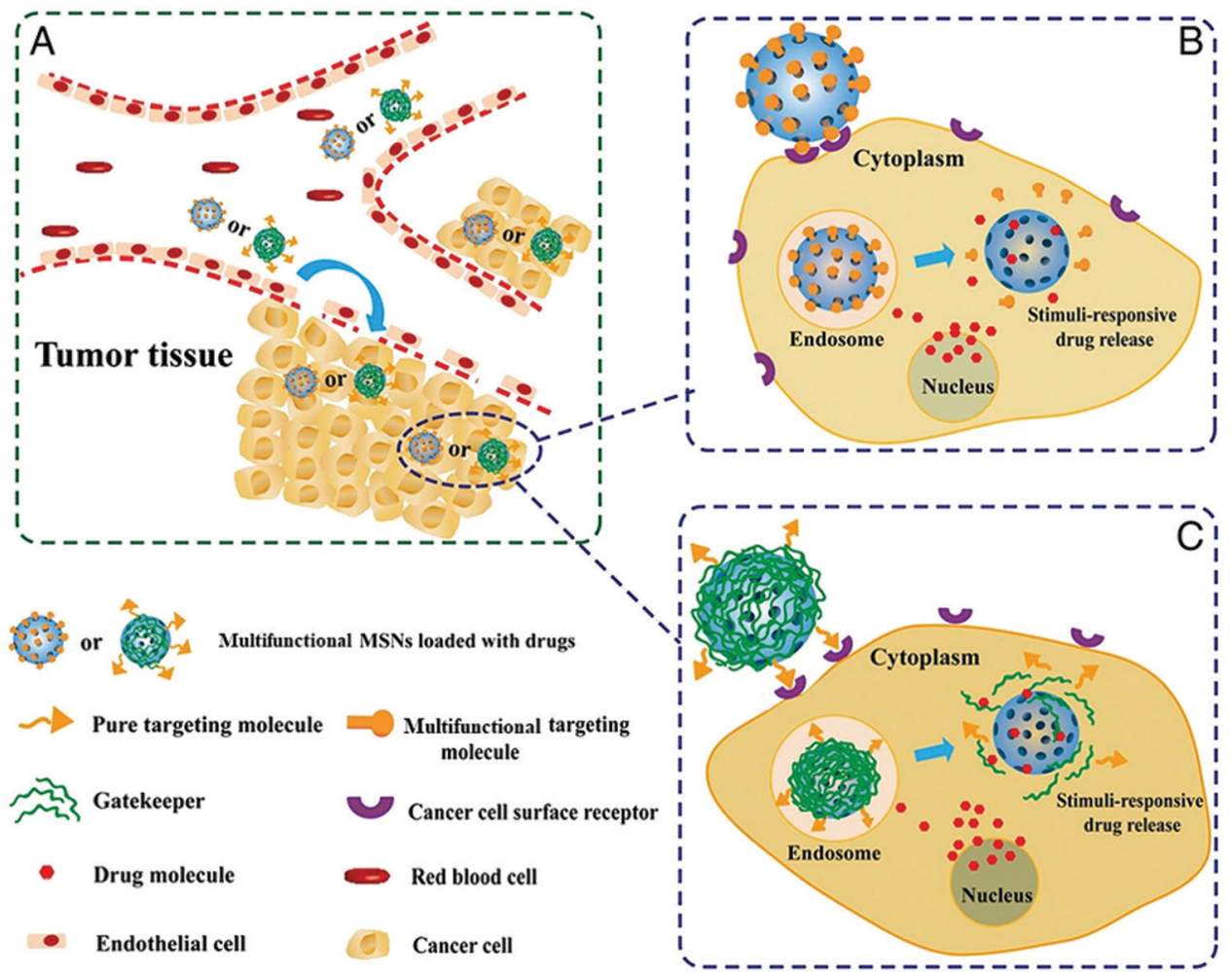

Fig. 27 (A) The in vivo process of an MSN-based controlled and targeted drug-delivery system. Two approaches for multifunctional MSN-based drug delivery systems are to design (B) a multifunctional targeting molecule that acts both as a targeting and capping agent or (C) a stimuli-responsive gatekeeper that is further modified with a target moiety to achieve multifunctional drug delivery. Reprinted with permission from ref. 296. Copyright: (C)2015, Elsevier Inc. All rights reserved.

Nanoporous carbon-based materials are a hot topic in the field of materials chemistry. Nanoporous carbon materials have become ubiquitous choices in the environmental, energy, catalysis, and sensing fields due to their unique morphologies, large pore volumes, controlled porous structures, mechanical, thermal, and chemical stabilities, and high specific surface areas (Fig. 28A). ${ }^{311}$ Nanoporous materials are found to be useful in the treatment of water. The separation of spilled oil and organic pollutants from water has emerged as a significant challenge. ${ }^{312-314}$ The design of materials that can allow the efficient separation of organic, dye, and metal contaminants from water has become a leading environmental research area. ${ }^{315,316}$ Nanoporous carbon can be derived from different natural and synthetic sources. ${ }^{317-319}$ Nanoporous carbon foam can be derived from natural sources, such as flour, pectin, and agar, via table-salt-assisted pyrolysis. The agar-derived nanoporous carbon foam showed high absorption capacities, a maximum of 202 times its own weight, for oil and organic solvents. Air filtration paper developed from carbon nanoporous materials and non-woven fabrics has shown a filtration efficiency of greater than 99\% (Fig. 28B). ${ }^{320}$ Nanoporous carbon can also be produced from other porous frameworks, such as metal-organic frameworks. MOF- and COF-based materials are promising precursors for nanoporous carbon-based materials. The direct carbonization of amino-functionalized aluminum terephthalate metal-organic frameworks has produced nitrogen-doped nanoporous carbon that shows an adequate removal capacity of $98.5 \%$ for methyl orange under the optimum conditions. ${ }^{321} \mathrm{Fe}_{3} \mathrm{O}_{4} /$ nanoporous carbon was also produced with Fe salts as a magnetic precursor and MOF-5 as a carbon precursor for removing the organic dye methylene blue (MB) from aqueous solutions. ${ }^{322}$ The mesoporous carbon removal efficiency could be further enhanced via modifying or functionalizing the surface with various materials. Unmodified mesoporous carbon has shown a mercury removal efficiency of $54.5 \%$. This efficiency can be substantially improved to $81.6 \%$ and $94 \%$ upon modification with the anionic surfactant sodium dodecyl sulfate and cationic surfactant cetyltrimethyl ammonium bromide (CTAB), respectively. ${ }^{323}$

Ordered nanoporous carbon, CNTs, and fullerenes are extensively applied for energy and environmental applications. The complicated synthesis routes required for fullerenes and CNTs have slowed down the full exploitation of their potential for highly demanding applications. In comparison, the synthesis of highly ordered nanoporous carbon is facile, and the properties of ordered nanoporous carbon are also appealing for energy and environmental applications. ${ }^{311} \mathrm{CO}_{2}$ is a greenhouse gas, and its sustainable conversion into value-added products has become the subject of extensive research. A nitrogen-doped nanoporous-carbon/ carbon-nanotube composite membrane is a high-performance gas-diffusion electrode applied for the electrocatalytic conversion of $\mathrm{CO}_{2}$ into formate. A faradaic efficiency of $81 \%$ was found for the production of formate. ${ }^{324}$ Nanoporous carbon materials modified with the non-precious elements $\mathrm{P}, \mathrm{S}, \mathrm{N}$, and $\mathrm{B}$ have 


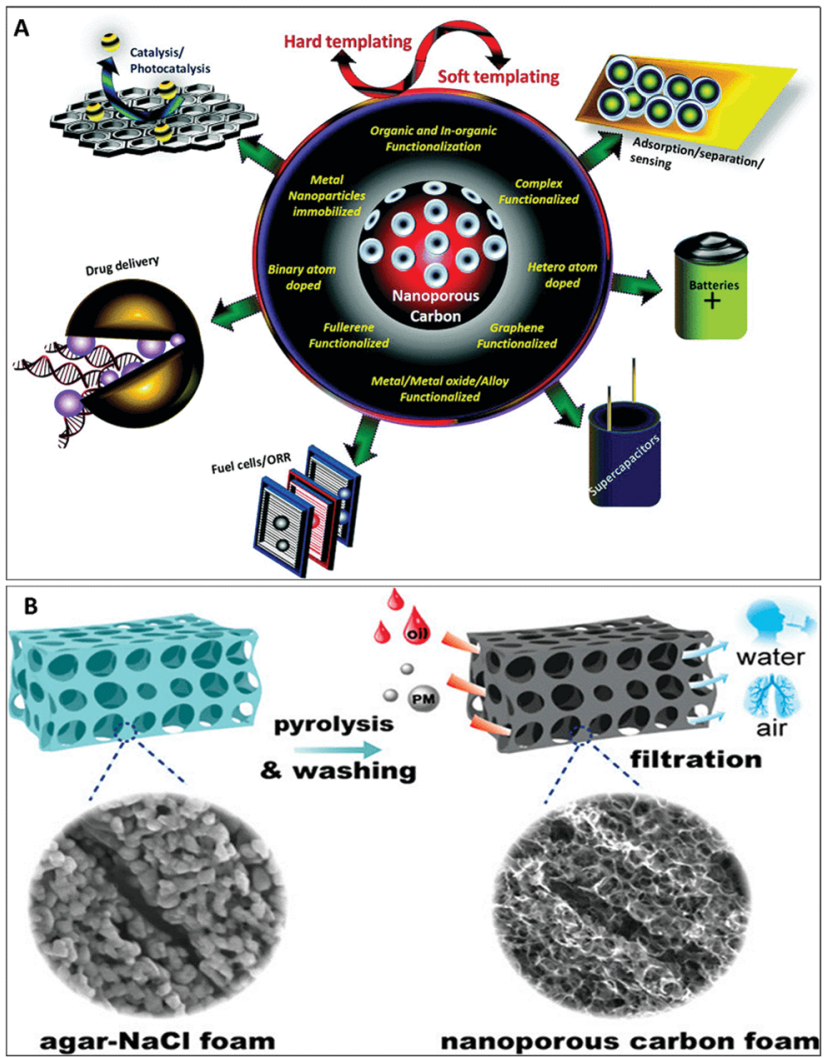

Fig. 28 (A) A schematic illustration of the synthesis, functionalization, and applications of micro- and mesoporous carbon. Reproduced from ref. 311 with permission from The Royal Society of Chemistry. (B) The development of nanoporous carbon foam with high efficiency for oil/organic solvent adsorption. Reprinted with permission from ref. 320. Copyright: (C)2020, American Chemical Society.

emerged as efficient electrode materials for use in the oxygen evolution reaction (OER), hydrogen evolution reaction (HER), oxygen reduction reaction (ORR), batteries, and fuel cells. . $^{311,325-327}$

Nanoporous polymers, including nanoporous coordination polymers and crystalline nanoporous polymers, have emerged as impressive nanoporous materials. ${ }^{328}$ Nanoporous polymers have many applications, and these materials are extensively being evaluated for gas separation and gas storage. The great interest in these applications arises from the presence of pores providing an exceptionally high Brunauer-Emmett-Teller (BET) surface area. Recently, new classes of metal organic framework and covalent organic framework porous materials have been reported that have shown exceptionally high and unprecedented surface areas. For instance, in 2010, a MOF was reported with a surface area of $6143 \mathrm{~m}^{2} \mathrm{~g}^{-1} ;^{329}$ in 2012, a MOF was reported with a surface area greater than $7000 \mathrm{~m}^{2} \mathrm{~g}^{-1} ; 330$ and in 2018, a MOF (DUT-60) was reported with a record surface area of $7836 \mathrm{~m}^{2} \mathrm{~g}^{-1} \cdot{ }^{331}$ Mesoporous DUT-60 has also shown a high free volume of $90.3 \%$ with a density of $0.187 \mathrm{~g} \mathrm{~cm}^{-3} .331$

Due to their exceptionally high surface areas and porous networks, these MOFs and COFs are ideal for gas storage. Air separation and post-combustion $\mathrm{CO}_{2}$ capture have become integral parts of mainstream industries related to the energy sector in order to avoid substantial economic penalties. Due to the inefficiencies of available technology and the critical importance of this area, earnest efforts are being made to design gasselective porous materials for the selective adsorption of desired gases. Nanoporous MOF- and COF-based materials can significantly capture $\mathrm{CO}_{2}$ and help reach zero or minimum $\mathrm{CO}_{2}$ emission levels. For instance, nanoporous fluorinated metal-organic frameworks have shown the selective adsorption of $\mathrm{CO}_{2}$ over $\mathrm{H}_{2}$ and $\mathrm{CH}_{4} \cdot{ }^{332}$ Hasmukh A. Patel et al. developed $\mathrm{N}_{2}$-phobic nanoporous covalent organic polymers for the selective adsorption of $\mathrm{CO}_{2}$ over $\mathrm{N}_{2}$. The azo groups in the framework rejected $\mathrm{N}_{2}$, leading to $\mathrm{CO}_{2}$ selectivity. ${ }^{333}$ Nanoporous polymers that are superhydrophobic in nature can also be used for volatile organic compounds and organic contaminants. ${ }^{334}$ Nanoporous polymers, due to the presence of a porous network, have been considered as highly suitable materials for catalyst supports. Furthermore, organocatalytic functional groups can be introduced pre-synthetically and post-synthetically into solid catalysts. ${ }^{335}$

Nanoporous polymeric materials are amazingly heading towards being extremely lightweight with exceptionally high surface areas. These high surface areas and the fine-tuning of the nanopores has made these nanoporous materials, specifically MOFs and zeolites, ideal support materials for encapsulating ultrasmall metal nanoparticles inside void spaces to produce nanocatalysts with exceptionally high efficiencies. ${ }^{336}$ In the coming years, more exponential growth of nanoporous materials is expected in the energy, targeted drug delivery, catalysis, and water treatment fields.

\subsection{Ultrathin two-dimensional nanomaterials beyond graphene}

Based on their dimensions, as discussed, nanomaterials are classified as zero-dimensional, one-dimensional, two-dimensional, or three-dimensional (0D, 1D, 2D, or 3D, respectively). Materials with nanoscopic dimensions have great technological importance. ${ }^{337,338}$ Among the various systems, 2D nanomaterials represent an emerging class of nanomaterial because of their unique morphological arrangements of sheet-like structures with large lateral size $(\geq 100 \mathrm{~nm})$ and few-atom thickness (typically $<5 \mathrm{~nm}$ ). ${ }^{339}$ After discovering mechanically exfoliated graphene from graphite, ${ }^{340}$ ultrathin 2D nanomaterials have attained great attention for many sustainable-energy applications utilizing their remarkable physiochemical and electronic properties. The features of $2 \mathrm{D}$ nanomaterials can confine electrons into the ultrathin region, making them transportable within the plane. Due to strong in-plane covalent bonding behavior, they possess robust mechanical strength, flexibility, and optical transparency. ${ }^{341}$ The unique properties of ultrathin $2 \mathrm{D}$ nanomaterials can be easily enhanced based on potential fine-tuned substructures. Thus, the search for two-dimensional materials with great flexibility, different compositions, and functional diversity has become a hot area of advanced research. In this section, we summarize several recently developed graphene-like ultrathin 2D nanomaterials that rival graphene materials. We also detail the atomic arrangements in 
these 2D nanomaterials, of either crystalline or amorphous form, that allow various applications.

5.3.1. Silicene. Silicene, a $2 \mathrm{D}$ silicon ( $\mathrm{Si}$ ) allotrope that is one atomic layer thick with an exclusive low-buckled structure, has attracted strong theoretical attention in recent years due to its many superior properties, which it shares with graphene. The first report on this Si-based graphene-like sheet was made in 1994 by Takeda and Shiraishi. ${ }^{342}$ Later, in 2007, Guzman-Verri et al. reinvestigated the similarities between the structural properties of this novel $\mathrm{Si}$ nanomaterial and graphene, and dubbed it "silicene". ${ }^{343}$ Unlike C atoms in graphene, Si atoms are not exactly in the same plane when they are in silicene sheets, and they are speculated to show $\mathrm{sp}^{3} / \mathrm{sp}^{2}$-like hybridization, resulting in a buckled honeycomb atomic arrangement. Furthermore, according to DFT calculation data reported by Cahangirov et al., ${ }^{344}$ the typical Si-Si distance within this honeycomb structure of robust silicene is $0.225 \mathrm{~nm}$, with an ambipolar system indicated by the electronic density of states. First-principles calculations relating to silicene revealed that the electronic structure of silicene is identical to that of graphene, demonstrating semi-metallic characteristics. ${ }^{345,346}$ In addition, the enhanced physical properties, including the quantum spin Hall effect, ${ }^{347}$ chiral superconductivity, ${ }^{348}$ and giant magnetoresistance, ${ }^{349}$ made silicene attractive with widespread potential applications in electronic devices, chemical sensors, hydrogen storage, and electrode materials for lithium-ion batteries. ${ }^{350}$

However, from a material synthesis standpoint, a graphitelike layered form of $\mathrm{Si}$ does not exist in nature and there is no conventional exfoliation process that can generate 2D silicene, although single-walled ${ }^{351}$ and multi-walled ${ }^{352}$ silicon nanotubes and even monolayers of silicon have been synthesized via exfoliation methods. ${ }^{353}$ Forming honeycomb Si nanostructures on substrates like $\mathrm{Ag}(001)$ and $\mathrm{Ag}(110)$ via molecular beam deposition, so-called "epitaxial growth", was then proposed as a method for the architectural design of silicene sheets. ${ }^{354-356}$ The successful synthesis of a silicene monolayer was first achieved on $\mathrm{Ag}(111)$ and $\mathrm{ZrB}_{2}(0001)$ substrates in $2012 ;^{357,358}$ later, various demonstrations were made using $\operatorname{Ir}(111), \mathrm{ZrB}_{2}(001), \mathrm{ZrC}(111)$, and $\mathrm{MoS}_{2}$ surfaces as the silicene growth substrates. ${ }^{359-361}$ Despite various extensive studies to date involving the "epitaxial growth" of silicene on different substrates and investigations of the electronic properties, ${ }^{357,362-364}$ the limited nanometer size, difficulties relating to substrate removal, and air stability issues have substantially impeded the practical applications of silicene. Bearing in mind all these known difficulties, Akinwande and co-workers recently reported a growthtransfer-fabrication process for novel silicene-based field-effect transistor development that involved silicene-encapsulated delamination with native electrodes. ${ }^{365}$ An etch-back approach was used to define source/drain contacts in Ag film. Without causing any damage to the silicene, a novel potassium-iodide-based iodine-containing solution was used to etch Ag, avoiding rapid oxidation, unlike other commonly used Ag etchants. The results demonstrated that this was the first proof-of-concept study confirming the Dirac-like ambipolar charge transport predictions made about silicene devices. Comparative studies with a graphene system, the low residual carrier density, and the high gate modulation suggested the opening of a small bandgap in the experimental devices, proving that silicene can be considered a viable $2 \mathrm{D}$ nanomaterial beyond graphene.

Nonetheless, the synthesis of silicene on a large-scale is greatly limited, as "epitaxial growth" is the only promising method for obtaining high-quality silicene, and this presents an enduring challenge in relation to silicene research and development. $\mathrm{Xu}$ and co-workers recently introduced liquid oxidation and the exfoliation of $\mathrm{CaSi}_{2}$ as a means for the first scalable preparation of high-quality silicene nanosheets. ${ }^{366}$ This new synthetic strategy successfully induced the exfoliation of stacked silicene layers via the mild oxidation of the $\left(\mathrm{Si}^{2 n}\right)^{2 n}$ layers in $\mathrm{CaSi}_{2}$ into neutral $\mathrm{Si}^{2 n}$ layers without damage to the pristine silicene structure (Fig. 29). The selective oxidation of pristine $\mathrm{CaSi}_{2}$ into free-standing silicene sheets without any damage to the original $\mathrm{Si}$ framework was carried out via exfoliation in the presence of $\mathrm{I}_{2}$ in acetonitrile solvent. Furthermore, the obtained silicene sheets yielded ultrathin monolayers or layers with few-layer thickness and exhibited excellent crystallinity. This 2D silicene nanosheet material was extensively explored as a novel anode, which was unlike previously developed silicon-based anodes for lithium-ion batteries. It displayed a theoretical capacity of $721 \mathrm{~mA} \mathrm{~h} \mathrm{~g}^{-1}$ at $0.1 \mathrm{~A} \mathrm{~g}^{-1}$ and superior cycling stability of 1800 cycles. Overall, during the last decade, silicene has been widely accepted as an ideal 2D material with many fascinating properties, suggesting great promise for a future beyond graphene.

5.3.2. MXenes. Given the essential utility of $2 \mathrm{D}$ nanomaterials, various graphene-like 2D nanomaterials have attracted extensive attention in recent years due to their unprecedented physical and

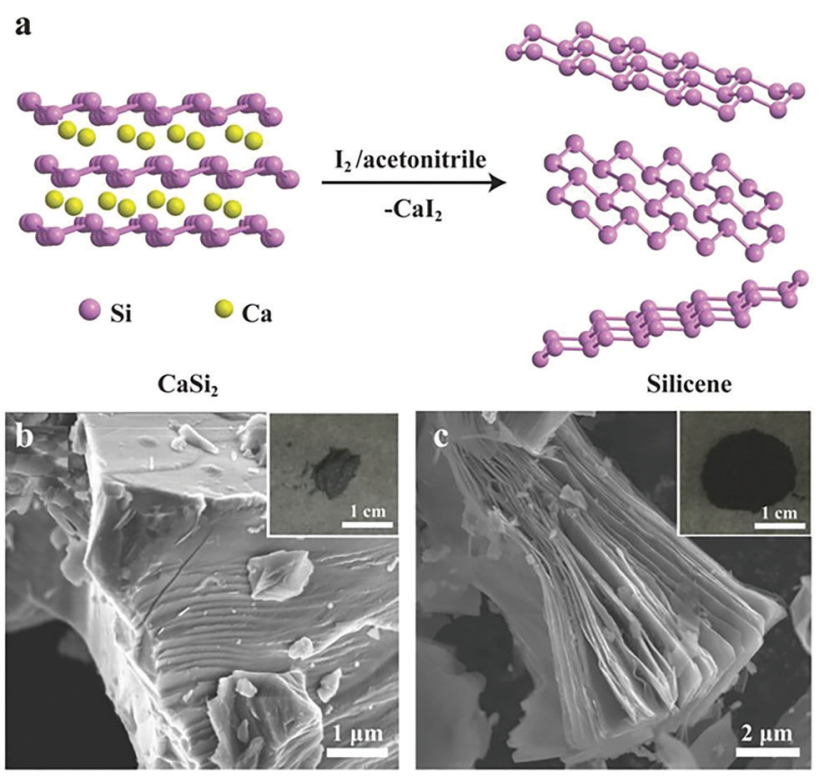

Fig. 29 (a) A schematic illustration of the synthesis of silicene from $\mathrm{CaSi}_{2}$ via liquid oxidation and exfoliation. SEM images of pristine bulk $\mathrm{CaSi}_{2}$ (b) and the as-reacted product (c), with insets showing photographs. Reprinted with permission from ref. 366. Copyright: (C)2018, WILEY-VCH Verlag $\mathrm{GmbH} \& \mathrm{Co}$. KGaA, Weinheim. 
chemical properties. Most recently, the group of 2-D materials has been expanded enormously by the addition of early transition metal carbides, nitrides, and carbonitrides from MAX-phases, which are known as MXenes. ${ }^{367}$ With a resemblance to graphene, MXenes share a similar lateral size, varying from hundreds of nanometers to several micrometers, including a theoretical thickness of $0.98 \mathrm{~nm}$ (single layer), together with outstanding physical and chemical properties. ${ }^{368}$ In general, MXenes are composed of transition metal carbides, nitrides, or carbonitrides having few-atom-thick layers. The conventional form of MXenes is $\mathrm{M}_{n+1} \mathrm{X}_{n} \mathrm{~T}_{x}(n=1,2$, or 3$)$, where $\mathrm{M}$ denotes a transition metal element, and $\mathrm{X}$ and $\mathrm{T}_{x}$ represent carbon and/or nitrogen atoms and other surface functional groups $(-\mathrm{O},-\mathrm{OH}$, or $-\mathrm{F})$, respectively. ${ }^{369}$ In other words, MXenes are synthesized from layered MAX-phase precursors $\left(\mathrm{M}_{n+1} \mathrm{X}_{n}\right)$ consisting of modulated terminating groups (i.e., $-\mathrm{O},-\mathrm{OH}$, or $-\mathrm{F}$ ) via a controlled etching process. This etching step can be further modified to produce fine-tuned MXenes with versatile physical properties, utilizing the practical applicability of the material designability. The first report on MXenes $\left(\mathrm{Ti}_{3} \mathrm{C}_{2} \mathrm{~T}_{x}\right)$ involved the exfoliation of MAX-phases as a substitute for graphene in supercapacitors by Naguib, Barsoum, and Gogotsi from Drexel University, USA, in 2011. ${ }^{370}$ Since then, more than 19 forms of MXenes have subsequently been developed, and various predictions relating to MXenes structures have also been made.

Like other 2D materials, MXenes exhibit crystal geometry with a hexagonal close-packed structure based on the equivalent MAX-phase precursor, and the close-packed structure is formed from $\mathrm{M}$ atoms with $\mathrm{X}$ atoms occupying octahedral sites. ${ }^{371}$ According to the formula, there are three representative structures of MXenes: $\mathrm{M}_{2} \mathrm{XT}_{x}, \mathrm{M}_{3} \mathrm{X} 2 \mathrm{~T}_{x}$, and $\mathrm{M}_{4} \mathrm{X} 3 \mathrm{~T}_{x}$. In these combinations, $\mathrm{X}$ atoms are formed with $n$ layers, whereas $\mathrm{M}$ atoms have $n+1$ layers (Fig. 30). ${ }^{372}$ Apart from graphene, MXenes are considered the most dynamic developing material, and they have incredible innovation potential amongst typical 2D nanomaterials because of their remarkable properties, such as hydrophilicity, conductivity, considerable adsorption abilities, and catalytic activity. These vital properties of MXenes suggest their use for various potential applications, including in the photocatalysis, electrocatalysis, ${ }^{373,374}$ energy, ${ }^{375}$ membrane-based separation, ${ }^{376,377}$ and biological therapy ${ }^{378}$ fields. In this section, we focus on describing new developments relating to MXenes that are utilized for electrocatalytic and energy storage applications, competing as alternatives to graphene materials.

Interestingly, due to the presence of abundant terminal groups, mainly $-\mathrm{O},-\mathrm{OH}$, and $-\mathrm{F}$, and their modifying nature, MXenes can exhibit outstanding hydrophilic properties and high conductivity and charge carrier mobility, making them a very attractive material for various electrocatalytic applications, such as the hydrogen evolution reaction, oxygen evolution reaction, oxygen reduction reaction, nitrogen reduction reaction, and $\mathrm{CO}_{2}$ reduction reaction. To further increase their electrocatalytic activities, recent works involving MXenes have included incorporation with CNTs, ${ }^{379}$ g- $_{3} \mathrm{~N}_{4},{ }^{380} \mathrm{FeNi}-\mathrm{LDH},{ }^{381}$ NiFeCo-LDH, ${ }^{382}$ and metal-organic frameworks. ${ }^{383}$

Cho and co-workers designed and developed MXene-TiO $_{2}$ 2D nanosheets via the surface oxidation of MXene with defectfree control. These $\mathrm{MXene}-\mathrm{TiO}_{2} 2 \mathrm{D}$ nanosheets were successfully implemented in nano-floating-gate transistor memory (NFGTM) providing a floating gate (i.e., multilayer MXene) and tunneling dielectric (i.e., the $\mathrm{TiO}_{2}$ layer). A process of oxidation in water further represented a cost-effective and environmentally benign method, as depicted in Fig. 31. The MXene NFGTM with an optimal oxidation process displayed exceptional nonvolatile memory features, having a great memory window, high programming/erasing current ratio, long term retention, and high durability. ${ }^{384}$

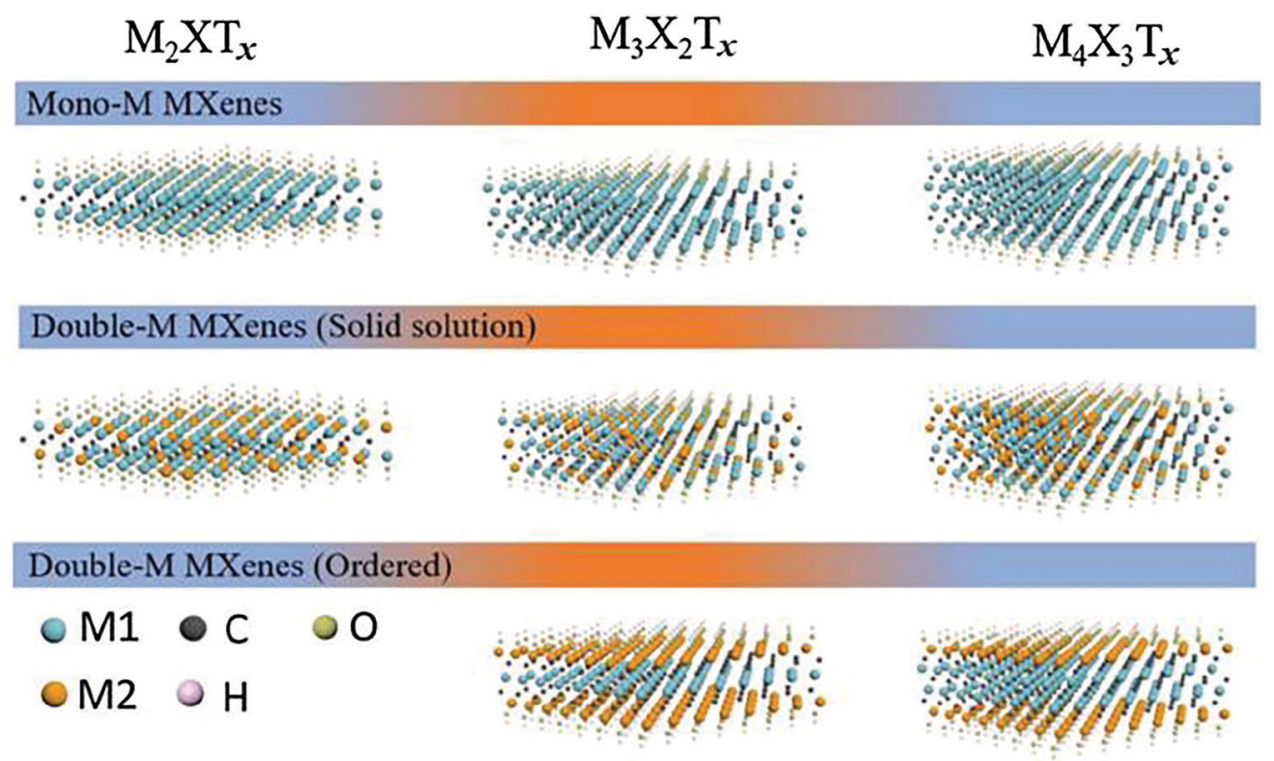

Fig. 30 The structures of MXenes with different formulas $\left(M_{2} X T_{x}, M_{3} X_{2} T_{x}\right.$, and $\left.M_{4} X_{3} T_{x}\right)$ and their compositions (mono-M MXenes and double-M MXenes). Reprinted with permission from ref. 372. Copyright: (C)2019, WILEY-VCH Verlag GmbH \& Co. KGaA, Weinheim. 


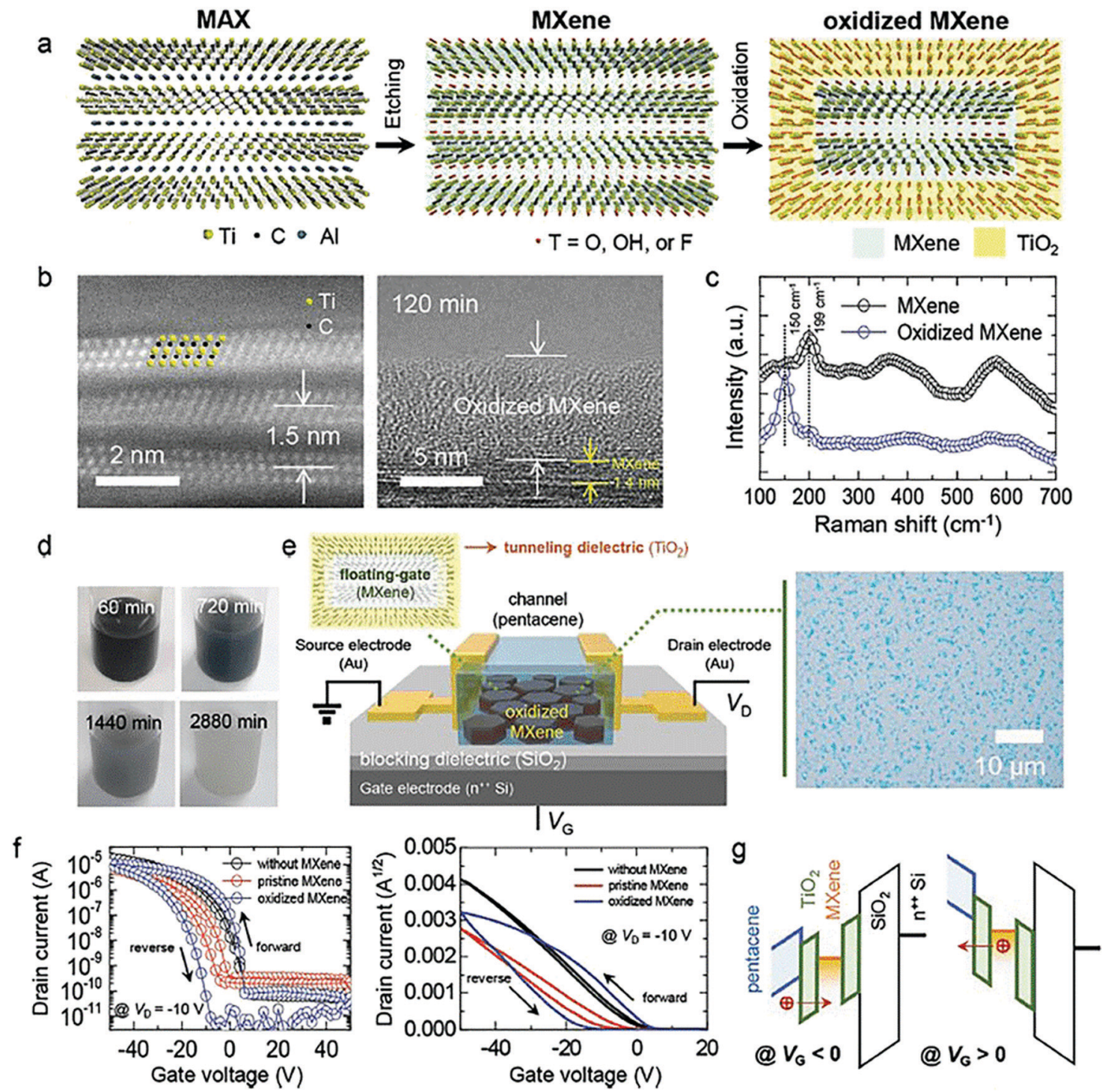

Fig. 31 (a) A schematic diagram of the synthesis of $2 \mathrm{D} \mathrm{Ti}{ }_{3} \mathrm{C}_{2} \mathrm{~T}_{x} M X$ Xene and its controlled oxidation. (b) Cross-sectional STEM images of pristine MXene and oxidized MXene. (c) Raman spectra of pristine MXene and oxidized MXene. (d) Photographic images of MXene solution at different stages of oxidation. (e) A schematic illustration of MXene NFGTM. The right panel shows an optical microscopy (OM) image of the oxidized MXene on the $\mathrm{SiO}_{2}$ blocking dielectric layer. (f) The transfer characteristics of transistors with different hysteresis behavior. (g) A schematic diagram of the energy bands of MXene NFGTM corresponding to hole trapping and detrapping in the MXene floating gate. Reprinted with permission from ref. 384. Copyright: (C)2020, WILEY-VCH Verlag GmbH \& Co. KGaA, Weinheim.

5.3.3. 2D materials from group 15: the importance of arsenene and antimonene. Over the past few years, 2D nanomaterials composed of group 15 elements have received attention due to their similarity in behavior to graphene-related materials and their suitability for forming mono-elemental 2D layered nanomaterials with potential for a variety of applications, such as being used in optoelectronics and electronics, ${ }^{385,386}$ plasmonics, ${ }^{387}$ and energy-storage devices. ${ }^{388,389}$ 2D materials made of group 15 elements, commonly known as pnictogens, comprise one-atom-thick 2D structures with monolayers possessing a broad range of band gaps, for example, phosphorene from black phosphorus, and sharing similar interesting properties with graphene materials, such as electronic and vibrational properties.
There have been some exciting reports on 2D materials from the pnictogen family, particularly phosphorene. Recently, more attention has also been given to the remaining group 15 elements, ${ }^{390}$ with the novel $2 \mathrm{D}$ materials arsenene, antimonene, and bismuthene being obtained from the key elements arsenic, antimony, and bismuth, respectively. It is reported that 2D monolayers of group 15 elements, including phosphorene allotropes, have five distinct honeycomb $(\alpha, \beta, \gamma, \delta$, and $\varepsilon)$ and four distinct non-honeycomb $(\zeta, \eta, \theta$, and 1$)$ structures, as depicted in Fig. 32. Dissimilar crystal orientations were found for single-layered $\mathrm{As}, \mathrm{Sb}$, and $\mathrm{Bi}$. Zeng and co-workers also reported comprehensive density functional theory (DFT) computations that proved the energetic stability and broad-range 
$\alpha$

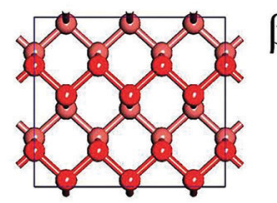
$\beta$
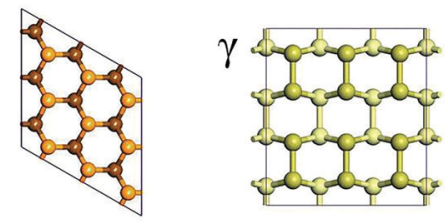

$\delta$

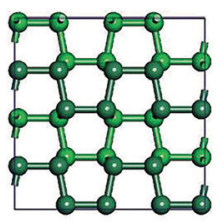

$\varepsilon$

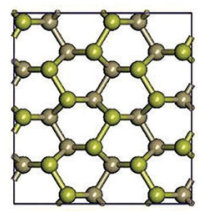

$\zeta$

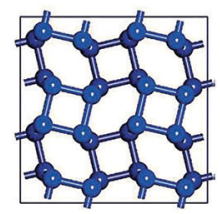

$\eta$

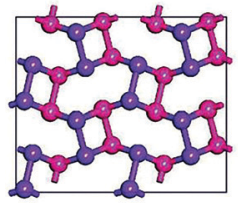

1

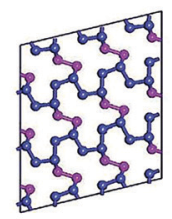

$\theta$

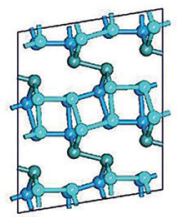

Fig. 32 Top views of relaxed group 15 monolayer allotropes with five typical honeycomb structures $(\alpha, \beta, \gamma, \delta$, and $\varepsilon)$ and four typical non-honeycomb structures $(\zeta, \eta, \theta$, and 1$)$. Reprinted with permission from ref. 391. Copyright (C)2016, WILEY-VCH Verlag GmbH \& Co. KGaA, Weinheim.

application of these materials in $2 \mathrm{D}$ semiconductors. ${ }^{391}$ Previously, following theoretical predictions, $\mathrm{Wu}$ and co-workers successfully demonstrated that $\alpha$-phosphorene showed lowest energy configurations in both honeycomb and non-honeycomb nanosheets. ${ }^{392}$ In contrast, Zeng and co-workers proved that the buckled forms of $2 \mathrm{D}$ sheets of $\mathrm{As}, \mathrm{Sb}$, and $\mathrm{Bi}$ allotropes are the most stable structures, particularly their $\beta$ phases. ${ }^{391}$

Among monolayer group 15 family materials, 2D sheets of arsenic (As) and antimony ( $\mathrm{Sb}$ ) have gained considerable attention from researchers. ${ }^{393,394}$ Studies have shown that $\mathrm{As}$ and $\mathrm{Sb}$ exhibit better stability than black phosphorus; they are highly stable at room temperature and less reactive to air, likely inhibiting the oxidization process. ${ }^{395-398}$ Nevertheless, it has been demonstrated that the oxidation process is perhaps favorable for fine-tuning the electronic properties; increases in the indirect band gaps ranging from 0 to a maximum of $2.49 \mathrm{eV}$ are found in free-standing arsenene and antimonene semiconductors. ${ }^{399-403}$ Simultaneously, arsenene and antimonene can also be transformed into semiconductors with direct band gaps. These two 2D nanosheets can be used to design mechanical sensors, moving beyond common electronic and optoelectronic applications. These two extraordinary $2 \mathrm{D}$ nanosheets have been studied for their structural-property relationships via first-principles methods. ${ }^{403-405}$

Continuing the characterization and structural property studies of arsenene carried out by $\mathrm{Kamal}^{404}$ et al. and Zhang ${ }^{403}$ et al., Anurag Srivastava and co-workers analyzed applications of arsenene to explore the possibility of improving sensor devices that can be utilized to detect ammonia $\left(\mathrm{NH}_{3}\right)$ and nitrogen dioxide $\left(\mathrm{NO}_{2}\right)$ molecules. ${ }^{406,407}$ They investigated the affinities of $\mathrm{NH}_{3}$ and $\mathrm{NO}_{2}$ molecules for pristine arsenene sheets, examining the binding energies, bonding distances, density distributions, and current-voltage features. The results showed that arsenene 2D sheets are highly durable, with significant electronic charge transfer. They also considered germanium-doped arsenene and characterized the 2D lattice based on molecular affinity relationships with respect to the dopant.

However, the incorporation of any dopants into 2D nanomaterials not only results in experimental difficulty but it also lowers the stability of 2D materials. ${ }^{408}$ Recently, Dameng Liu and co-workers reported the electronic structures, focusing on band structures, band offsets, and intrinsic defect properties, of few-layer arsenic and antimony. ${ }^{409}$ The spontaneous oxide passivation layer that is formed naturally on pristine antimonene provides excellent stability. ${ }^{410}$ Very recently, Stefan Wolff and co-workers conducted DFT calculations on various single or few-layer antimony oxide structures to describe the stoichiometry and bonding type. Interestingly, the samples exhibited various structural stabilities and electronic properties with a wide range of direct and indirect band gaps. Showing band gaps between 2.0 and $4.9 \mathrm{eV}$, these $2 \mathrm{D}$ layers of antimonene exhibited the potential to be used as insulators or semiconductors. ${ }^{411}$ The same group also analyzed Raman spectra and discussed identifying the predicted antimonene oxide structures experimentally. The enduring task of exploring the utility of antimonene has boosted recent research interest in 2D nanomaterials due to the broad range of potential applications, such as their use in electrochemical sensors, ${ }^{412,413}$ stable organic solar cells, ${ }^{414}$ and supercapacitors ${ }^{415}$ to name a few.

5.3.4. Two-dimensional metal-organic framework nanosheets (2D-MOFs). Nanosheets composed of two-dimensional metalorganic frameworks have emerged as a new class of 2D material with distinctive characteristic features, such as ultrathin thicknesses, tailorable molecular porous structures, and high surfaceto-volume ratios. Due to their exceptional physical and chemical properties, these novel 2D-MOF nanosheets produce highperformance 2D materials with wide application prospects, including for electrochemical, sensing platform, and membranebased gas separation uses, with many more applications possible. As a result, over the past few years there have been significant advances in the design and synthesis of 2D-MOF nanosheets for many applications. Common synthetic routes for preparing pristine 2D-MOF nanosheets usually involve top-down methods and bottom-up methods. In general, top-down methods cover the strategy of the single or few-layer exfoliation of MOF nanosheets through various techniques, including ultrasonicassisted exfoliation, ${ }^{416}$ mechanically forced exfoliation, ${ }^{417} \mathrm{Li}$-ion intercalation-based exfoliation, ${ }^{418,419}$ and chemically derived exfoliation. ${ }^{420}$ In bottom-up methods, the direct synthesis is similar to common MOF preparation methods from metal ions and organic linkers. Bottom-up strategies mainly include interfacial synthesis, ${ }^{421}$ modulated synthesis, ${ }^{422}$ three-layer synthesis, ${ }^{423}$ and surfactant-mediated synthesis. ${ }^{424} \mathrm{~A}$ handful of review articles about 2D-MOFs from a synthetic perspective discuss the challenging issues of the large-scale preparation and direct applications of pristine 2D-MOF nanosheets. However, many recent works have 
reported outstanding functional 2D-MOF nanomaterials with a wide range of applications. This section aims to focus more on the potential applications of 2D-MOF nanosheets and address recent progress in this area.

5.3.4.1. Electrochemical applications. The tunable structures and tailorable features of 2D-MOFs have allowed high performance for many electrochemical applications. Most importantly, the featured high specific surface areas of 2D-MOFs have gained much attention for use in supercapacitors. For example, a recent work by Zheng and co-workers proved that cobalt-based 2D-MOFs synthesized using polyvinylpyrrolidone as a surfactant under mild solvothermal conditions could show higher specific capacitance due to the large surrounding area (Fig. 33). The developed 2D Co-MOF nanosheets have shown a maximum specific capacitance of $1159 \mathrm{~F} \mathrm{~g}^{-1}$ at a current density of $0.5 \mathrm{~A} \mathrm{~g}^{-1} .^{425}$ Similarly, Wang and his colleagues developed ultrathin nanosheets from NiCo-MOFs with thicknesses ranging from 1.74-3.87 $\mathrm{nm}$ and these were compared with pristine $\mathrm{Ni}$ MOFs and Co-MOFs obtained following the same synthetic protocol. The obtained cyclic voltammetry curves revealed that the NiCo-MOF-nanosheet-based electrode yielded a larger CV area than the Ni-MOF and Co-MOF electrodes. ${ }^{426}$

The 2D MOF nanosheets are also evaluated for the development of high-performance power-storage devices. For example,
Li et al. $^{427}$ recently reported two novel Mn-2D MOFs and Ni-2D MOFs as anode materials for rechargeable lithium batteries. The Mn-based ultrathin metal-organic-framework nanosheets, due to thinner nanosheets, a higher specific surface area, and smaller metal ion radius, had structural advantages over Ni-based ultrathin metal-organic-framework nanosheets. Due to these features, the Mn-based ultrathin metal-organicframework nanosheets displayed a high reversible capacity of $1187 \mathrm{~mA} \mathrm{~h} \mathrm{~g}^{-1}$ at $100 \mathrm{~mA} \mathrm{~g}^{-1}$ for 100 cycles and a rate capability of $701 \mathrm{~mA} \mathrm{~h} \mathrm{~g}^{-1}$ even at $2 \mathrm{~A} \mathrm{~g}^{-1}$.

The expensive metal oxides utilized in the catalytic process can be replaced in due course by 2D-MOF-based nanosheets with exposed metal sites that impart an adjustable pore structure, ultrathin thickness, a high surface-to-volume atom ratio, and high design flexibility. As a result, 2D-MOFs have extensively been explored for various electrocatalytic applications, including the hydrogen evolution reaction (HER), oxygen evolution reaction (OER), oxygen reduction reaction (ORR), and carbon dioxide reduction reaction $\left(\mathrm{CO}_{2} \mathrm{RR}\right)$. For example, Marinescu et al. ${ }^{428}$ combined cobalt dithiolene species with benzenehexathiol (BHT) and yielded 2D-MOFs capable of acting as electrocatalysts for the HER in water (Fig. 34). In the presence of 2D-MOF sheets, a high current density of $41 \mathrm{~mA} \mathrm{~cm}^{-2}$, at $-0.8 \mathrm{~V} v s$. SHE and a $\mathrm{pH}$ value of 1.3 , is observed. Similarly, Feng et $a .^{429}$ also developed single-layer Ni-based 2D-MOF sheets that are highly effective
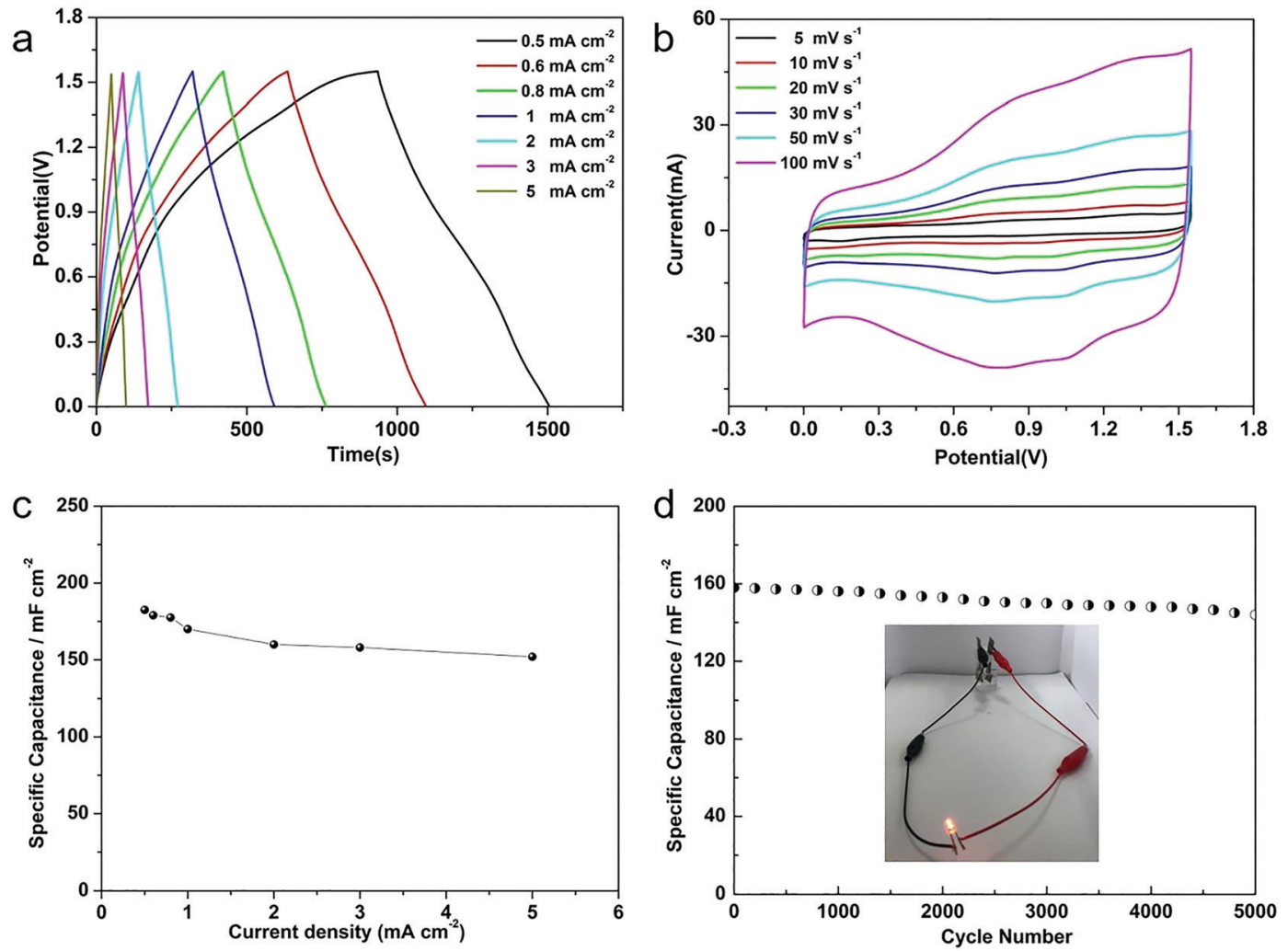

Fig. 33 Electrochemical studies of an as-prepared ultrathin 2D Co-MOF NS//activated carbon aqueous device. (a) The galvanostatic charge-discharge curves at different current densities. (b) The cyclic voltammetry curves at different scan rates. (c) Specific capacitances at different current densities. (d) Charge-discharge cycle testing at a current density of $0.5 \mathrm{~A} \mathrm{~cm}^{-2}$ in $3.0 \mathrm{M} \mathrm{KOH}$ electrolyte (inset: a red LED powered by the aqueous device). Reprinted with permission from ref. 425. Copyright: (C2019, Elsevier B.V. All rights reserved. 


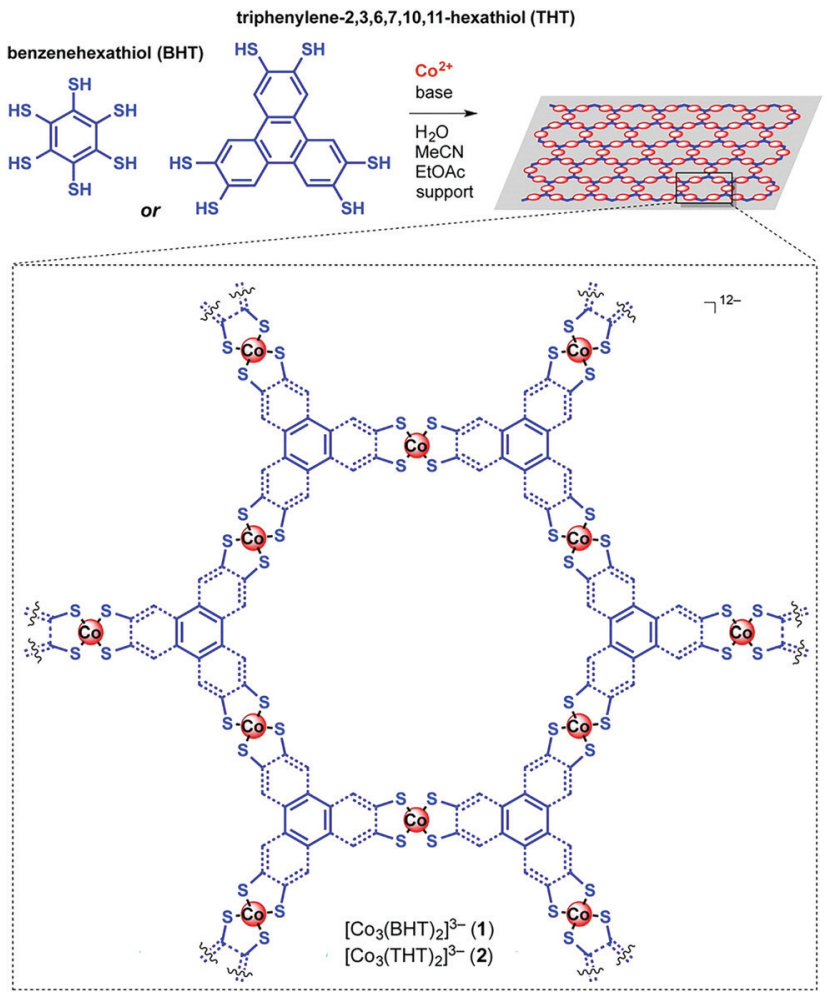

Fig. 34 The synthesis of the cobalt dithiolene films 1 and 2 through a liquid-liquid interfacial reaction. The synthesized films are deposited onto desired supports, generating MOS 1 and 2. Reprinted with permission from ref. 428. Copyright: (C)2015, American Chemical Society.

for electrocatalytic hydrogen evolution. Later, Patra et al. ${ }^{430}$ reported similar 2D sheets from covalent organic frameworks (2D-COFs) as metal-free catalysts for HER applications. 2D-MOFs are also being explored as active catalysts for the OER process. For example, Xu et al. ${ }^{431}$ reported the preparation of 2D Co-MOF sheets using polyvinylpyrrolidone as a surfactant under mild solvothermal conditions. These novel 2D Co-MOFs displayed ultrathin nanosheets with many surface-based metal active sites, improving the overall OER performance.

Interestingly, experimental electrochemical measurement data showed that Co-MOF sheets offer a low overpotential (i.e., $263 \mathrm{mV}$ at $10 \mathrm{~mA} \mathrm{~cm}{ }^{-2}$ ). Similarly, Wang et al. ${ }^{432}$ also reported that double-metal 2D-sheets (2D NiFe MOFs) consisting of a very ultrathin structure with a thickness of $\sim 10 \mathrm{~nm}$ further offer a low overpotential of $260 \mathrm{mV}$ at $10 \mathrm{~mA} \mathrm{~cm}{ }^{-2}$. In other reports, Zhang et al. $^{433}$ successfully performed the OER process with ultrathin 2D-MOF sheets prepared via electrochemical and chemical exfoliation strategies.

Recent work on the catalytic activity of 2D-MOFs has also been reported in relation to the ORR and $\mathrm{CO}_{2} \mathrm{RR}$ because of their layered crystal structures and high-volume modifiable porous structures. For example, Dincă et $a l^{434}$ demonstrated that ultrathin layered conductive sheets of the $2 \mathrm{D}-\mathrm{MOF} \mathrm{Ni}{ }_{3}(\mathrm{HITP})_{2}(\mathrm{HITP}=$ 2,3,6,7,10,11-hexaiminotriphenylene) could actively be utilized as a catalyst in an alkaline medium for the ORR process. These $2 \mathrm{D}$ MOF sheets show high stability while retaining $88 \%$ of the initial current density over $8 \mathrm{~h}$ at $0.77 \mathrm{~V} v s$. RHE. In another report, through fabricating $\mathrm{Co}_{x} \mathrm{Zn}_{2-x}(\text { bim })_{4} 2 \mathrm{D}$-sheets as precursors, Zhao et al. ${ }^{435}$ successfully synthesized cobalt nanodots (Co-NDs) with bimetallic $\mathrm{Co}_{x} \mathrm{Zn}_{2-x}(\mathrm{bim})_{4}$ nanosheets encapsulating few-layer graphene (Co@FLG). For the $\mathrm{CO}_{2} \mathrm{RR}$, a cobalt-porphyrin-containing 2D-MOF was achieved for the selective electrochemical reduction of $\mathrm{CO}_{2}$ to $\mathrm{CO}$ with enhanced stability by Peidong Yang and coworkers. ${ }^{436}$ The results further proved that these thin-film catalysts have the highest selectivity for CO (i.e., 76\%) at $-0.7 \mathrm{~V} v$ s. RHE with the little-to-no substantial decrease in activity over $7 \mathrm{~h}$ at $-0.7 \mathrm{~V}$ vs. RHE, and $16 \mathrm{~mL}$ of $\mathrm{CO}$ was produced. Besides, like many other porous materials, 2D-MOFs were also shown to be a supporting platform for catalytic nanoparticles because of their high specific surface areas and favorable porosity distributions. To this end, an example can be noted from Wang et al. ${ }^{437}$ reporting that fine porous MOF-5 nanosheets can be utilized to immobilize Pd nanoparticles.

5.3.4.2. Sensing platforms. Considering the growing demand for high-performance sensors, the development of practical nanomaterials, particularly 2D-MOFs, has attracted great importance in the fluorescence sensing field to detect small gas molecules, metal ions, organic solvents, and biomolecules. ${ }^{438-440}$ Taking account of their unique physical and chemical properties together with their structural integrity, 2D-MOFs offer auspicious possibilities for sensing applications. So far, 2D-MOFs have been used in the optical and electrochemical sensing platforms and they have been applied to the sensing of DNA, ${ }^{441-443}$ microRNA, ${ }^{419}$ and adenosine. ${ }^{418,444}$ In biosensing applications, for example, Zhang et $a$ l. $^{441}$ reported a simple surfactant-assisted synthetic route for developing ultrathin 2D-MOF sequences with various metals (M-TCPP: $\mathrm{M}=\mathrm{Zn}, \mathrm{Cu}, \mathrm{Cd}$, or Co; TCPP = tetrakis(4-carboxyphenyl)porphyrin) and a sensor that could be effectively employed in the detection of DNA (Fig. 35). The experimental results show that the copper analogue $2 \mathrm{D} \mathrm{Cu}$-TCPP nanosheets performed best at sensing DNA out of four different metals. This counterpart exhibited a detection limit of $20 \times 10^{-12} \mathrm{M}$, and this detection limit is substantially lower than previously reported MOF-based fluorescence assays. The fluorescence quenching nature was later studied for two-color intracellular adenosine imaging in living cells by Xia et $a .^{418}$ Besides being used as quenchers, 2D-MOFs can also be a part of fluorescence-based emitters; fluorescein isothiocyanate (FITC) was covalently conjugated to UiO MOFs and this system exhibited excellent ratiometric $\mathrm{pH}$-sensing and enhanced fluorescence properties, as reported by Lin and co-workers. ${ }^{445}$

5.3.4.3. Membrane-based gas separation. In membrane-based gas separation, the key parameters that characterize the membrane process are permeability (how productive the separation process is) and selectivity (how efficient the separation process is). Due to the nanometer thickness and controlled pore structure distribution, 2D-MOFs have been recognized as a new type of membrane material amongst other gas-separation membranes. To better understand the performance of 2D MOF nanosheets, Yang et $a l .{ }^{446}$ examined the potential applicability of 2D-MOFs via 
a
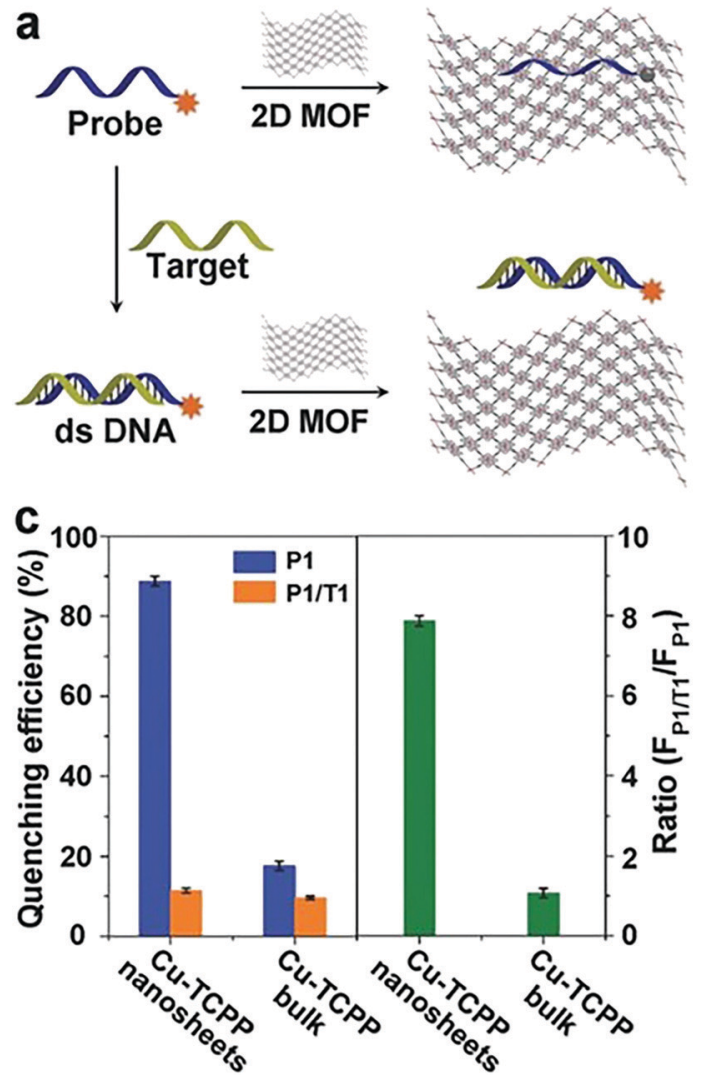
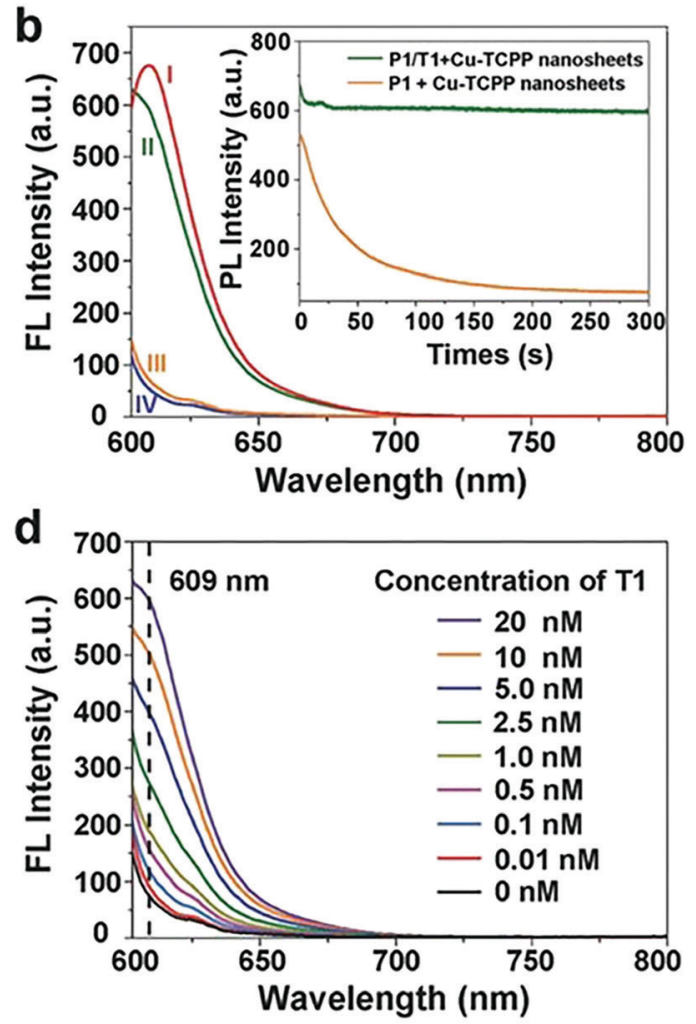

Fig. 35 DNA detection with 2D MOFs (Cu-TCPP, Zn-TCPP(Fe), and Co-TCPP). (a) A schematic illustration of the 2D-MOF-nanosheet-based fluorescence assay for DNA. (b) Fluorescence spectra under different experimental conditions: (I) P1; (II) P1 + T1 + Cu-TCPP nanosheets; (III) P1 + CuTCPP nanosheets; and (IV) Cu-TCPP nanosheets. The concentrations of P1, T1, and Cu-TCPP nanosheets in the final solutions are $2.5 \times 10^{-9} \mathrm{M}, 20 \times 10^{-9} \mathrm{M}$, and $35 \mu \mathrm{g} \mathrm{mL}{ }^{-1}$, respectively. Inset: A kinetics study of the fluorescence changes of the P1 and P1/T1 duplexes in the presence of Cu-TCPP nanosheets; the excitation and emission wavelengths are 588 and $609 \mathrm{~nm}$, respectively. (c) Left: The quenching efficiency ( $\eta$ ) of Cu-TCPP nanosheets and bulk Cu-TCPP MOFs for $\mathrm{P} 1$ and $\mathrm{P} 1 / \mathrm{T} 1$; right: the fluorescence intensity ratio $\left(F_{\mathrm{P} 1 / \mathrm{T} 1} / F_{\mathrm{P} 1}\right)$ values at $609 \mathrm{~nm}$ in the presence of $\mathrm{Cu}-\mathrm{TCPP}$ nanosheets $\left(35 \mu \mathrm{g} \mathrm{mL} \mathrm{L}^{-1}\right)$ or bulk $\mathrm{Cu}-$ TCPP MOFs $\left(35 \mu \mathrm{g} \mathrm{mL}^{-1}\right) . F_{\mathrm{P} 1 / \mathrm{T} 1}$ is the fluorescence intensity of dsDNA (P1/T1) at $609 \mathrm{~nm}$ in the presence of Cu-TCPP nanosheets or bulk Cu-TCPP MOFs. $F_{\mathrm{P} 1}$ is the fluorescence intensity of ssDNA (P1) at $609 \mathrm{~nm}$ in the presence of $\mathrm{Cu}$-TCPP nanosheets or bulk Cu-TCPP MOFs. The concentrations of P1 and T1 in the final solution are $2.5 \times 10^{-9}$ and $20 \times 10^{-9} \mathrm{M}$. (d) Fluorescence spectra of P1 $\left(2.5 \times 10^{-9} \mathrm{M}\right)$ in the presence of T1 at different concentrations in 2D Cu-TCPP nanosheet solution $\left(35 \mu \mathrm{g} \mathrm{mL}^{-1}\right.$ ). Reprinted with permission from ref. 441. Copyright: (C)2015, WILEY-VCH Verlag GmbH \& Co. KGaA, Weinheim.

employing ultrathin molecular sieving membranes for $\mathrm{H}_{2} / \mathrm{CO}_{2}$ separation. A layered poly[ $\left.\mathrm{Zn}_{2}(\text { benzimidazole })_{4}\right] \mathrm{MOF}$, denoted as $\mathrm{Zn}_{2}(\text { bim })_{4}$, with a sheet thickness approximately less than $1 \mathrm{~nm}$, crystalline nature, and high lateral area was synthesized. This monolayered $\mathrm{Zn}_{2}(\mathrm{bim})_{4}$ nanosheet colloid, when dropped on porous ceramic $\left(\alpha-\mathrm{Al}_{2} \mathrm{O}_{3}\right)$ substrates at high temperature, resulted in a defect-free membrane with several-nanometer thickness. The resultant membrane displayed excellent $\mathrm{H}_{2}$ gas molecule separation abilities, with permeance varying from 760 to a maximum of $3760 \mathrm{GPU}$ together with the variation of $\mathrm{H}_{2} / \mathrm{CO}_{2}$ selectivity from 53 to a maximum of 291. Later reports from the same group showed $\mathrm{H}_{2}$ permeance of up to $8 \times 10^{-7} \mathrm{~mol} \mathrm{~m}^{-2} \mathrm{~s}^{-1} \mathrm{~Pa}^{-1}$ with a $\mathrm{H}_{2} / \mathrm{CO}_{2}$ separation factor of 166 upon developing another 2D-MOF membrane from $\mathrm{Zn}_{2}(\mathrm{bim})_{3}(\mathrm{OH})$ nanosheets. ${ }^{47}$ Recently, Zhu et al. ${ }^{448}$ reported a novel defect-free membrane from the 2D-MOF $\mathrm{Ni}_{3}(\mathrm{HITP})_{2}$ (HITP $=2,3,6,7,10,11$-hexaaminotriphenylene) via a filtration-based fabrication method on ordered macroporous anodized aluminum oxide (AAO) (Fig. 36). The corresponding membranes exhibited high mechanical stability, long-lasting characteristics, and good reproducibility, making them potential candidates for practical applications such as enhanced gas separation. Furthermore, gas-separation measurements revealed that the $\mathrm{Ni}_{3}(\mathrm{HITP})_{2}$ membrane could obtain high $\mathrm{CO}_{2}$ gas permeance together with enhanced selectivity over other light gases, particularly $\mathrm{N}_{2}$. The $\mathrm{CO}_{2}$ permeance of the $\mathrm{Ni}_{3}(\mathrm{HITP})_{2}$ membrane was $3.15 \times 10^{-6} \mathrm{~mol} \mathrm{~m}^{-2} \mathrm{~s}^{-1} \mathrm{~Pa}^{-1}$ with pure gas separation factors of 13.6 and 7.8 for $\mathrm{CO}_{2} / \mathrm{N}_{2}$ and $\mathrm{H}_{2} / \mathrm{N}_{2}$ gas pairs, respectively. Apart from pure 2D-MOF-nanosheet-containing membranes, the utilization of 2D-MOFs in mixed-matrix membranes (MMMs) was also reported via the simple blending of 2D-MOFs into a polymer matrix for enhanced gas-separation applications. ${ }^{449-452}$

5.3.4.4. Other applications. Apart from the above-mentioned wide-ranging applications of 2D-MOFs, their effectiveness has been broadened even further because of ever-increasing interest in nanomaterials due to their distinctive physical and chemical properties. The practical applicability of 2D-MOFs has been 

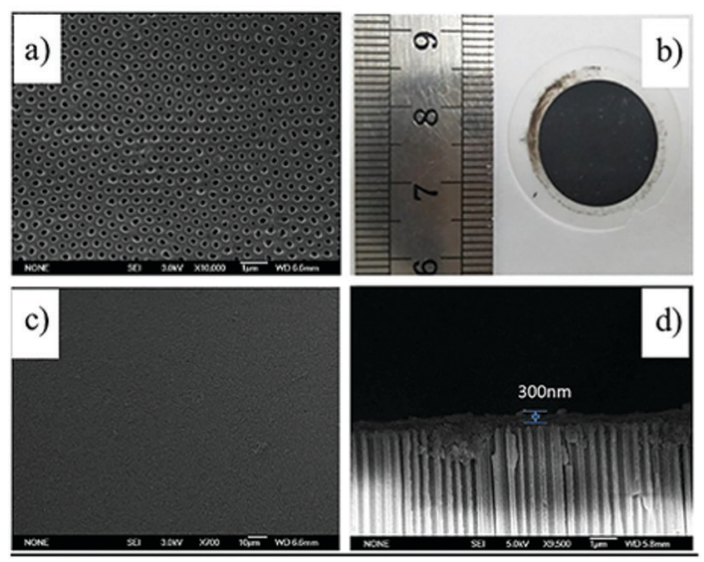

b) e)

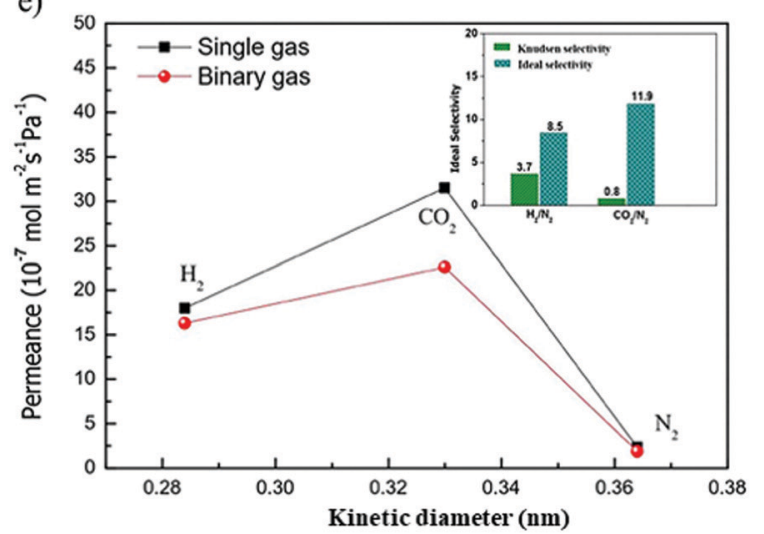

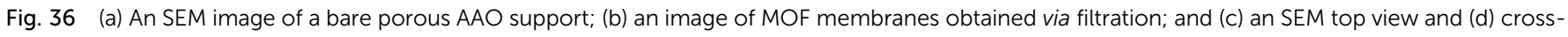

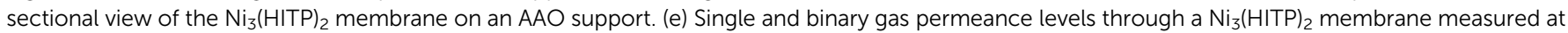

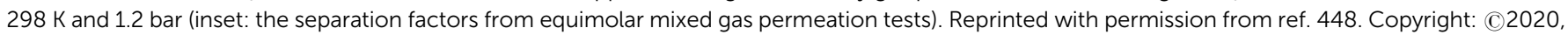
Wiley-VCH Verlag GmbH \& Co. KGaA, Weinheim.

demonstrated in many other fields, such as matrix-assisted laser desorption ionization (MALDI), ${ }^{453}$ gas adsorption, ${ }^{454-456}$ and chiral separation. ${ }^{457}$ For example, Zhi-Yuan $\mathrm{Gu}$ and co-workers developed a $2 \mathrm{D}$-MOF from $\mathrm{Zn}_{2}$ (bim) $)_{4}$ nanosheets, which is stable in water and overcame known matrix difficulties by means of exhibiting an exceptional surface area, efficient photon absorption, and high ionization efficiency. ${ }^{453}$ The newly synthesized 2D MOF $\mathrm{Zn}_{2}(\mathrm{bim})_{4}$ nanosheets showed a layered stable structure, a large accessible surface area, a strong Tyndall effect, and homogeneity as a matrix suspension. Results proved that 2D MOF $\mathrm{Zn}_{2}$ (bim) $)_{4}$ nanosheets can be applied in the analysis of amino acids and hormones as a MALDI-TOF MS matrix. Tang et al. ${ }^{457}$ developed chiral 2D-MOFs for chiral-related separation applications, which are unprecedented in the 2D-MOF family. They used an inverse microemulsion method to prepare chiral L(D)-Thr-Zn nanosheets via reacting chiral $N$-(4-pyridylmethyl)-L(D)-threonine ligands with $\mathrm{Zn}\left(\mathrm{CH}_{3} \mathrm{COO}\right)_{2}$. Due to the presence of a high number of exposed chiral sites on the surface of the newly developed ultrathin chiral L(D)-Thr-Zn nanosheets, extraordinary enantioselectivity during the chiral separation of the racemic $\mathrm{Cr}(\text { oxalate })_{3}{ }^{3-}$ complex and atomically precise $\left[\mathrm{Ag}_{28} \mathrm{Cu}_{12}(\mathrm{SR})_{24}\right]^{4-}(\mathrm{SR}=2,4$-dichlorobenzenethiol) nanoclusters are achieved.

\subsection{Metal-based nanostructured materials}

Metal-based nanomaterials are continuously being investigated for a wide range of applications due to their promising features. ${ }^{458,459}$ The design of nanoscale catalysts has emerged as a significant research area due to their exceptionally high catalytic activities allowing reactions to be achieved more effectively and efficiently. Materials at the nanoscale level provide rich surface textural features, enormous numbers of binding sites, large surface areas, and extremely small sizes, favoring the thermodynamics and kinetics of transportation for heterogeneous reactions. ${ }^{460}$ Metalbased nanostructured materials are also being explored for the production of next-generation artificial enzymes. ${ }^{461}$ More focus has been directed toward designing specific nano-architectures to achieve better performance from these metal-based materials.
A range of methods is available for the synthesis of metal-based nanostructured materials. Metal-based nanostructured materials can be synthesized via hydrothermal, solvothermal, sol-gel, electroless, electrochemical, and physical methods. ${ }^{66}$

As discussed, catalysis is one of the main uses of metalbased nanostructured materials. A continuous increase in the demand for energy, the rapid depletion of conventional energy reservoirs, and rising concerns over the emission of $\mathrm{CO}_{2}$ have increased the challenges and urgency in the energy field. ${ }^{460}$ Metal-based nanostructured materials are extensively being explored to produce alternative clean and renewable energy sources. A range of metal-based nanomaterials has been evaluated and is under consideration for developing robust electrodes that can be effectively applied to water splitting, batteries, and solar cells.

High energy demands have led to more pressure to improve the performances of existing highly demanded lithium-ion batteries. Researchers have focused on improving their lifetimes, sizes, and safety. ${ }^{462}$ Nanostructured metal-oxide-based materials are promising electrode materials for use in high-performance chargestorage devices. A metal-based nanostructured electrode is evaluated as both the anode and cathode to overcome the challenges of conventional electrodes. ${ }^{463}$ In a conventional $\mathrm{LIB}, \mathrm{LiCoO}_{2}$ was used as the cathode material. Controlled morphology plays a crucial role in determining the performance of a material. Powder composed of spherical particles of $\mathrm{LiNi}_{0.8} \mathrm{Co}_{0.2} \mathrm{O}_{2}$ showed a higher tap density compared to irregular particles and the material substantially improved the power density of secondary lithium batteries. ${ }^{464}$ Hierarchical nanostructures of metal-based oxides (such as 3D hierarchical $\mathrm{ZnCo}_{2} \mathrm{O}_{4}$ nanostructures) have emerged as a new trend for the development of high-capacity electrodes for lithium-ion batteries. ${ }^{465}$ Since their commercialization by Sony in the early 1990s, LIBs have achieved tremendous success in bringing portable electronic devices to the market. However, their sustainable development on the grid-scale is hampered due to limited Li resources in nature, and this is causing a continuous increase in cost. ${ }^{466}$ Sodium-ion batteries are in the spotlight to replace 
powerful lithium-ion batteries due to the widespread availability of sodium and its lower cost compared with lithium. ${ }^{467}$ It is essential to note that, in terms of energy densities for SIBs, it is difficult to bypass LIBs because of the low standard electrochemical potential and higher weight of Na. SIBs could be proved to be ideal for those applications where cost is a critical factor compared to energy density. ${ }^{466}$

SIBs also operate similarly to LIBs, based on an intercalation mechanism. SIBs also consist of cathode and anode electrodes separated through an electrolyte. During the charging process, sodium ions are extracted from the cathode and inserted into the anode via the electrolyte. In the discharging process, the electrons leave the anode through an external circuit to reach the cathode, providing electricity to the load, whereas $\mathrm{Na}^{+}$ moves to the cathode during this process. The radius of $\mathrm{Na}^{+}$ $(1.02 \AA)$ is greater than that of $\mathrm{Li}^{+}(0.76 \AA)$, making it challenging to intercalate into electrode materials. ${ }^{468}$ Thus, appropriate electrode materials are required in which fast Na-ion insertion and extraction is possible. However, SIBs are suffering from a lack of appropriate electrode materials. It is important to develop electrode materials that have enough interstitial space within their crystallographic structures and better electrochemical performance. Among the various proposed electrode materials, $\mathrm{Na}_{x} \mathrm{MO}_{2}$ layered transition-metal oxides $(\mathrm{M}=\mathrm{V}, \mathrm{Fe}, \mathrm{Cu}, \mathrm{Co}, \mathrm{Ni}$, $\mathrm{Cr}, \mathrm{Mn}$, and their combinations) are considered to be promising electrode materials for SIBs. Layered metal oxides are considered to be promising electrode materials due to their facile scalable synthesis, simple structures, appropriate operating potentials, and high capacities. ${ }^{469,470}$ Large volume expansion and poor kinetics during the charge-discharge process can severely affect the cyclability and performance of SIBs. One of the effective strategies to deal with the mechanical stress triggered by large volume changes is the design of hollow or porous structures. In response, three-dimensional network-based $\mathrm{Sb}_{2} \mathrm{O}_{3} @ S b$ composite anode materials can help to relieve the volume-change-related stress through their uniform porous networks and provide better transportation channels for $\mathrm{Na}^{+} \cdot{ }^{471}$

The large volume expansion of electrodes can also be buffered via designing 2D metal-oxide materials with large interlayer spacing. The ultrathin nanosheets provide high reversible capacity with enhanced cycling stability and contribute to providing reaction sites for electrons/ions, decreasing the diffusion distance, providing effective diffusion channels, and facilitating fast charge/discharge for sodium and lithium. 2D SnO nanosheet anodes were evaluated for SIBs. The capacity and cyclic stability improved, as the number of atomic SnO layers is decreased in the sheets. ${ }^{472} \mathrm{Sb}$ is a promising anode material, but during the sodiation/desodiation processes, huge volume expansion of $390 \%$ is observed, which hinders its practical use. Nanostructured $\mathrm{Sb}$ in the form of nanorod arrays with large interval spacing displays the great capacity to accommodate volume changes during cycling. ${ }^{473}$ A comparison of various nanostructured metal-based electrodes for various charge storage purposes is shown in Table 3. Overall, well-structured metal or metal-based oxide nanomaterials have the capacity to resolve current issues relating to charge storage devices.
Recently, an immense focus of research has been to produce $\mathrm{H}_{2}$ fuel via water-splitting to replace conventional fossil fuels. This will help to eliminate emissions from the use of carbonaceous species. ${ }^{484}$ Electrochemical method are considered simple water splitting approaches, as these methods only require an applied voltage and water as inputs to produce hydrogen fuel. ${ }^{485}$ The coupling of solar irradiation to electrochemical water splitting has enhanced the performance and reduced the process cost. Due to these reasons, this has become a hot area of research. ${ }^{486}$ During water electrolysis, $\mathrm{H}_{2}$ is produced through the hydrogen evolution reaction at the cathode and $\mathrm{O}_{2}$ is produced through the oxygen evolution reaction at the anode. However, water splitting is not so straightforward, and it requires an efficient catalyst that can facilitate the splitting of water. Metal- and metal-oxide-based catalysts are extensively being explored for water splitting. For the HER reaction, Pt-based catalysts are found to be suitable, whereas for OER reactions, Ir-/Ru-based compounds are found to be benchmark catalysts. Scarcity and high cost have limited the widespread use of these metals. The barrier of noble-metal cost can be mitigated through developing noble-metal nanostructured surfaces that produce more active sites or via depositing monolayers of noble metals on low-cost materials. The alloying of noble metals with other metals has enhanced site-specific activity. ${ }^{484}$ At present, more focus is being placed on developing noble-metalfree catalysts for water splitting. ${ }^{485}$ Usually, an efficient electrocatalyst is characterized by: ${ }^{487}$ a low overpotential; high stability; low production costs; and high electrocatalytic activity.

The nano-structuring of catalysts is an effective tool to boost their surface areas. The electrolysis of water occurs at the surface of a catalyst, and nanostructured catalysts provide more active sites and the better diffusion of ions and electrolytes. ${ }^{484}$ Non-noble metals that are under observation for the development of HER electrocatalysts include nickel (Ni), tungsten (W), iron (Fe), molybdenum (Mo), cobalt (Co), and copper $(\mathrm{Cu}){ }^{487}$ For instance, a noble metal-free catalyst, carbon-decorated $\mathrm{Co}_{3} \mathrm{O}_{4}$ nanoarrays on carbon paper, required a small overpotential of $370 \mathrm{mV}$ to reach a current density of $10 \mathrm{~mA} \mathrm{~cm} \mathrm{~cm}^{-2}$. It can maintain a current density of $100 \mathrm{~mA} \mathrm{~cm}^{-2}$ for $413.8 \mathrm{~h}$ and $86.8 \mathrm{~h}$ under alkaline and acidic conditions, respectively. ${ }^{488}$

Metal-based semiconductor materials play a crucial role in a range of applications. For photoelectrochemical water splitting, the semiconductor material plays a central role in the solar-tohydrogen conversion efficiency. Some critical features are prerequisites when it comes to selecting the right semiconductor material for the photoelectrochemical splitting of water: ${ }^{489}$ an extraordinary capacity to absorb visible light; an appropriate bandgap; suitable valence and conduction band positions; commercial feasibility; and chemical stability.

For an ideal semiconductor for water splitting, the valence band and conduction band edge positions must straddle the oxidation and the reduction potentials of water. Metal oxides have received significant attention among semiconductors due to their wide band gap distributions, remarkable photo-electrochemical stabilities, and favorable band edge positions. ${ }^{490}$ Semiconductor-based photoelectrodes become excited upon light irradiation, and electrons from the valence band move 
Table 3 A comparison of various nanostructured metal- and metal-oxide-based electrode materials for use in charge storage devices

\begin{tabular}{|c|c|c|c|c|c|}
\hline $\begin{array}{l}\text { Nanostructured metal } \\
\text { oxide }\end{array}$ & Synthesis route & $\begin{array}{l}\text { Charge } \\
\text { storage } \\
\text { device }\end{array}$ & Rate capabilities & Retention capacity and cyclability & Ref. \\
\hline $\begin{array}{l}\text { Mesoporous } \mathrm{NiCo}_{2} \mathrm{O}_{4} \\
\text { nanowire arrays }\end{array}$ & $\begin{array}{l}\text { A surfactant-assisted } \\
\text { hydrothermal method and } \\
\text { short post-annealing } \\
\text { treatment }\end{array}$ & LIB, SC & $\begin{array}{l}\approx 1012 \mathrm{~mA} \mathrm{~h} \mathrm{~g}^{-1} \text { at } 0.5 \mathrm{~A} \mathrm{~g}^{-1} \\
1010 \mathrm{~F} \mathrm{~g}^{-1} \text { at } 20 \mathrm{~A} \mathrm{~g}^{-1}\end{array}$ & $\begin{array}{l}\text { Retained } 854 \mathrm{~mA} \mathrm{~h} \mathrm{~g}^{-1} \text { after } \\
100 \text { cycles, negligible specific } \\
\text { capacitance decay after } 5000 \\
\text { cycles at } 8 \mathrm{~A} \mathrm{~g}^{-1}\end{array}$ & 474 \\
\hline $\mathrm{Zn}_{0.25} \mathrm{~V}_{2} \mathrm{O}_{5} \cdot n \mathrm{H}_{2} \mathrm{O}$ nanobelts & $\begin{array}{l}\text { A microwave hydrothermal } \\
\text { method }\end{array}$ & ZIB & $\sim 300 \mathrm{~mA} \mathrm{~h} \mathrm{~g}^{-1}$ at $50 \mathrm{~mA} \mathrm{~g}^{-1}$ & $\begin{array}{l}\text { Capacity retention of } 80 \% \text { at } \\
10 \mathrm{C} \text { after } 1000 \text { cycles }\end{array}$ & 475 \\
\hline $\begin{array}{l}\text { Leaf-like } \mathrm{CuO} \\
\text { nanostructures }\end{array}$ & $\begin{array}{l}\text { In situ precipitation- } \\
\text { induced growth and } \\
\text { thermal annealing }\end{array}$ & LIB & $549 \mathrm{~mA} \mathrm{~h} \mathrm{~g}^{-1}$ at $0.1 \mathrm{~A} \mathrm{~g}^{-1}$ & $95.5 \%$ after 200 cycles & 476 \\
\hline $\begin{array}{l}\text { 3D porous copper skeleton } \\
\text { supported zinc anode }\end{array}$ & Electrodeposition & ZIB & $364 \mathrm{~mA} \mathrm{~h} \mathrm{~g}^{-1}$ at $0.1 \mathrm{~A} \mathrm{~g}^{-1}$ & $\begin{array}{l}\text { Retained a capacity of } 173 \mathrm{~mA} \mathrm{~h} \mathrm{~g}^{-1} \\
\text { at } 0.4 \mathrm{~A} \mathrm{~g}^{-1} \text { after } 300 \text { cycles }\end{array}$ & 477 \\
\hline $\begin{array}{l}\text { ZnO-carbon black nano- } \\
\text { structured anode materials }\end{array}$ & $\begin{array}{l}\text { An atomic layer deposition } \\
\text { method }\end{array}$ & LIB & $2096 \mathrm{~mA} \mathrm{~h} \mathrm{~g}^{-1}$ at $100 \mathrm{~mA} \mathrm{~g}^{-1}$ & $\begin{array}{l}\text { A specific capacity of } 1026 \mathrm{~mA} \mathrm{~h} \mathrm{~g}^{-1} \\
\text { was maintained after } 500 \text { cycles }\end{array}$ & 478 \\
\hline $\mathrm{NiCo}_{2} \mathrm{O}_{4}$ nanoneedle array & $\begin{array}{l}\text { A hydrothermal method } \\
\text { combined with post-heat } \\
\text { treatment }\end{array}$ & SIB & $400 \mathrm{~mA} \mathrm{~h} \mathrm{~g}^{-1}$ at $50 \mathrm{~mA} \mathrm{~g}^{-1}$ & $\sim 215 \mathrm{~mA} \mathrm{~h} \mathrm{~g}^{-1}$ after 50 cycles & 479 \\
\hline $\begin{array}{l}\text { Coral-like nanostructured } \\
\mathrm{Sb}_{2} \mathrm{O}_{3} @ \mathrm{Sb} \text { anode }\end{array}$ & Heating in a furnace & SIB & $\begin{array}{l}497.3 \mathrm{~mA} \mathrm{~h} \mathrm{~g}^{-1} \text { at } 3000 \mathrm{~mA} \mathrm{~g}^{-1} \text {, } \\
724.3 \mathrm{~mA} \mathrm{~h} \mathrm{~g}^{-1} \text { at } 1000 \mathrm{~mA} \mathrm{~g}^{-1}\end{array}$ & $\begin{array}{l}574.8 \mathrm{~mA} \mathrm{~h} \mathrm{~g}^{-1} \text { at } 100 \mathrm{~mA} \mathrm{~g}^{-1} \text { after } \\
150 \text { cycles }\end{array}$ & 471 \\
\hline $3 \mathrm{D} \mathrm{Fe}_{2} \mathrm{GeO}_{4} / \mathrm{N}-\mathrm{CNSs}$ & $\begin{array}{l}\text { A high-temperature } \\
\text { calcination process }\end{array}$ & SIB & $\begin{array}{l}350 \mathrm{~mA} \mathrm{~h} \mathrm{~g}^{-1} \text { at } 0.1 \mathrm{~A} \mathrm{~g}^{-1} \\
180 \mathrm{~mA} \mathrm{~h} \mathrm{~g}^{-1} \text { at } 22.8 \mathrm{~A} \mathrm{~g}^{-1}\end{array}$ & $\begin{array}{l}\sim 86 \% \text { reversible capacity retention } \\
\text { after } 6000 \text { cycles }\end{array}$ & 480 \\
\hline $\mathrm{MnO}_{2}$ nanoflowers & $\begin{array}{l}\text { A hydrothermal method } \\
\text { and thermal treatment }\end{array}$ & SIB & $487.8 \mathrm{~mA} \mathrm{~h} \mathrm{~g}^{-1}$ at $50 \mathrm{~mA} \mathrm{~g}^{-1}$ & 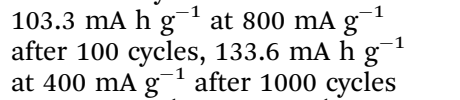 & 481 \\
\hline 2D SnO nanosheet anodes & A hydrothermal method & SIB & $\begin{array}{l}1072 \mathrm{~mA} \mathrm{~h} \mathrm{~g}^{-1} \text { at } 0.1 \mathrm{~A} \mathrm{~g}^{-1} \\
\left(\text { discharge), } 848 \mathrm{~mA} \mathrm{~h} \mathrm{~g}^{-1} \text { at }\right. \\
0.1 \mathrm{~A} \mathrm{~g}^{-1} \text { (charge) }\end{array}$ & $\begin{array}{l}665 \mathrm{~mA} \mathrm{~h} \mathrm{~g}^{-1} \text { at } 0.1 \mathrm{~A} \mathrm{~g}^{-1} \text { after } \\
100 \text { cycles, } 452 \mathrm{~mA} \mathrm{~h} \mathrm{~g}^{-1} \text { at } 1.0 \mathrm{Ag}^{-1} \\
\text { after } 1000 \text { cycles }\end{array}$ & 472 \\
\hline $\mathrm{TiO}_{2} / \mathrm{C}$ nanofibers & Electrospinning & SIB & $164.9 \mathrm{~mA} \mathrm{~h} \mathrm{~g}^{-1}$ at $2000 \mathrm{~mA} \mathrm{~g}^{-1}$ & $\begin{array}{l}\text { Nearly } 100 \% \text { capacity retention } \\
\text { over } 1000 \text { cycles }\end{array}$ & 482 \\
\hline $\mathrm{Sb}$ nanorod arrays & A template method & SIB & $557.7 \mathrm{~mA} \mathrm{~h} \mathrm{~g}^{-1}$ at $20 \mathrm{~A} \mathrm{~g}^{-1}$ & $84 \%$ at $0.2 \mathrm{~A} \mathrm{~g}^{-1}$ over 250 cycles & 473 \\
\hline $\begin{array}{l}\mathrm{CuCo}_{2} \mathrm{O}_{4} \text {-nanodot-inserted } \\
\mathrm{N} \text {-doped carbon nanofibers }\end{array}$ & Electrospinning & SIB & $296 \mathrm{~mA} \mathrm{~h} \mathrm{~g}^{-1}$ at $5000 \mathrm{~mA} \mathrm{~g}^{-1}$ & $\begin{array}{l}314 \mathrm{~mA} \mathrm{~h} \mathrm{~g}^{-1} \text { at } 1000 \mathrm{~mA} \mathrm{~g}^{-1} \\
\text { after } 1000 \text { cycles }\end{array}$ & 483 \\
\hline
\end{tabular}

to the unoccupied conduction band. Some of the generated electrons at the cathode surface reduce protons to hydrogen gas, whereas holes at the photoanode produce oxygen gas via water splitting. ${ }^{490}$ As a result, various nanostructured metal oxides can be used as photoelectrode materials, such as $\mathrm{WO}_{3},{ }^{491} \mathrm{Cu}_{2} \mathrm{O},{ }^{492}$ $\mathrm{TiO}_{2},{ }^{493} \mathrm{ZnO},{ }^{494} \mathrm{SnO}_{2},{ }^{495} \mathrm{BiVO}_{4},{ }^{496}$ and $\alpha-\mathrm{Fe}_{2} \mathrm{O}_{3},{ }^{490}$ for the efficient splitting of water. As discussed, the nano-structuring of semiconductors can significantly impact the electrode photoelectrochemical performance during water splitting.

Metal-based nanomaterials have been used for the development of sensitive sensors. These metal-based sensors can replace the complex and expensive instruments that are conventionally used for the sensing of analytes. Metal-oxide-based sensors have the interesting characteristics of low detection limits, low cost, high sensitivity, and facile operation. ${ }^{497}$ Mostly, semiconducting metal-oxide-based sensors are used for the sensing of toxic, flammable, and exhaust gases. Semiconductor metal oxides with a size in the range of 1-100 $\mathrm{nm}$ have been significantly investigated as gas sensors due to their size-dependent properties. The geometry and size of a nanomaterial can considerably affect the hole and electron movement in semiconductors. ${ }^{498}$ The surfaceto-volume ratio and surface area are substantially enhanced at the nanoscale level, and this is amazingly beneficial for sensing. Chemiresistive semiconducting metal oxides are potential candidates for gas sensing due to the following features: ${ }^{499}$ rapid response times; fast recovery times; low cost; simple electronic interfaces; user-friendliness and low maintenance; and abilities to sense a wide range of gases.

Electrode materials decorated with metal- or metal-oxidebased nanostructured materials have shown better responses and selectivity for determining various analytes over conventional electrode materials. The nano-sized metal structures act as an electrocatalyst and electronic wires to provide rapid electron transfer between the transducers and analyte molecules. ${ }^{500}$ The electrochemical redox reaction of $\mathrm{H}_{2} \mathrm{O}_{2}$ can be improved via the thermally controlled anchoring of Pt NPs on the electrode surface. $^{501}$

Currently, researchers are not just concentrating on the development of randomly shaped nanomaterials; instead, they are very focused on and interested in the rational design of materials with controlled nano-architectures for boosting their performances for specific applications. As a result, extensive research has been carried out to develop metal-based materials with controlled dimensions to achieve better catalytic responses. Particle morphology is a crucial factor in the performance of nanomaterials for specific applications. Laifa Shen et al. rationally designed an electrode architecture via growing mesoporous $\mathrm{NiCo}_{2} \mathrm{O}_{4}$ nanowire arrays on carbon textiles, which boosted the electrode performance (Fig. 37). ${ }^{474}$

The same materials with different morphologies can produce different outcomes. For instance, $\mathrm{MnO}_{2}$ nanoflowers have provided high initial sodium-ion storage capacity compared 


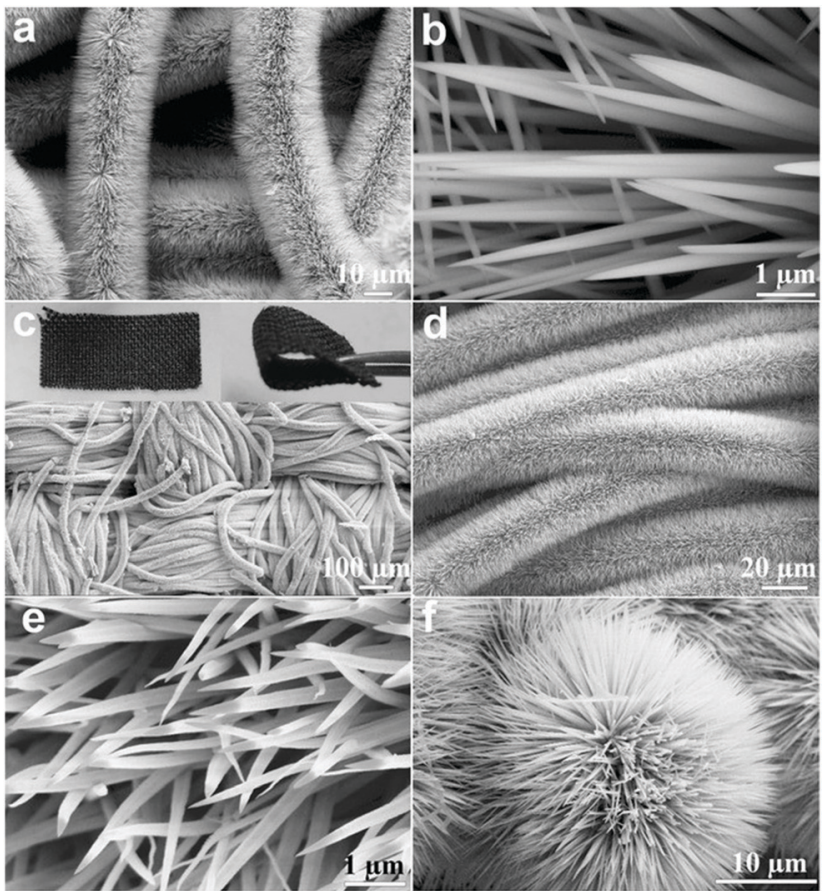

Fig. 37 (a) Low- and (b) high-magnification SEM images of a NiCo-precursor NWA/carbon textile composite, showing the nanowires completely surrounding the carbon microfiber core. (c and d) Low- and (e) high-magnification SEM images of a crystalline $\mathrm{NiCO}_{2} \mathrm{O}_{4} \mathrm{NWA}$ /carbon textile composite. (f) An SEM image of the urchin-like $\mathrm{NiCO}_{2} \mathrm{O}_{4}$ microsphere prepared in the absence of carbon textiles. Reprinted with permission from ref. 474. Copyright: (C2013, WILEY-VCH Verlag GmbH \& Co. KGaA, Weinheim.

with $\mathrm{MnO}_{2}$ nanorods. ${ }^{481}$ Radha Narayanan and Mostafa A. El-Sayed have analyzed various nanoscale morphologies of Pt, such as tetrahedral, cubic, and near-spherical nanoparticles. The highest rate constant is observed with tetrahedral nanoparticles and the lowest rate constant was observed with cubic nanoparticles, whereas spherical nanoparticles exhibited an intermediate rate constant during catalysis. ${ }^{502}$ Xiaowei Xie et al. found that $\mathrm{Co}_{3} \mathrm{O}_{4}$ nanorods show high activity compared to conventional $\mathrm{Co}_{3} \mathrm{O}_{4}$ nanoparticles for the low-temperature oxidation of $\mathrm{CO}^{503}$ The catalytic activity of metal-based nanomaterials is strongly affected by their shape. ${ }^{504}$ Shape-defined mesoporous materials $\left(\mathrm{TiO}_{2}\right)$ have shown superior photoanode activities (Fig. 38). ${ }^{505}$ As a result, in the literature, several nanostructured morphologies of metal-based materials, such as nanotubes, ${ }^{506,507}$ nanorods, ${ }^{508,509}$ nanoflowers, ${ }^{510}$ nanosheets, ${ }^{511}$ nanowires, ${ }^{512}$ nanocubes, ${ }^{513}$ nanospheres, ${ }^{514,515}$ nanocages, ${ }^{516}$ and nanoboxes, ${ }^{517}$ have been reported for a range of applications.

Hollow nanostructures have surfaced as an amazing class of nanostructured material, and they have received significant attention from researchers. Hollow nanostructures have the unique features of: ${ }^{518,519}$ low density; abundant inner void spaces; large surface areas; and the ability to act as nanoscale containers with high loading capacity, nanoreactors, and nanocarriers.

Various metal-based hollow nanostructures, such as hollow $\mathrm{SnO}_{2},{ }^{520}$ hollow palladium nanocrystals, ${ }^{521} \mathrm{Co}-\mathrm{Mn}$ mixed oxide double-shell hollow spheres, ${ }^{521}$ hollow $\mathrm{Cu}_{2} \mathrm{O}$ nanocages, ${ }^{522}$ three-dimensional hollow $\mathrm{SnO}_{2} @ \mathrm{TiO}_{2}$ spheres, ${ }^{523}$ hollow $\mathrm{ZnO} /$ $\mathrm{Co}_{3} \mathrm{O}_{4}$ nano-heterostructure, ${ }^{524}$ triple-shell hollow $\alpha-\mathrm{Fe}_{2} \mathrm{O}_{3},{ }^{525}$ and hierarchical hollow Mn-doped $\mathrm{Ni}(\mathrm{OH})_{2}$ nanostructures, ${ }^{526}$ have been developed for various applications. The presence of nanoscale hollow interiors and functional shells imparts them with great potential for gas sensing, catalysis, biomedicine, energy storage, and conversion. ${ }^{519}$

From this discussion, it can be concluded that metal-based nanostructured materials have great potential compared to their bulk counterparts. The conversion of materials to the nanoscale is not enough to achieve high performance with better selectivity. Now, research is switching from conventional nanomaterials to more advanced and smartly designed nanomaterials. In modern research, nanomaterials are being designed with better-controlled morphologies and regulated features.

\subsection{Core-shell nanoparticles}

Nanoparticles can be categorized as simple, composite, or coreshell examples based on whether the nanoparticle consists of a single material or multiple materials. Nanoparticles consisting of single materials are generally called simple nanoparticles. Core-shell and composite nanoparticles are nanoparticles that consist of two or more materials. More precisely, core-shell is a term used for nanomaterials that comprise an inner material, making a core, and an outer material, forming a shell around the core material. Core-shell nanoparticles of different combinations can be developed, such as organic/organic, inorganic/ organic, inorganic/inorganic, and organic/inorganic combinations. Fig. 39 demonstrates different classes of core-shell nanomaterials. $^{527}$

A spherical nanoparticle core-shell nanostructure is a practical way to introduce multiple functionalities on the nanoscopic length scale. ${ }^{528}$ The properties arising from the core or shell can be different, and these properties can be tuned via controlling the ratio of the constituent materials. The shape, size, and composition play a critical role in tuning the core-shell nanoparticle properties. ${ }^{529}$ The shell material can help to improve the chemical and thermal stabilities of the core material. The core-shell design has become effective where an inexpensive material cannot be used directly due to its instability or easily oxidizable nature. The core can consist of an easily oxidizable inexpensive metal, whereas the shell might consist of noble metals, oxides, polymers, or silica. ${ }^{530}$ For instance, magnetic nanoparticles when prepared can be sensitive toward air, acids, and bases. Magnetic nanoparticles can be protected via coating with organic or inorganic shells. $^{528}$

Core-shell metal nanoparticles are an emerging nanostructured material with great potential in the fields of energy and catalysis. ${ }^{531}$ The first report of core-shell nanoparticles (2007) for supercapacitor applications consisted of a polyaniline/ multi-walled-carbon-nanotube composite (PANI/MWNTs). ${ }^{532}$ Metal-based core-shell structured nanoparticles have shown enhanced catalytic performance due to their shape-controlled properties. ${ }^{533}$ Ming-Yu Kuo et al. developed $\mathrm{Au} @ \mathrm{Cu}_{2} \mathrm{O}$ coreshell particles with controllable shell thicknesses that acted as a dual-functional catalyst. The shell thickness of $\mathrm{Cu}_{2} \mathrm{O}$ 


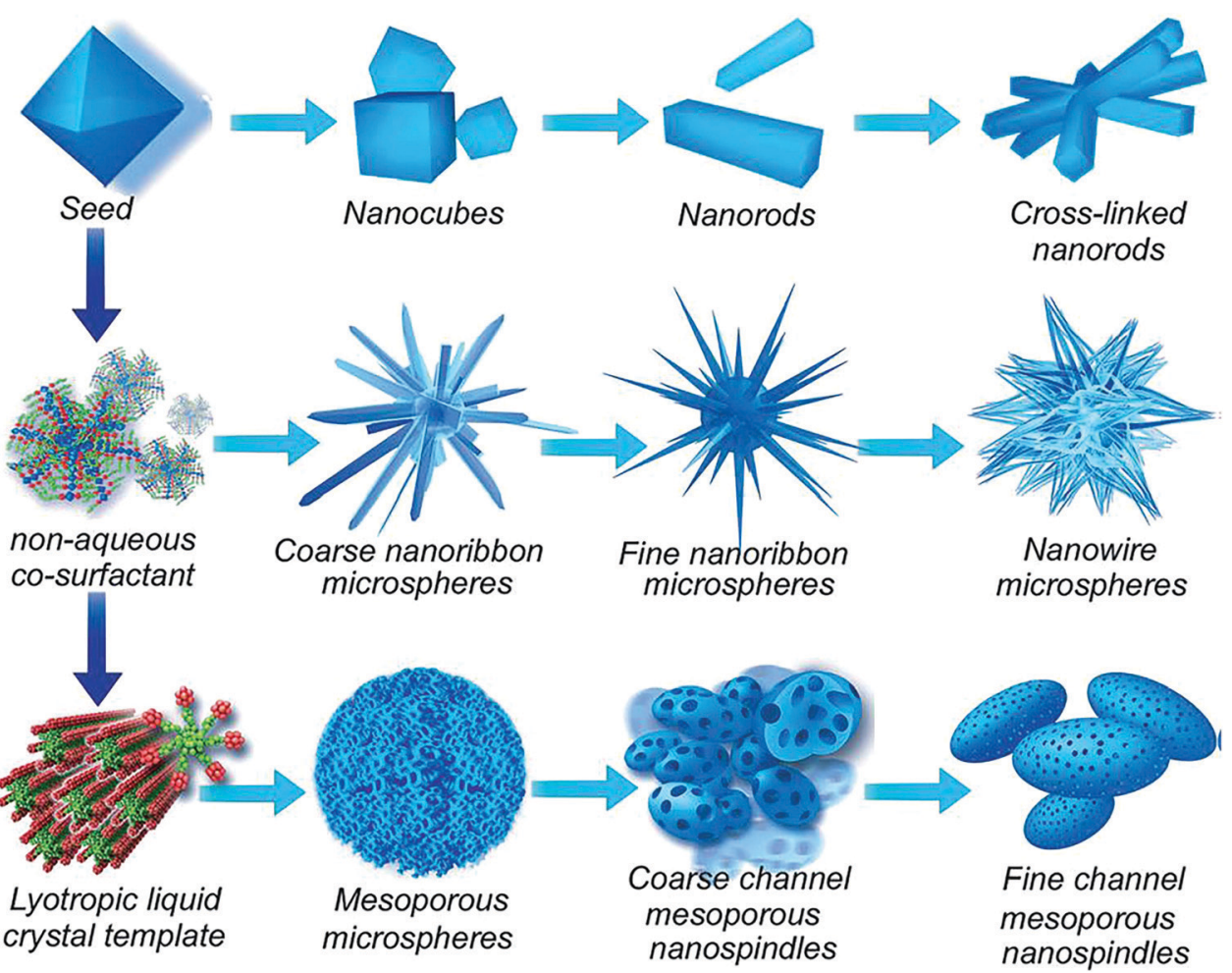

Fig. 38 A schematic illustration of the controlled chemical growth of $\mathrm{TiO}_{2}$ nanostructures with desired morphologies. (top row) Path I: the direct growth of nanocubes, rectangular nanorods, and cross-linked nanorods synthesized from seeds in aqueous solutions; (middle row) path II: 3D dendritic structures synthesized from solution with the addition of a non-aqueous co-surfactant; and (bottom row) path III: shape-defined mesoporous TiO 2 nanostructures synthesized from solutions with lyotropic liquid-crystal templates. Reprinted with permission from ref. 505. Copyright: (C) 2016, WILEY-VCH Verlag GmbH \& Co. KGaA, Weinheim.

increased with an increasing concentration of $\mathrm{Cu}^{2+}$ precursor. The thicknesses of the shells of $\mathrm{Au} @ \mathrm{Cu}_{2} \mathrm{O}-1.5(12.2 \pm 1.7 \mathrm{~nm})$, $\mathrm{Au} @ \mathrm{Cu}_{2} \mathrm{O}-2(13.2 \pm 1.8 \mathrm{~nm}), \mathrm{Au} @ \mathrm{Cu}_{2} \mathrm{O}-3(18.2 \pm 2.2 \mathrm{~nm})$, and $\mathrm{Au} @ \mathrm{Cu}_{2} \mathrm{O}-4(20.8 \pm 2.5 \mathrm{~nm})$ due to various concentrations are shown in Fig. 40. ${ }^{534}$ A NiO@ $\mathrm{SiO}_{2}$ core-shell catalyst provided a higher yield of acrylic acid from acetylene hydroxycarbonylation. ${ }^{535}$ Core-shell architecture can be used to prevent active metal

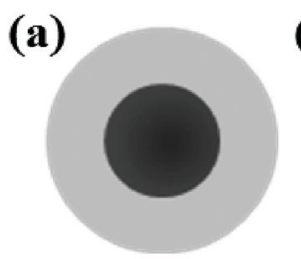

(d)

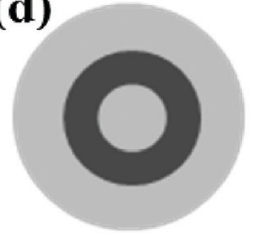

(e)

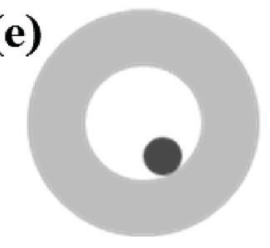

Fig. 39 Different core/shell nanoparticles: (a) spherical core/shell nanoparticles; (b) hexagonal core/shell nanoparticles; (c) multiple small core materials coated with a single shell material; (d) a nanomatryushka material; and (e) a movable core within a hollow shell material. Reprinted with permission from ref. 527. Copyright: (C)2012, American Chemical Society. nanoparticles from oxidation during operation. For instance, a plasmonic photocatalyst was developed that consisted of silver nanoparticles embedded in titanium dioxide. The direct contact of $\mathrm{Ag}$ with $\mathrm{TiO}_{2}$ could lead to its oxidization; this is prevented via developing core-shell architecture in which $\mathrm{Ag}$ is used as the core and $\mathrm{SiO}_{2}$ is used as a shell to protect it. ${ }^{536}$ Another excellent option is to replace an expensive core with a non-noble metal to reduce the core-shell cost while using a thin layer of a noble metal that consumes a small amount of metal as the shell. This will ensure the prolonged stability of the catalyst during operation. ${ }^{533}$ Overall, core-shell morphologies provide better catalytic activity due to the synergistic effect of the metallic core-shell components. ${ }^{152}$

Among the several classes of nanomaterials, core-shell nanoparticles are found to be more promising for different biomedical applications. For instance, magnetic nanoparticles are considered to be useful for biomedical applications due to the following reasons: (a) aggregation is prevented due to superparamagnetism; (b) delivery and separation can be controlled using an external magnetic field; (c) they can be appropriately dispersed; and (d) there is the possibility of functionalization. A range of magnetic nanoparticles is available, such as $\mathrm{NiO}, \mathrm{Ni}$, Co, and $\mathrm{Mn}_{3} \mathrm{O}_{4}$. The most famous example is iron oxide, but uncoated iron oxides are unstable under physiological conditions. This may result in controlled drug delivery failure due to improper ligand surface binding and the promotion of the formation of harmful free radicals. Therefore, the formation of 

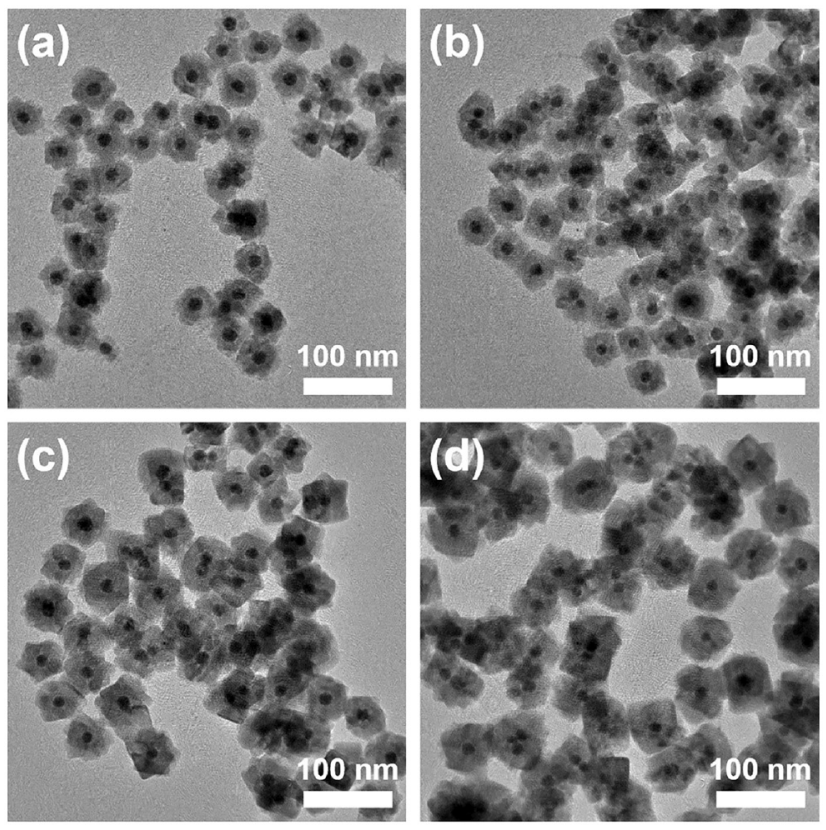

Fig. 40 TEM images of (a) Au@ $\mathrm{Cu}_{2} \mathrm{O}-1.5$, (b) $\mathrm{Au} @ \mathrm{Cu}_{2} \mathrm{O}-2$, (c) $\mathrm{Au}\left(\mathrm{aCu}_{2} \mathrm{O}-\right.$ 3, and (d) $\mathrm{Au}_{\mathrm{aC}} \mathrm{Cu}_{2} \mathrm{O}-4$. Reprinted with permission from ref. 534. Copyright: (C)2018, Elsevier B.V. All rights reserved.

shells around magnetic nanoparticles has tremendous significance for biomedical applications. ${ }^{537}$ One of the approaches is to use gold shells on magnetic nanoparticles. Au NPs are also called surface plasmons and they substantially enhanced the absorption of light in the visible and near-infrared regions. Thus, coating magnetic nanoparticles with a Au shell can result in a core-shell nanostructure that displays both optical and magnetic functionality in combination. ${ }^{529}$

Numerous biocompatible core-shell nanoparticles are being developed for photothermal therapy, as core-shell materials are found to be useful for photothermal therapy. Hui Wang et al. have developed bifunctional core-shell nanoparticles for dualmodal imaging-guided photothermal therapy. The core-shell nanoparticles consist of a magnetic $\sim 9.1 \mathrm{~nm}$ core of $\mathrm{Fe}_{3} \mathrm{O}_{4}$ covered by an approximately $3.4 \mathrm{~nm}$ fluorescent carbon shell. The $\mathrm{Fe}_{3} \mathrm{O}_{4}$ core leads to superparamagnetic behavior, whereas the carbon shell provides near-infrared (NIR) fluorescence properties. The bifunctional nanoparticles have shown dualmodal imaging capacity both in vivo and in vitro. The iron oxide-carbon core-shell nanoparticles absorbed and converted near-infrared light to heat, facilitating photothermal therapy. ${ }^{538}$ Au-Based core-shell structures are also being prepared for photothermal therapy. Bulk gold is biocompatible, but Au NPs can accumulate in the spleen and liver, causing severe toxicity. Koo Chul Kwon et al. have developed Au-NP-based core-shell structures that did not result in any gross or histological lesions in the major organs of mice, which revealed that this is a potent and safe agent for photothermal cancer therapy. The core-shell nanoparticles consisted of proteinticle/gold (PGCS-NP) and were developed via proteinticle surface engineering. PGCS-NP was injected intravenously into mice with tumors, and the injected
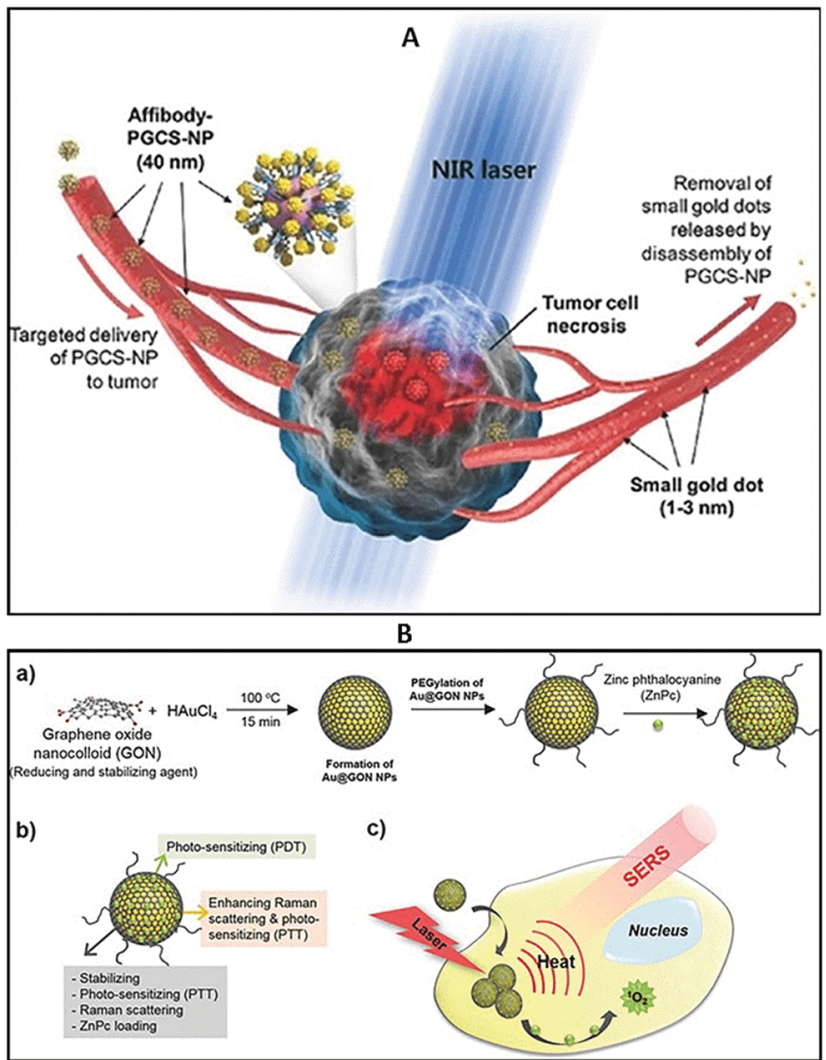

Fig. 41 (A) PGCS-NPs $(40 \mathrm{~nm})$ for the excellent photothermal therapy of cancer. Reprinted with permission from ref. 539. Copyright: (C)2014, WILEY-VCH Verlag GmbH \& Co. KGaA, Weinheim. (B) The synthetic procedure (a), multifunctionality (b), and theranosis process (c) of $\mathrm{ZnPc}-$ PEG-Au@GON NPs. Reprinted with permission from ref. 540. Copyright: (C)2015, WILEY-VCH Verlag GmbH \& Co. KGaA, Weinheim.

core-shell nanoparticles successfully reached the EGFR-expressing tumor cells. The tumor size was significantly reduced upon exposure to near-infrared laser irradiation (Fig. 41). No accumulation of Au NPs was observed in the mice organs, which indicated that PGCS-NP disassembled into many tiny gold dots, which were easily excreted by the kidneys and liver without causing any toxicity. ${ }^{539}$ In another example, multifunctional Au@graphene oxide nanocolloid core@shell nanoparticles were developed, in which the core and shell consisted of gold and a graphene oxide nanocolloid, respectively. The developed core-shell structure showed multifunctional properties, allowing Raman bioimaging and photothermal/photodynamic therapy with low toxicity. ${ }^{540}$ Apart from this, numerous other core-shell nanoparticles, such as polydopamine-mesoporous silica core-shell nanoparticles, ${ }^{541}$ AuPd@PVP core-shell nanoparticles, ${ }^{542} \mathrm{Au} @ \mathrm{Cu}_{2-x} \mathrm{~S}$ core-shell nanoparticles, ${ }^{543}$ bismuth sulfide@mesoporous silica core-shell nanoparticles, ${ }^{544}$ and Ag@S-nitrosothiol core-shell nanoparticles, have been used for photothermal therapy. ${ }^{545}$

Due to their unique features and the combination of properties from the shell and core, these core-shell nanoparticles have received considerable interest in many fields, ranging from materials chemistry to the biomedical field. For electrochemical reactions, the core-shell structure conductivity can be enhanced 
via conducting polymers, carbon materials, and metals. Coreshell nanoparticles as electrode materials showed better performance compared to single components. Most of the core materials are prepared via hydrothermal methods, and shells can be prepared via hydrothermal or electrodeposition methods. ${ }^{546}$ Even though significant progress has been made relating to the synthesis methods of core-shell materials, a major challenge is the high-quality production of core-shell materials in more effective ways for required applications, specifically biomedical applications.

\section{Challenges and future perspectives}

Currently, huge numbers of theoretical and experimental literature studies of nanomaterials and nanotechnology have been witnessed. Future technologies depend upon how effectively materials can be manipulated on the nanoscale for various applications. However, at the same time, the development and effective utilization of nanomaterials involve many challenges. Some of the critical challenges are mentioned below:

(a) The presence of defects in nanomaterials can affect their performance and their inherent characteristics can be compromised. For instance, carbon nanotubes are one of the strongest materials that are known. However, impurities, discontinuous tube lengths, defects, and random orientations can substantially impair the tensile strength of carbon nanotubes. ${ }^{547}$

(b) The synthesis of nanomaterials through cost-effective routes is another major challenge. High-quality nanomaterials are generally produced using sophisticated instrumentation and harsh conditions, limiting their large-scale production. This issue is more critical for the synthesis of 2D nanomaterials. Most of the methods that have been adopted for large-scale production are low cost, and these methods generally produce materials with defects that are of poor quality. The controlled synthesis of nanomaterials is still a challenging job. For example, a crucial challenge associated with carbon nanotube synthesis is to achieve chiral selectivity, conductivity, and precisely controlled diameters. ${ }^{548,549}$ Obtaining structurally pure nanomaterials is the only way to achieve the theoretically calculated characteristics described in the literature. More focused efforts are required to develop new synthesis methods that overcome the challenges associated with conventional methods.

(c) The agglomeration of particles at the nanoscale level is an inherent issue that substantially damages performance in relevant fields. Most nanomaterials start to agglomerate when they encounter each other. The process of agglomeration may be due to physical entanglement, electrostatic interactions, or high surface energy. ${ }^{550}$ CNTs undergo van der Waals interactions and form bundles, making it difficult to align or properly disperse them in polymer matrices. ${ }^{159}$ Similarly, graphene agglomeration is triggered by the basal planes of graphene sheets due to $\pi-\pi$ interactions and van der Waals forces. Due to severe agglomeration, the high surface areas and other unique graphene features are compromised. These challenges hinder the practical application of high-throughput electrode materials or composite materials for various applications. ${ }^{551}$

(d) The efficiency of nanomaterials can be tuned via developing 3D architectures. 3D architectures have been tried with several nanomaterials, such as graphene, to improve their inherent features. 3D architectures of 2D graphene have provided high specific surface areas and fast mass and electron transport kinetics. This has become possible due to the combination of the exceptional intrinsic properties of graphene and 3D porous structures. ${ }^{194,552}$ The combination of graphene and CNT assemblies into 3-D architectures has emerged as the most investigated nanotechnology research area. Porous architectures of other nanomaterials can be developed to enhance their catalysis performance through providing nanomaterial interior availability.

(e) 2D ultrathin materials are an outstanding class of nanomaterial with promising theoretical properties; however, very little experimental evaluation of these materials has been done, apart from the case of graphene. The synthesis and stability of 2D ultrathin materials are some of the major challenges associated with them. In the future, more focus is anticipated to be placed on their synthesis and practical utilization.

(f) Nanomaterial utilization in industry is being increased, and there is also demand for nanoscale material production at higher rates. Moreover, nanotechnology research has vast horizons; the exploration of new nanomaterials with fascinating features will continue and, in the future, more areas will be discovered. One of the significant concerns relating to nanomaterials that cannot be overlooked is their toxicity, which is still poorly understood, and this is a serious concern relating to their environmental, domestic, and industrial use. The extent to which nanoparticle-based materials can contribute to cellular toxicity is unclear. ${ }^{553}$ There is a need for the scientific community to put efforts into reducing the knowledge gap between the rapid development of nanomaterials and their possible in vivo toxicity. A proper and systematic understanding of the interaction of nanomaterials with cells, tissues, and proteins is critical for the safe design and commercialization of nanotechnology. ${ }^{14}$

The future of advanced technology is linked with advancements in the field of nanotechnology. The dream of clean energy production is becoming possible with the advancement of nanomaterialbased engineering strategies. These materials have shown promising results, leading to new generations of hydrogen fuel cells and solar cells, acting as efficient catalysts for water splitting, and showing excellent capacity for hydrogen storage. Nanomaterials have a great future in the field of nanomedicine. Nanocarriers can be used for the delivery of therapeutic molecules.

\section{Conclusions}

Nanomaterials have a long history, and people have utilized them unknowingly. In academics, the concept of modern nanotechnology was introduced by Feynman's famous talk entitled “There's Plenty of Room at the Bottom". After this, much progress in nanotechnology has been witnessed, and the area is continuously expanding into various fields. Materials 
are generally considered nanomaterials when they have any dimension in the range of 1-100 $\mathrm{nm}$. Two main approaches are used for the synthesis of nanomaterials. One of the main approaches involves top-down approaches, which consist of various methods, including mechanical milling, electrospinning, lithography, sputtering, arc discharge, and laser ablation methods. The second approach involves bottom-up approaches, which include chemical vapor deposition (CVD), solvothermal and hydrothermal, sol-gel, and reverse micelle methods. Nanomaterials have exhibited a range of unique features that are substantially different from their bulk counterparts. Nanomaterials possess high surface areas, magnetism, quantum effects, antimicrobial activity, and high thermal and electrical conductivities. At the nanoscale, metal-based materials have shown substantially high catalytic activities, and the better dispersion of these catalysts can be achieved via dispersion on $2 \mathrm{D}$ sheets of other nanomaterials, enhancing the overall performance of metal-based catalysts. The nanomaterials family includes carbon-based nanomaterials, nanoporous materials, core-shell materials, ultrathin 2-dimensional nanomaterials, and metal-based nanomaterials. Among these, carbon-based nanomaterials are a fascinating class of nanomaterial, consisting of fullerenes, carbon nanotubes, carbon-based quantum dots, graphene, and carbon nanohorns. Moreover, the surfaces of carbon-based nanomaterials can be functionalized further to tune their properties for certain applications. CNTs and graphene are highly acknowledged members of the carbonbased nanomaterials family and they have been extensively explored for various applications due to their high surface areas, rapid charge transfer properties, and high mechanical strength. Carbon quantum dots have received great attention in the fields of sensing, nanomedicine, and bioimaging. After graphene isolation in 2004 from graphite, large interest in ultrathin 2D materials was witnessed due to their numerous unprecedented features. As a result, several ultrathin nanomaterials have been reported, including silicene, borophene, antimonene, MXenes, 2D MOF nanosheets, and boron nitride nanosheets. Apart from the outstanding characteristics of ultrathin 2D materials, the experimental evaluation of these materials is still at an early stage; however, these materials are being rapidly explored for useful applications. Designing catalysts on the nanoscale has become a hot area of research, and many metal-based nanostructured materials are being developed as nano-catalysts. Catalysts on the nanoscale provide huge surface areas, enormous numbers of binding sites, and rich surface textural features. All these features, along with the small size, favor the thermodynamics and kinetics of transportation during heterogeneous reactions. Layered metal-oxide-based materials are being explored as electrode materials in energy conversion and storage devices. Semiconductor metal oxide materials are being tuned on the nanoscale to enhance their performance for catalyzing water to produce clean energy. More focus is currently being placed on producing nanomaterials with controlled morphologies and nanoscale dimensions to achieve the desired outcomes as a result of well-organized nanostructures. By using nanotechnology, some commercial devices have already been introduced. Much more progress is anticipated, with nanomaterials being introduced into next-generation devices to cope with future high energy demands and playing a more active role in biosensors and nanomedicine to fight against existing and novel diseases. A few examples of synthetic nanoparticles, such as Abraxane and Doxil, are being used for biomedical applications. These nanoparticles are clinically approved therapeutics. Submicron-sized $\mathrm{TiO}_{2}$ particles are a key component of white paints. Zinc oxide and $\mathrm{TiO}_{2}$ oxide nanoparticles are used in commercial sunscreens. ${ }^{554}$ A huge quantity of titania is industrially produced every year. In commercial LCDs, color pigments with crystal sizes of less than $40 \mathrm{~nm}$ are introduced to provide better color purity, brightness, and contrast for high-definition television-based applications. ${ }^{555}$ Apart from a few examples, most nanomaterials are being engineered for labscale applications, and serious efforts are required to bring them to the commercial market. Another major challenge associated with modern nanotechnology is to find alternatives to the use of endangered and resource-limited materials in nanomaterial production. In the coming years, 44 elements out of 118 elements will face supply limitations. Precious metals, phosphorous, and rareearth elements are on the list of critical elements. There is a dire need to reduce the dependency on endangered and critical elements. For instance, in batteries, efforts are ongoing to replace critical lithium ions with other abundant metal ions. Due to the abundant availability of carbon sources, carbon-based nanomaterials are a good choice for large-scale production for various applications. Core-shell based morphologies can also prove to be effective for reducing the utilization of critical elements for various applications. Nanotechnology can play an active role in the decontamination of water and the recycling of wastewater. Future challenges faced by modern society can be fixed with a better understanding and the rapid development of nanotechnology.

\section{Conflicts of interest}

There are no conflicts to declare.

\section{Acknowledgements}

The authors would like to acknowledge support provided by the King Fahd University of Petroleum and Minerals.

\section{References}

1 F. J. Heiligtag and M. Niederberger, Mater. Today, 2013, 16, 262-271.

2 P. Walter, E. Welcomme, P. Hallégot, N. J. Zaluzec, C. Deeb, J. Castaing, P. Veyssière, R. Bréniaux, J.-L. Lévêque and G. Tsoucaris, Nano Lett., 2006, 6, 2215-2219.

3 J. Jeevanandam, A. Barhoum, Y. S. Chan, A. Dufresne and M. K. Danquah, Beilstein J. Nanotechnol., 2018, 9, 1050-1074.

4 I. Freestone, N. Meeks, M. Sax and C. Higgitt, Gold Bull., 2007, 40, 270-277.

5 A. Santamaria, Nanotoxicity, Humana Press, Totowa, NJ, 2012, pp. 1-12.

6 R. P. Feynman, Eng. Sci., 1960, 23, 22-36. 
7 S. Bayda, M. Adeel, T. Tuccinardi, M. Cordani and F. Rizzolio, Molecules, 2019, 25, 112.

8 M. Nasrollahzadeh, S. M. Sajadi, M. Sajjadi and Z. Issaabadi, Interface Science and Technology, Elsevier B.V., 2019, vol. 28, pp. 1-27.

9 N. Taniguchi, Proc. Int. Conf. Prod. Eng. Issue PART II, 1974, 18-23.

10 G. Binnig and H. Rohrer, US Pat., US4343993A, 1982.

11 G. Binnig, C. F. Quate and C. Gerber, Phys. Rev. Lett., 1986, 56, 930-933.

12 H.-J. Butt, B. Cappella and M. Kappl, Surf. Sci. Rep., 2005, 59, 1-152.

13 A. C. Marques, M. Vale, D. Vicente, M. Schreck, E. Tervoort and M. Niederberger, Global Challenges, 2021, 2000116.

14 S. Sharifi, S. Behzadi, S. Laurent, M. Laird Forrest, P. Stroeve and M. Mahmoudi, Chem. Soc. Rev., 2012, 41, 2323-2343.

15 Nat. Nanotechnol., 2019, 14, 193.

16 P. N. Sudha, K. Sangeetha, K. Vijayalakshmi and A. Barhoum, Emerging Applications of Nanoparticles and Architecture Nanostructures, Elsevier, 2018, pp. 341-384.

17 W. G. Kreyling, M. Semmler-Behnke and Q. Chaudhry, Nano Today, 2010, 5, 165-168.

18 B. Bhushan, Springer Handbooks, Springer, 2017, pp. 1-19.

19 F. Trotta and A. Mele, Nanosponges: From Fundamentals to Applications, 2019, pp. 1-24.

20 Nanoanalytics, ed. S. Shtykov, De Gruyter, Berlin, Boston, 2018.

21 C. J. Murphy and N. R. Jana, Adv. Mater., 2002, 14, 80-82.

22 P. Moriarty, Rep. Prog. Phys., 2001, 64, 297-381.

23 B. Chen, J. R. G. Evans, H. C. Greenwell, P. Boulet, P. V. Coveney, A. A. Bowden and A. Whiting, Chem. Soc. Rev., 2008, 37, 568-594.

24 P. Khanna, A. Kaur and D. Goyal, J. Microbiol. Methods, 2019, 163, 105656.

25 S. Zhuang, E. S. Lee, L. Lei, B. B. Nunna, L. Kuang and W. Zhang, Int. J. Energy Res., 2016, 40, 2136-2149.

26 T. Prasad Yadav, R. Manohar Yadav and D. Pratap Singh, Nanosci. Nanotechnol., 2012, 2, 22-48.

27 H. Lyu, B. Gao, F. He, C. Ding, J. Tang and J. C. Crittenden, ACS Sustainable Chem. Eng., 2017, 5, 9568-9585.

28 R. Ostermann, J. Cravillon, C. Weidmann, M. Wiebcke and B. M. Smarsly, Chem. Commun., 2011, 47, 442-444.

29 P. S. Kumar, J. Sundaramurthy, S. Sundarrajan, V. J. Babu, G. Singh, S. I. Allakhverdiev and S. Ramakrishna, Energy Environ. Sci., 2014, 7, 3192-3222.

30 P. Du, L. Song, J. Xiong, N. Li, Z. Xi, L. Wang, D. Jin, S. Guo and Y. Yuan, Electrochim. Acta, 2012, 78, 392-397.

31 A. Pimpin and W. Srituravanich, Eng. J., 2012, 16, 37-56.

32 Z. Szabó, J. Volk, E. Fülöp, A. Deák and I. Bársony, Photonics Nanostruct., 2013, 11, 1-7.

33 C.-W. Kuo, J.-Y. Shiu, Y.-H. Cho and P. Chen, Adv. Mater., 2003, 15, 1065-1068.

34 Y. Yin, B. Gates and Y. Xia, Adv. Mater., 2000, 12, 1426-1430. 35 K. Xu and J. Chen, Appl. Nanosci., 2020, 10, 1013-1022.

36 R. Matsumoto, S. Adachi, E. H. S. Sadki, S. Yamamoto, H. Tanaka, H. Takeya and Y. Takano, ACS Appl. Electron. Mater., 2020, 2, 677-682.
37 V. Garg, R. G. Mote and J. Fu, Appl. Surf. Sci., 2020, 526, 146644. 38 P. Ayyub, R. Chandra, P. Taneja, A. K. Sharma and R. Pinto, Appl. Phys. A: Mater. Sci. Process., 2001, 73, 67-73.

39 H. H. Son, G. H. Seo, U. Jeong, D. Y. Shin and S. J. Kim, Int. J. Heat Mass Transfer, 2017, 113, 115-128.

40 H. Wender, P. Migowski, A. F. Feil, S. R. Teixeira and J. Dupont, Coord. Chem. Rev., 2013, 257, 2468-2483.

41 J. Muñoz-García, L. Vázquez, R. Cuerno, J. A. Sánchez-García, M. Castro and R. Gago, Toward Functional Nanomaterials, Springer US, New York, NY, 2009, pp. 323-398.

42 J. H. Nam, M. J. Jang, H. Y. Jang, W. Park, X. Wang, S. M. Choi and B. Cho, J. Energy Chem., 2020, 47, 107-111.

43 M. Nie, K. Sun and D. D. Meng, J. Appl. Phys., 2009, 106, 054314.

44 D. Zhang, K. Ye, Y. Yao, F. Liang, T. Qu, W. Ma, B. Yang, Y. Dai and T. Watanabe, Carbon, 2019, 142, 278-284.

45 C. M. Lieber and C.-C. Chen, Solid State Physics - Advances in Research and Applications, Academic Press, 1994, vol. 48, pp. 109-148.

46 J. M. Jones, R. P. Malcolm, K. M. Thomas and S. H. Botrell, Carbon, 1996, 34, 231-237.

47 F. Liang, M. Tanaka, S. Choi and T. Watanabe, Carbon, 2017, 117, 100-111.

48 F. Liang, T. Shimizu, M. Tanaka, S. Choi and T. Watanabe, Diamond Relat. Mater., 2012, 30, 70-76.

49 N. Li, Z. Wang, K. Zhao, Z. Shi, Z. Gu and S. Xu, Carbon, 2010, 48, 1580-1585.

50 Z.-S. Wu, W. Ren, L. Gao, J. Zhao, Z. Chen, B. Liu, D. Tang, B. Yu, C. Jiang and H.-M. Cheng, ACS Nano, 2009, 3, 411-417.

51 V. Amendola and M. Meneghetti, Phys. Chem. Chem. Phys., 2009, 11, 3805.

52 J. Zhang, M. Chaker and D. Ma, J. Colloid Interface Sci., 2017, 489, 138-149.

53 R. A. Ismail, M. H. Mohsin, A. K. Ali, K. I. Hassoon and S. Erten-Ela, Phys. E, 2020, 119, 113997.

54 J. Chrzanowska, J. Hoffman, A. Małolepszy, M. Mazurkiewicz, T. A. Kowalewski, Z. Szymanski and L. Stobinski, Phys. Status Solidi, 2015, 252, 1860-1867.

55 J. Duque, B. Madrigal, H. Riascos and Y. Avila, Colloids Interfaces, 2019, 3, 25.

56 S. S. Su and I. Chang, Commercialization of NanotechnologiesA Case Study Approach, Springer International Publishing, Cham, 2018, pp. 15-29.

57 H. Park, D. A. Reddy, Y. Kim, S. Lee, R. Ma and T. K. Kim, Chem. - A Eur. J., 2017, 23, 13112-13119.

58 A. C. Jones and M. L. Hitchman, in Chemical Vapour Deposition, ed. A. C. Jones and M. L. Hitchman, Royal Society of Chemistry, Cambridge, 2008, pp. 1-36.

59 K. A. Shah and B. A. Tali, Mater. Sci. Semicond. Process., 2016, 41, 67-82.

60 H. Ago, Frontiers of Graphene and Carbon Nanotubes, Springer, Japan, Tokyo, 2015, pp. 3-20.

61 P. Machac, S. Cichon, L. Lapcak and L. Fekete, Graphene Technol., 2020, 5, 9-17.

62 Q. Wu, W. Wongwiriyapan, J.-H. Park, S. Park, S. J. Jung, T. Jeong, S. Lee, Y. H. Lee and Y. J. Song, Curr. Appl. Phys., 2016, 16, 1175-1191. 
63 X. Wu, G. Q. (Max) Lu and L. Wang, Energy Environ. Sci., 2011, 4, 3565.

64 S. Cao, C. Zhao, T. Han and L. Peng, Mater. Lett., 2016, 169, 17-20.

$65 \mathrm{~J} . \mathrm{Li}, \mathrm{Q}$. Wu and J. Wu, Handbook of Nanoparticles, Springer International Publishing, Cham, 2015, pp. 1-28.

66 A. Chen and P. Holt-Hindle, Chem. Rev., 2010, 110, 3767-3804.

67 L.-Y. Meng, B. Wang, M.-G. Ma and K.-L. Lin, Mater. Today Chem., 2016, 1-2, 63-83.

68 Y. Dong, X. Du, P. Liang and X. Man, Inorg. Chem. Commun., 2020, 115, 107883.

69 Y. Jiang, Z. Peng, S. Zhang, F. Li, Z. Liu, J. Zhang, Y. Liu and K. Wang, Ceram. Int., 2018, 44, 6115-6126.

70 B. Chai, M. Xu, J. Yan and Z. Ren, Appl. Surf. Sci., 2018, 430, 523-530.

71 A. E. Danks, S. R. Hall and Z. Schnepp, Mater. Horiz., 2016, 3, 91-112.

72 T. K. Tseng, Y. S. Lin, Y. J. Chen and H. Chu, Int. J. Mol. Sci., 2010, 11, 2336-2361.

73 M. Parashar, V. K. Shukla and R. Singh, J. Mater. Sci.: Mater. Electron., 2020, 31, 3729-3749.

74 L. Znaidi, Mater. Sci. Eng., B, 2010, 174, 18-30.

75 C. de Coelho Escobar and J. H. Z. dos Santos, J. Sep. Sci., 2014, 37, 868-875.

76 Y. Liu, J. Goebl and Y. Yin, Chem. Soc. Rev., 2013, 42, 2610-2653.

77 W. Li and D. Zhao, Chem. Commun., 2013, 49, 943-946.

78 R. R. Poolakkandy and M. M. Menamparambath, Nanoscale Adv., 2020, 2, 5015-5045.

79 C. T. Kresge, M. E. Leonowicz, W. J. Roth, J. C. Vartuli and J. S. Beck, Nature, 1992, 359, 710-712.

80 J. S. Beck, J. C. Vartuli, W. J. Roth, M. E. Leonowicz, C. T. Kresge, K. D. Schmitt, C. T. W. Chu, D. H. Olson, E. W. Sheppard, S. B. McCullen, J. B. Higgins and J. L. Schlenker, J. Am. Chem. Soc., 1992, 114, 10834-10843.

81 J. Liu, T. Yang, D.-W. Wang, G. Q. Lu, D. Zhao and S. Z. Qiao, Nat. Commun., 2013, 4, 2798.

82 R. Lv, C. Cao, H. Zhai, D. Wang, S. Liu and H. Zhu, Solid State Commun., 2004, 130, 241-245.

83 L. Martins, M. A. Alves Rosa, S. H. Pulcinelli and C. V. Santilli, Microporous Mesoporous Mater., 2010, 132, 268-275.

84 T. Tang, T. Zhang, W. Li, X. Huang, X. Wang, H. Qiu and Y. Hou, Nanoscale, 2019, 11, 7440-7446.

85 B. Szczęśniak, J. Choma and M. Jaroniec, Chem. Commun., 2020, 56, 7836-7848.

86 Y. Yamauchi and K. Kuroda, Chem. - Asian J., 2008, 3, 664-676.

87 S. J. Hurst, E. K. Payne, L. Qin and C. A. Mirkin, Angew. Chem., Int. Ed., 2006, 45, 2672-2692.

88 M. A. Malik, M. Y. Wani and M. A. Hashim, Arabian J. Chem., 2012, 5, 397-417.

89 M. Soleimani Zohr Shiri, W. Henderson and M. R. Mucalo, Materials, 2019, 12, 1896.

90 T.-D. Nguyen, Nanoscale, 2013, 5, 9455.

91 S. Yi, F. Dai, C. Zhao and Y. Si, Sci. Rep., 2017, 7, 9806.
92 R. Jose Varghese, E. H. M. Sakho, S. Parani, S. Thomas, O. S. Oluwafemi and J. Wu, Nanomaterials for Solar Cell Applications, Elsevier, 2019, pp. 75-95.

93 E. Roduner, Chem. Soc. Rev., 2006, 35, 583.

94 A. J. Mannix, X.-F. Zhou, B. Kiraly, J. D. Wood, D. Alducin, B. D. Myers, X. Liu, B. L. Fisher, U. Santiago, J. R. Guest, M. J. Yacaman, A. Ponce, A. R. Oganov, M. C. Hersam and N. P. Guisinger, Science, 2015, 350, 1513-1516.

95 Nanobiotechnology: Concepts and Applications in Health, Agriculture, and Environment, ed. R. S. Tomar, A. Jyoti and S. Kaushik, 1st edn, 2020.

96 A. P. Alivisatos, Science, 1996, 271, 933-937.

97 R. Tomar, A. A. Abdala, R. G. Chaudhary and N. B. Singh, Mater. Today: Proc., 2020, 29, 967-973, DOI: 10.1016/ j.matpr.2020.04.144.

98 L. D. Geoffrion and G. Guisbiers, J. Phys. Chem. Solids, 2020, 140, 109320.

99 S. K. Krishnan, E. Singh, P. Singh, M. Meyyappan and H. S. Nalwa, RSC Adv., 2019, 9, 8778-8881.

100 Q. Wu, W. Miao, Y. Zhang, H. Gao and D. Hui, Nanotechnol. Rev., 2020, 9, 259-273.

101 W. Zhu, Y. Guo, B. Ma, X. Yang, Y. Li, P. Li, Y. Zhou and M. Shuai, Int. J. Hydrogen Energy, 2020, 45, 8385-8395.

102 X. Liu, M. Xu, L. Wan, H. Zhu, K. Yao, R. Linguerri, G. Chambaud, Y. Han and C. Meng, ACS Catal., 2020, 10, 3084-3093.

103 P. Makvandi, C. Wang, E. N. Zare, A. Borzacchiello, L. Niu and F. R. Tay, Adv. Funct. Mater., 2020, 30, 1910021.

104 E. Castro, A. H. Garcia, G. Zavala and L. Echegoyen, J. Mater. Chem. B, 2017, 5, 6523-6535.

105 M. S. Mauter and M. Elimelech, Environ. Sci. Technol., 2008, 42, 5843-5859.

106 L. Chen, S. Zhao, Q. Hasi, X. Luo, C. Zhang, H. Li and A. Li, Global Challenges, 2020, 4, 1900098.

107 R. G. Mendes, A. Bachmatiuk, B. Büchner, G. Cuniberti and M. H. Rümmeli, J. Mater. Chem. B, 2013, 1, 401-428.

108 M. T. Beck and G. Mándi, Fullerene Sci. Technol., 1997, 5, 291-310.

109 X. Fan, N. Soin, H. Li, H. Li, X. Xia and J. Geng, Energy Environ. Mater., 2020, 3, 469-491, DOI: 10.1002/ eem2.12071.

110 S. Goodarzi, T. Da Ros, J. Conde, F. Sefat and M. Mozafari, Mater. Today, 2017, 20, 460-480.

111 P. Ehrenfreund and B. H. Foing, Science, 2010, 329, 1159-1160.

112 J. C. Withers, R. O. Loutfy and T. P. Lowe, Fullerene Sci. Technol., 1997, 5, 1-31.

113 A. Rodríguez-Fortea, A. L. Balch and J. M. Poblet, Chem. Soc. Rev., 2011, 40, 3551.

114 N. A. Kaskhedikar and J. Maier, Adv. Mater., 2009, 21, 2664-2680.

115 A. A. Popov, S. Yang and L. Dunsch, Chem. Rev., 2013, 113, 5989-6113.

116 B. Q. Mercado, C. M. Beavers, M. M. Olmstead, M. N. Chaur, K. Walker, B. C. Holloway, L. Echegoyen and A. L. Balch, J. Am. Chem. Soc., 2008, 130, 7854-7855. 
117 S. Yang and C.-R. Wang, Endohedral Fullerenes, World Scientific, 2014.

118 H. Okada, T. Komuro, T. Sakai, Y. Matsuo, Y. Ono, K. Omote, K. Yokoo, K. Kawachi, Y. Kasama, S. Ono, R. Hatakeyama, T. Kaneko and H. Tobita, RSC Adv., 2012, 2, 10624.

119 H. Bai, H. Gao, W. Feng, Y. Zhao and Y. Wu, Nanomaterials, 2019, 9, 630.

120 O. V. Pupysheva, A. A. Farajian and B. I. Yakobson, Nano Lett., 2008, 8, 767-774.

121 M. Gaboardi, N. Sarzi Amadé, M. Aramini, C. Milanese, G. Magnani, S. Sanna, M. Riccò and D. Pontiroli, Carbon, 2017, 120, 77-82.

122 A. Shokuhi Rad and K. Ayub, Comput. Theor. Chem., 2017, 1121, 68-75.

123 D. Guldi and N. Martin, Comprehensive Nanoscience and Technology, Elsevier, 2011, pp. 379-398.

124 L. Sánchez, N. Martín and D. M. Guldi, Angew. Chem., Int. Ed., 2005, 44, 5374-5382.

125 X. Zhang, Y. Ma, S. Fu and A. Zhang, Nanomaterials, 2019, 9, 1647.

126 N. Martin and G. Bodwell, Acc. Chem. Res., 2019, 52, 2757-2759.

127 K. Harano and E. Nakamura, Acc. Chem. Res., 2019, 52, 2090-2100.

128 A. Montellano, T. Da Ros, A. Bianco and M. Prato, Nanoscale, 2011, 3, 4035.

129 E. Alipour, F. Alimohammady, A. Yumashev and A. Maseleno, J. Mol. Model., 2020, 26, 7.

130 M.-S. Lin, R.-T. Chen, N.-Y. Yu, L.-C. Sun, Y. Liu, C.-H. Cui, S.-Y. Xie, R.-B. Huang and L.-S. Zheng, Colloids Surf., B, 2017, 159, 613-619.

131 K. Minami, K. Okamoto, K. Harano, E. Noiri and E. Nakamura, ACS Appl. Mater. Interfaces, 2018, 10, 19347-19354.

132 K. Minami, K. Okamoto, K. Doi, K. Harano, E. Noiri and E. Nakamura, Sci. Rep., 2015, 4, 4916.

133 S. S. Babu, H. Möhwald and T. Nakanishi, Chem. Soc. Rev., 2010, 39, 4021.

134 N. Furuuchi, R. Shrestha, Y. Yamashita, T. Hirao, K. Ariga and L. Shrestha, Sensors, 2019, 19, 267.

135 X. Fan, N. Soin, H. Li, H. Li, X. Xia and J. Geng, Energy Environ. Mater., 2020, 3, 469-491, DOI: 10.1002/eem2.12071.

136 J. Coro, M. Suárez, L. S. R. Silva, K. I. B. Eguiluz and G. R. SalazarBanda, Int. J. Hydrogen Energy, 2016, 41, 17944-17959.

137 N. Yousfi-Steiner, P. Moçotéguy, D. Candusso and D. Hissel, J. Power Sources, 2009, 194, 130-145.

138 A. R. Tuktarov, A. A. Khuzin and U. M. Dzhemilev, Pet. Chem., 2020, 60, 113-133.

139 I. Jeon, A. Shawky, H.-S. Lin, S. Seo, H. Okada, J.-W. Lee, A. Pal, S. Tan, A. Anisimov, E. I. Kauppinen, Y. Yang, S. Manzhos, S. Maruyama and Y. Matsuo, J. Am. Chem. Soc., 2019, 141, 16553-16558.

140 Y. Takabayashi and K. Prassides, Philos. Trans. R. Soc., A, 2016, 374, 20150320.

141 M. A. Rafiee, F. Yavari, J. Rafiee and N. Koratkar, J. Nanopart. Res., 2011, 13, 733-737.
142 Y. Pan, Z. Guo, S. Ran and Z. Fang, J. Appl. Polym. Sci., 2020, 137, 47538.

143 S. Iijima, Nature, 1991, 354, 56-58.

144 S. Iijima and T. Ichihashi, Nature, 1993, 363, 603-605.

145 I. V. Zaporotskova, N. P. Boroznina, Y. N. Parkhomenko and L. V. Kozhitov, Mod. Electron. Mater., 2016, 2, 95-105.

146 X.-Q. Li, P.-X. Hou, C. Liu and H.-M. Cheng, Carbon, 2019, 147, 187-198.

147 C. Shen, A. H. Brozena and Y. Wang, Nanoscale, 2011, 3, 503-518.

148 V. Popov, Mater. Sci. Eng., R, 2004, 43, 61-102.

149 Z. F. Ren, Science, 1998, 282, 1105-1107.

150 A. G. Rinzler, J. Liu, H. Dai, P. Nikolaev, C. B. Huffman, F. J. Rodríguez-Macías, P. J. Boul, A. H. Lu, D. Heymann, D. T. Colbert, R. S. Lee, J. E. Fischer, A. M. Rao, P. C. Eklund and R. E. Smalley, Appl. Phys. A: Mater. Sci. Process., 1998, 67, 29-37.

151 P. Nikolaev, M. J. Bronikowski, R. K. Bradley, F. Rohmund, D. T. Colbert, K. Smith and R. E. Smalley, Chem. Phys. Lett., 1999, 313, 91-97.

152 N. Baig, M. Sajid and T. A. Saleh, TrAC, Trends Anal. Chem., 2019, 111, 47-61.

153 X.-M. Liu, Z. Dong Huang, S. Woon Oh, B. Zhang, P.-C. Ma, M. M. F. Yuen and J.-K. Kim, Compos. Sci. Technol., 2012, 72, 121-144.

154 T. W. Odom, J.-L. Huang, P. Kim and C. M. Lieber, Nature, 1998, 391, 62-64.

155 K. Balasubramanian and M. Burghard, Small, 2005, 1, 180-192.

156 J. R. Xiao, B. A. Gama and J. W. Gillespie, Int. J. Solids Struct., 2005, 42, 3075-3092.

157 H. Kim, M. Wang, S. K. Lee, J. Kang, J.-D. Nam, L. Ci and J. Suhr, Sci. Rep., 2017, 7, 9512.

158 J. H. Lehman, M. Terrones, E. Mansfield, K. E. Hurst and V. Meunier, Carbon, 2011, 49, 2581-2602.

159 N. G. Sahoo, S. Rana, J. W. Cho, L. Li and S. H. Chan, Prog. Polym. Sci., 2010, 35, 837-867.

160 T. Kuang, L. Chang, F. Chen, Y. Sheng, D. Fu and X. Peng, Carbon, 2016, 105, 305-313.

161 Z. Wei, M. Kondratenko, L. H. Dao and D. F. Perepichka, J. Am. Chem. Soc., 2006, 128, 3134-3135.

162 M. Bockrath, Science, 2001, 291, 283-285.

163 M. Rother, M. Brohmann, S. Yang, S. B. Grimm, S. P. Schießl, A. Graf and J. Zaumseil, Adv. Electron. Mater., 2017, 3, 1700080.

164 J.-M. Bonard, M. Croci, C. Klinke, R. Kurt, O. Noury and N. Weiss, Carbon, 2002, 40, 1715-1728.

165 B. J. Landi, M. J. Ganter, C. D. Cress, R. A. DiLeo and R. P. Raffaelle, Energy Environ. Sci., 2009, 2, 638.

166 S. Mallakpour and E. Khadem, Chem. Eng. J., 2016, 302, 344-367.

167 M. W. Chik, Z. Hussain, M. Zulkefeli, M. Tripathy, S. Kumar, A. B. A. Majeed and K. Byrappa, Drug Delivery Transl. Res., 2019, 9, 578-594.

168 W. Z. Yuan, Y. Mao, H. Zhao, J. Z. Sun, H. P. Xu, J. K. Jin, Q. Zheng and B. Z. Tang, Macromolecules, 2008, 41, 701-707. 
169 C. A. Dyke and J. M. Tour, J. Phys. Chem. A, 2004, 108, 11151-11159.

170 S. Singh, S. Vardharajula, P. Tiwari, E. Eroğlu, K. Vig, V. Dennis and S. Z. Ali, Int. J. Nanomed., 2012, 7, 5361.

171 K. Zare, V. K. Gupta, O. Moradi, A. S. H. Makhlouf, M. Sillanpää, M. N. Nadagouda, H. Sadegh, R. Shahryarighoshekandi, A. Pal, Z. Wang, I. Tyagi and M. Kazemi, J. Nanostruct. Chem., 2015, 5, 227-236.

172 E. Vázquez and M. Prato, Pure Appl. Chem., 2010, 82, 853-861.

173 A. Setaro, M. Adeli, M. Glaeske, D. Przyrembel, T. Bisswanger, G. Gordeev, F. Maschietto, A. Faghani, B. Paulus, M. Weinelt, R. Arenal, R. Haag and S. Reich, Nat. Commun., 2017, 8, 14281. 174 P. Bilalis, D. Katsigiannopoulos, A. Avgeropoulos and G. Sakellariou, RSC Adv., 2014, 4, 2911-2934.

175 Y. Zhou, Y. Fang and R. Ramasamy, Sensors, 2019, 19, 392. 176 T. Morishita, M. Matsushita, Y. Katagiri and K. Fukumori, Carbon, 2009, 47, 2716-2726.

177 M. S. Ata, R. Poon, A. M. Syed, J. Milne and I. Zhitomirsky, Carbon, 2018, 130, 584-598.

178 N. Alzate-Carvajal, L. M. Bolivar-Pineda, V. Meza-Laguna, V. A. Basiuk, E. V. Basiuk and E. A. Baranova, ChemElectroChem, 2020, 7, 428-436.

179 G. Liu, W. Jin and N. Xu, Chem. Soc. Rev., 2015, 44, 5016-5030.

180 N. N. Anh, N. Van Chuc, B. H. Thang, P. Van Nhat, N. Hao, D. D. Phuong, P. N. Minh, T. Subramani, N. Fukata and P. Van Trinh, Global Challenges, 2020, 2000010.

181 G. K. Dalapati, S. Masudy-Panah, R. S. Moakhar, S. Chakrabortty, S. Ghosh, A. Kushwaha, R. Katal, C. S. Chua, G. Xiao, S. Tripathy and S. Ramakrishna, Global Challenges, 2020, 4, 1900087.

182 A. K. Geim and K. S. Novoselov, Nat. Mater., 2007, 6, 183-191. 183 A. K. Geim, Science, 2009, 324, 1530-1534.

184 S. Ghosh, I. Calizo, D. Teweldebrhan, E. P. Pokatilov, D. L. Nika, A. A. Balandin, W. Bao, F. Miao and C. N. Lau, Appl. Phys. Lett., 2008, 92, 151911.

185 A. A. Balandin, S. Ghosh, W. Bao, I. Calizo, D. Teweldebrhan, F. Miao and C. N. Lau, Nano Lett., 2008, 8, 902-907.

186 K. I. Bolotin, K. J. Sikes, Z. Jiang, M. Klima, G. Fudenberg, J. Hone, P. Kim and H. L. Stormer, Solid State Commun., 2008, 146, 351-355.

187 N. Baig, A.-N. Kawde and M. Ibrahim, Mater. Adv., 2020, 1, 783-793, DOI: 10.1039/D0MA00322K.

188 D. Konios, M. M. Stylianakis, E. Stratakis and E. Kymakis, J. Colloid Interface Sci., 2014, 430, 108-112.

189 S. Stankovich, D. A. Dikin, R. D. Piner, K. A. Kohlhaas, A. Kleinhammes, Y. Jia, Y. Wu, S. T. Nguyen and R. S. Ruoff, Carbon, 2007, 45, 1558-1565.

190 J. Chen, Y. Zhang, M. Zhang, B. Yao, Y. Li, L. Huang, C. Li and G. Shi, Chem. Sci., 2016, 7, 1874-1881.

191 M. Pumera, Electrochem. Commun., 2013, 36, 14-18.

192 F. Perreault, A. Fonseca de Faria and M. Elimelech, Chem. Soc. Rev., 2015, 44, 5861-5896.

193 A. Pulido, P. Concepción, M. Boronat, C. Botas, P. Alvarez, R. Menendez and A. Corma, J. Mater. Chem., 2012, 22, 51-56.
194 N. Baig and T. A. Saleh, Microchim. Acta, 2018, 185, 283.

195 A. T. Smith, A. M. LaChance, S. Zeng, B. Liu and L. Sun, Nano Mater. Sci., 2019, 1, 31-47.

196 S. Pei and H.-M. Cheng, Carbon, 2012, 50, 3210-3228.

197 N. Baig and A.-N. Kawde, RSC Adv., 2016, 6, 80756-80765.

198 K. K. H. De Silva, H. H. Huang, R. K. Joshi and M. Yoshimura, Carbon, 2017, 119, 190-199.

199 N. Baig, A. Kawde and M. Ibrahim, ChemistrySelect, 2019, 4, 1640-1649.

200 A. T. Smith, A. M. LaChance, S. Zeng, B. Liu and L. Sun, Nano Mater. Sci., 2019, 1, 31-47.

201 B. Wang, T. Ruan, Y. Chen, F. Jin, L. Peng, Y. Zhou, D. Wang and S. Dou, Energy Storage Mater., 2020, 24, 22-51.

202 W. Lv, Z. Li, Y. Deng, Q.-H. Yang and F. Kang, Energy Storage Mater., 2016, 2, 107-138.

203 G. Li, Y. Xia, Y. Tian, Y. Wu, J. Liu, Q. He and D. Chen, J. Electrochem. Soc., 2019, 166, B881-B895.

204 N. Baig and A.-N. Kawde, Anal. Methods, 2015, 7, 9535-9541.

205 C. I. L. Justino, A. R. Gomes, A. C. Freitas, A. C. Duarte and T. A. P. Rocha-Santos, TrAC, Trends Anal. Chem., 2017, 91, 53-66.

206 Q. He, S. Wu, Z. Yin and H. Zhang, Chem. Sci., 2012, 3, 1764.

207 H. Yoon, J. Nah, H. Kim, S. Ko, M. Sharifuzzaman, S. C. Barman, X. Xuan, J. Kim and J. Y. Park, Sens. Actuators, $B, 2020,311,127866$.

208 T.-H. Han, Y. Lee, M.-R. Choi, S.-H. Woo, S.-H. Bae, B. H. Hong, J.-H. Ahn and T.-W. Lee, Nat. Photonics, 2012, 6, 105-110.

209 M. Xu, J. Qi, F. Li, X. Liao, S. Liu and Y. Zhang, RSC Adv., 2017, 7, 30506-30512.

210 A. Kaidarova, N. Alsharif, B. N. M. Oliveira, M. Marengo, N. R. Geraldi, C. M. Duarte and J. Kosel, Global Challenges, 2020, 4, 2000001.

211 V. H. R. Souza, M. M. Oliveira and A. J. G. Zarbin, J. Power Sources, 2017, 348, 87-93.

212 Y. Zhong, X. Zhang, Y. He, H. Peng, G. Wang and G. Xin, Adv. Funct. Mater., 2018, 28, 1801998.

213 Mandeep, A. Gulati and R. Kakkar, Chem. Eng. Res. Des., 2020, 153, 21-36.

214 T. A. Saleh, N. Baig, F. I. Alghunaimi and N. W. Aljuryyed, RSC Adv., 2020, 10, 5088-5097.

215 N. Baig, F. I. Alghunaimi, H. S. Dossary and T. A. Saleh, Process Saf. Environ. Prot., 2019, 123, 327-334.

216 N. Baig, Ihsanullah, M. Sajid and T. A. Saleh, J. Environ. Manage., 2019, 244, 370-382.

217 J. Bu, L. Yuan, N. Zhang, D. Liu, Y. Meng and X. Peng, Diamond Relat. Mater., 2020, 101, 107604.

218 V. V. Danilenko, Phys. Solid State, 2004, 46, 595-599.

219 V. N. Mochalin, O. Shenderova, D. Ho and Y. Gogotsi, Nat. Nanotechnol., 2012, 7, 11-23.

220 Y. Zhang, K. Y. Rhee, D. Hui and S.-J. Park, Composites, Part $B, 2018,143,19-27$.

221 T. L. Daulton, M. A. Kirk, R. S. Lewis and L. E. Rehn, Nucl. Instrum. Methods Phys. Res., Sect. B, 2001, 175-177, 12-20. 
222 J.-P. Boudou, P. A. Curmi, F. Jelezko, J. Wrachtrup, P. Aubert, M. Sennour, G. Balasubramanian, R. Reuter, A. Thorel and E. Gaffet, Nanotechnology, 2009, 20, 235602. 223 S. Welz, Y. Gogotsi and M. J. McNallan, J. Appl. Phys., 2003, 93, 4207-4214.

224 F. Fendrych, A. Taylor, L. Peksa, I. Kratochvilova, J. Vlcek, V. Rezacova, V. Petrak, Z. Kluiber, L. Fekete, M. Liehr and M. Nesladek, J. Phys. D: Appl. Phys., 2010, 43, 374018.

225 D. Amans, A.-C. Chenus, G. Ledoux, C. Dujardin, C. Reynaud, O. Sublemontier, K. Masenelli-Varlot and O. Guillois, Diamond Relat. Mater., 2009, 18, 177-180.

226 S. Kumar, M. Nehra, D. Kedia, N. Dilbaghi, K. Tankeshwar and K.-H. Kim, Carbon, 2019, 143, 678-699.

227 D. H. Jariwala, D. Patel and S. Wairkar, Mater. Sci. Eng., C, 2020, 113, 110996.

228 I. Neitzel, V. N. Mochalin and Y. Gogotsi, Nanodiamonds, Elsevier, 2017, pp. 365-390.

229 H. Wang and Y. Cui, Carbon Energy, 2019, 1, 13-18.

230 H. Lee, H. Kim, S. Weon, W. Choi, Y. S. Hwang, J. Seo, C. Lee and J.-H. Kim, Environ. Sci. Technol., 2016, 50, 10134-10142.

231 N. A. Zambianco, T. A. Silva, H. Zanin, O. Fatibello-Filho and B. C. Janegitz, Diamond Relat. Mater., 2019, 98, 107512.

232 H. Lai, M. H. Stenzel and P. Xiao, Int. Mater. Rev., 2020, 65, 189-225.

233 U. T. Uthappa, O. R. Arvind, G. Sriram, D. Losic, H.-Y. Jung, M. Kigga and M. D. Kurkuri, J. Drug Delivery Sci. Technol., 2020, 60, 101993.

234 X. Xu, R. Ray, Y. Gu, H. J. Ploehn, L. Gearheart, K. Raker and W. A. Scrivens, J. Am. Chem. Soc., 2004, 126, 12736-12737.

235 J. Shen, Y. Zhu, X. Yang and C. Li, Chem. Commun., 2012, 48, 3686.

236 X. Wang, Y. Feng, P. Dong and J. Huang, Front. Chem., 2019, 7, 671 .

237 P. Karfa, S. De, K. C. Majhi, R. Madhuri and P. K. Sharma, Comprehensive Nanoscience and Nanotechnology, Elsevier, 2019, vol. 1-5, pp. 123-144.

238 M. Li, T. Chen, J. J. Gooding and J. Liu, ACS Sens., 2019, 4, 1732-1748.

239 P. Devi, S. Saini and K.-H. Kim, Biosens. Bioelectron., 2019, 141, 111158.

240 K. J. Mintz, Y. Zhou and R. M. Leblanc, Nanoscale, 2019, 11, 4634-4652.

241 H. Liu, T. Ye and C. Mao, Angew. Chem., Int. Ed., 2007, 46, 6473-6475.

242 D. Pan, J. Zhang, Z. Li, C. Wu, X. Yan and M. Wu, Chem. Commun., 2010, 46, 3681.

243 R. Liu, D. Wu, S. Liu, K. Koynov, W. Knoll and Q. Li, Angew. Chem., Int. Ed., 2009, 48, 4598-4601.

244 Y. Dong, N. Zhou, X. Lin, J. Lin, Y. Chi and G. Chen, Chem. Mater., 2010, 22, 5895-5899.

245 J. Zhou, C. Booker, R. Li, X. Zhou, T.-K. Sham, X. Sun and Z. Ding, J. Am. Chem. Soc., 2007, 129, 744-745.

246 J. C. G. Esteves da Silva and H. M. R. Gonçalves, TrAC, Trends Anal. Chem., 2011, 30, 1327-1336.
247 Y. Wang and A. Hu, J. Mater. Chem. C, 2014, 2, 6921.

248 S. N. Baker and G. A. Baker, Angew. Chem., Int. Ed., 2010, 49, 6726-6744.

249 Y. Li and S. Han, Microchem. J., 2020, 154, 104638.

250 J. Jiang, Y. He, S. Li and H. Cui, Chem. Commun., 2012, 48, 9634.

251 Z.-F. Pu, Q.-L. Wen, Y.-J. Yang, X.-M. Cui, J. Ling, P. Liu and Q.-E. Cao, Spectrochim. Acta, Part A, 2020, 229, 117944.

252 L. Cao, X. Wang, M. J. Meziani, F. Lu, H. Wang, P. G. Luo, Y. Lin, B. A. Harruff, L. M. Veca, D. Murray, S.-Y. Xie and Y.-P. Sun, J. Am. Chem. Soc., 2007, 129, 11318-11319.

253 K. Cheng, R. Qi, S. Lan, H. Wang, X. Zheng, C. Liu, D. Jia, L. Cao and D. Wang, Dyes Pigm., 2020, 174, 108106.

254 F. Copur, N. Bekar, E. Zor, S. Alpaydin and H. Bingol, Sens. Actuators, B, 2019, 279, 305-312.

255 Z. Nan, C. Hao, X. Zhang, H. Liu and R. Sun, Spectrochim. Acta, Part A, 2020, 228, 117717.

256 C. Wang, H. Shi, M. Yang, Y. Yan, E. Liu, Z. Ji and J. Fan, J. Photochem. Photobiol., A, 2020, 391, 112374.

257 W. Li, S. Huang, H. Wen, Y. Luo, J. Cheng, Z. Jia, P. Han and W. Xue, Anal. Bioanal. Chem., 2020, 412, 993-1002.

258 X. Tian, H. Peng, Y. Li, C. Yang, Z. Zhou and Y. Wang, Sens. Actuators, B, 2017, 243, 1002-1009.

259 G. Han, J. Zhao, R. Zhang, X. Tian, Z. Liu, A. Wang, R. Liu, B. Liu, M. Han, X. Gao and Z. Zhang, Angew. Chem., Int. Ed., 2019, 58, 7087-7091.

260 H. Li, F.-Q. Shao, H. Huang, J.-J. Feng and A.-J. Wang, Sens. Actuators, B, 2016, 226, 506-511.

261 Y. Sun, S. Zheng, L. Liu, Y. Kong, A. Zhang, K. Xu and C. Han, Nanoscale Res. Lett., 2020, 15, 55.

262 Y. Wei, L. Chen, J. Wang, X. Liu, Y. Yang and S. Yu, Opt. Mater., 2020, 100, 109647.

263 T. Song, Y. Zhao, T. Wang, J. Li, Z. Jiang and P. Yang, J. Fluoresc., 2020, 30, 81-89.

264 G. Huang, Y. Lin, L. Zhang, Z. Yan, Y. Wang and Y. Liu, Sci. Rep., 2019, 9, 19651.

265 X. Wang, L. Cao, F. Lu, M. J. Meziani, H. Li, G. Qi, B. Zhou, B. A. Harruff, F. Kermarrec and Y.-P. Sun, Chem. Commun., 2009, 3774.

266 Y. Li, B.-P. Zhang, J.-X. Zhao, Z.-H. Ge, X.-K. Zhao and L. Zou, Appl. Surf. Sci., 2013, 279, 367-373.

267 Y. Zhang, Y. Zhao, Z. Xu, H. Su, X. Bian, S. Zhang, X. Dong, L. Zeng, T. Zeng, M. Feng, L. Li and V. K. Sharma, Appl. Catal., B, 2020, 262, 118306.

268 L. Zhang, J. Zhang, Y. Xia, M. Xun, H. Chen, X. Liu and X. Yin, Int. J. Mol. Sci., 2020, 21, 1052.

269 Y.-Y. Li, Y. Si, B.-X. Zhou, T. Huang, W.-Q. Huang, W. Hu, A. Pan, X. Fan and G.-F. Huang, Nanoscale, 2020, 12, 3135-3145.

270 N. Karousis, I. Suarez-Martinez, C. P. Ewels and N. Tagmatarchis, Chem. Rev., 2016, 116, 4850-4883.

271 M. Zhang and M. Yudasaka, Carbon Nanostructures, Springer International Publishing, 2016, pp. 77-107.

272 S. Iijima, M. Yudasaka, R. Yamada, S. Bandow, K. Suenaga, F. Kokai and K. Takahashi, Chem. Phys. Lett., 1999, 309, 165-170. 
273 S. Zhu and G. Xu, Nanoscale, 2010, 2, 2538.

274 J. Fan, M. Yudasaka, J. Miyawaki, K. Ajima, K. Murata and S. Iijima, J. Phys. Chem. B, 2006, 110, 1587-1591.

275 Z. Zhang, S. Han, C. Wang, J. Li and G. Xu, Nanomaterials, 2015, 5, 1732-1755.

276 Z. Liu, W. Zhang, W. Qi, W. Gao, S. Hanif, M. Saqib and G. Xu, Chem. Commun., 2015, 51, 4256-4258.

277 T. Ohba, K. Murata, K. Kaneko, W. A. Steele, F. Kokai, K. Takahashi, D. Kasuya, M. Yudasaka and S. Iijima, Nano Lett., 2001, 1, 371-373.

278 D. Ebenezer, M. Jagannatham, M. S. Lahari, P. Bhuwaneshswar and H. Prathap, Diamond Relat. Mater., 2016, 70, 26-32.

279 H. Wang, L. Pan, Y. Liu, Y. Ye and S. Yao, J. Electroanal. Chem., 2020, 862, 113955.

280 T. Yoshitake, Y. Shimakawa, S. Kuroshima, H. Kimura, T. Ichihashi, Y. Kubo, D. Kasuya, K. Takahashi, F. Kokai, M. Yudasaka and S. Iijima, Phys. B, 2002, 323, 124-126.

281 A. Thomas, Nat. Commun., 2020, 11, 4985.

282 R. W. Derlet and T. E. Albertson, West. J. Med., 1986, 145, 493-496.

283 R. Mishra, J. Militky and M. Venkataraman, Nanotechnology in Textiles: Theory and Application, 2018, pp. 311-353.

284 N. Pal, Adv. Colloid Interface Sci., 2020, 280, 102156.

285 M. E. Davis, Nature, 2002, 417, 813-821.

286 S. Polarz and B. Smarsly, J. Nanosci. Nanotechnol., 2002, 2, 581-612.

287 B. Szczęśniak, S. Borysiuk, J. Choma and M. Jaroniec, Mater. Horiz., 2020, 7, 1457-1473.

288 D. Chen, L. Wei, J. Li and Q. Wu, J. Energy Storage, 2020, 30, 101525.

289 L. Zhang and M. Jaroniec, Chem. Soc. Rev., 2020, 49, 6039-6055.

290 Y. Li, H. Cao and J. Yu, ACS Nano, 2018, 12, 4096-4104.

291 Z. Yang, Z. Xie, H. Liu, F. Yan and H. Ju, Adv. Funct. Mater., 2008, 18, 3991-3998.

292 S. Bagheri, M. M. Amini, M. Behbahani and G. Rabiee, Microchem. J., 2019, 145, 460-469.

293 Y. Zhou, G. Quan, Q. Wu, X. Zhang, B. Niu, B. Wu, Y. Huang, X. Pan and C. Wu, Acta Pharm. Sin. B, 2018, 8, 165-177.

294 X. Wang, C. Li, N. Fan, J. Li, Z. He and J. Sun, Mater. Sci. Eng., C, 2017, 78, 370-375.

295 V. Zeleňák, D. Halamová, M. Almáši, L. Žid, A. Zeleňáková and O. Kapusta, Appl. Surf. Sci., 2018, 443, 525-534.

296 Y. Wang, Q. Zhao, N. Han, L. Bai, J. Li, J. Liu, E. Che, L. Hu, Q. Zhang, T. Jiang and S. Wang, Nanomedicine, 2015, 11, 313-327.

297 K. Na and G. A. Somorjai, Catal. Lett., 2015, 145, 193-213.

298 K. Na, M. Choi and R. Ryoo, Microporous Mesoporous Mater., 2013, 166, 3-19.

299 S. Zhang, Z. Zhang, H. Li, Z. Yu, Q. Huang, Z. Qiao, L. Zong, L. Yan, J. Li and J. Kang, Chem. Eng. J., 2020, 383, 123097.

300 A. Mohammadi Zardkhoshoui and S. S. Hosseiny Davarani, Dalton Trans., 2020, 49, 10028-10041.
301 S. Ouyang, P. Li, H. Xu, H. Tong, L. Liu and J. Ye, ACS Appl. Mater. Interfaces, 2014, 6, 22726-22732.

302 T. Xu, H. Zheng and P. Zhang, J. Hazard. Mater., 2020, 388, 121746.

303 W. Luc and F. Jiao, Acc. Chem. Res., 2016, 49, 1351-1358.

304 T. Zhang, M. Paulose, R. Neupane, L. A. Schaffer, D. B. Rana, J. Su, L. Guo and O. K. Varghese, Sol. Energy Mater. Sol. Cells, 2020, 209, 110472.

305 G. Schmid, J. Mater. Chem., 2002, 12, 1231-1238.

306 A. Larsson, G. Abbondanza, W. Linpé, F. Carlà, P. Mousley, C. Hetherington, E. Lundgren and G. S. Harlow, J. Electrochem. Soc., 2020, 167, 122514.

307 P. G. Schiavi, P. Altimari, A. Rubino and F. Pagnanelli, Electrochim. Acta, 2018, 259, 711-722.

308 A. Yadav, M. S. Bobji and S. J. Bull, J. Nanosci. Nanotechnol., 2019, 19, 4254-4259.

309 F. Yang, J. Tang, B. Shao, S. Gao and X. Liu, Nano-Struct. Nano-Objects, 2019, 18, 100287.

310 G. Rajeev, B. Prieto Simon, L. F. Marsal and N. H. Voelcker, Adv. Healthcare Mater., 2018, 7, 1700904.

311 M. R. Benzigar, S. N. Talapaneni, S. Joseph, K. Ramadass, G. Singh, J. Scaranto, U. Ravon, K. Al-Bahily and A. Vinu, Chem. Soc. Rev., 2018, 47, 2680-2721.

312 N. Baig and T. A. Saleh, Composites, Part B, 2019, 173, 106805.

313 N. Baig and T. A. Saleh, Global Challenges, 2019, 3, 1800115.

314 N. Baig and T. A. Saleh, Chem. - Asian J., 2021, 16, 329-341, DOI: 10.1002/asia.202001368.

315 S. Kundu, I. H. Chowdhury and M. K. Naskar, ACS Omega, 2018, 3, 9888-9898.

316 T. A. Saleh, N. Baig, H. A. Othman and A. M. Al Harith, Chem. Eng. J., 2021, 407, 126216.

317 B. Szczęśniak, J. Phuriragpitikhon, J. Choma and M. Jaroniec, J. Mater. Chem. A, 2020, 8, 18464-18491.

318 Z. Bi, Q. Kong, Y. Cao, G. Sun, F. Su, X. Wei, X. Li, A. Ahmad, L. Xie and C.-M. Chen, J. Mater. Chem. A, 2019, 7, 16028-16045.

319 Z. Li, D. Guo, Y. Liu, H. Wang and L. Wang, Chem. Eng. J., 2020, 397, 125418.

320 Z. Li, J. Xu, D. Sun, T. Lin and F. Huang, ACS Appl. Nano Mater., 2020, 3, 1564-1570.

321 A. O. Abo El Naga, S. A. Shaban and F. Y. A. El Kady, J. Taiwan Inst. Chem. Eng., 2018, 93, 363-373.

322 C. Jiao, Y. Wang, M. Li, Q. Wu, C. Wang and Z. Wang, J. Magn. Magn. Mater., 2016, 407, 24-30.

323 M. Anbia and S. Amirmahmoodi, Arabian J. Chem., 2016, 9, S319-S325.

324 H. Wang, J. Jia, P. Song, Q. Wang, D. Li, S. Min, C. Qian, L. Wang, Y. F. Li, C. Ma, T. Wu, J. Yuan, M. Antonietti and G. A. Ozin, Angew. Chem., 2017, 129, 7955-7960.

325 Y. Zhang, H. Sun, Y. Qiu, E. Zhang, T. Ma, G. Gao, C. Cao, Z. Ma and P. Hu, Energy, 2018, 165, 537-548.

326 H. Luo, W.-J. Jiang, Y. Zhang, S. Niu, T. Tang, L.-B. Huang, Y.-Y. Chen, Z. Wei and J.-S. Hu, Carbon, 2018, 128, 97-105.

327 J. Tang, J. Liu, N. L. Torad, T. Kimura and Y. Yamauchi, Nano Today, 2014, 9, 305-323.

328 R. Brilmayer, C. Förster, L. Zhao and A. Andrieu-Brunsen, Curr. Opin. Biotechnol., 2020, 63, 200-209. 
329 O. K. Farha, A. Özgür Yazaydın, I. Eryazici, C. D. Malliakas, B. G. Hauser, M. G. Kanatzidis, S. T. Nguyen, R. Q. Snurr and J. T. Hupp, Nat. Chem., 2010, 2, 944-948.

330 O. K. Farha, I. Eryazici, N. C. Jeong, B. G. Hauser, C. E. Wilmer, A. A. Sarjeant, R. Q. Snurr, S. T. Nguyen, A. Ö. Yazaydın and J. T. Hupp, J. Am. Chem. Soc., 2012, 134, 15016-15021.

331 I. M. Hönicke, I. Senkovska, V. Bon, I. A. Baburin, N. Bönisch, S. Raschke, J. D. Evans and S. Kaskel, Angew. Chem., Int. Ed., 2018, 57, 13780-13783.

332 V. Chernikova, O. Shekhah, Y. Belmabkhout and M. Eddaoudi, ACS Appl. Nano Mater., 2020, 3, 6432-6439.

333 H. A. Patel, S. Hyun Je, J. Park, D. P. Chen, Y. Jung, C. T. Yavuz and A. Coskun, Nat. Commun., 2013, 4, 1357.

334 Y. Zhang, S. Wei, F. Liu, Y. Du, S. Liu, Y. Ji, T. Yokoi, T. Tatsumi and F.-S. Xiao, Nano Today, 2009, 4, 135-142.

335 M. Rose, ChemCatChem, 2014, 6, 1166-1182.

336 N. Wang, Q. Sun and J. Yu, Adv. Mater., 2019, 31, 1803966. 337 C. R. Martin, Science, 1994, 266, 1961-1966.

338 H. Zhao, X. Chen, G. Wang, Y. Qiu and L. Guo, 2D Mater., 2019, 6, 032002, DOI: 10.1088/2053-1583/ab1169.

339 C. Tan, X. Cao, X.-J. Wu, Q. He, J. Yang, X. Zhang, J. Chen, W. Zhao, S. Han, G.-H. Nam, M. Sindoro and H. Zhang, Chem. Rev., 2017, 117, 6225-6331.

340 A. A. F. K. S. Novoselov, A. K. Geim, S. V. Morozov, D. Jiang, Y. Zhang, S. V. Dubonos and I. V. Grigorieva, Science, 2004, 306, 666-669.

341 F. Bai, L. Xu, X. Zhai, X. Chen and W. Yang, Adv. Energy Mater., 2020, 10, 1-19.

342 K. Takeda and K. Shiraishi, Teoriâ ta Metod. Fizičnogo Vihovannâ, 1994, 50, 14916-14922.

343 G. G. Guzmán-Verri and L. C. Lew Yan Voon, Phys. Rev. B: Condens. Matter Mater. Phys., 2007, 76, 075131, DOI: 10.1103/PhysRevB.76.075131.

344 S. Cahangirov, M. Topsakal, E. Aktürk, H. Šahin and S. Ciraci, Phys. Rev. Lett., 2009, 102, 1-4.

345 N. D. Drummond, V. Zólyomi and V. I. Fal'Ko, Phys. Rev. B: Condens. Matter Mater. Phys., 2012, 85, 1-7.

346 Z. Ni, Q. Liu, K. Tang, J. Zheng, J. Zhou, R. Qin, Z. Gao, D. Yu and J. Lu, Nano Lett., 2012, 12, 113-118.

347 C. C. Liu, W. Feng and Y. Yao, Phys. Rev. Lett., 2011, 107, 1-4.

348 F. Liu, C. C. Liu, K. Wu, F. Yang and Y. Yao, Phys. Rev. Lett., 2013, 111, 1-5.

349 C. Xu, G. Luo, Q. Liu, J. Zheng, Z. Zhang, S. Nagase, Z. Gao and J. Lu, Nanoscale, 2012, 4, 3111-3117.

350 J. Zhao, H. Liu, Z. Yu, R. Quhe, S. Zhou, Y. Wang, C. C. Liu, H. Zhong, N. Han, J. Lu, Y. Yao and K. Wu, Prog. Mater. Sci., 2016, 83, 24-151.

351 M. De Crescenzi, P. Castrucci, M. Scarselli, M. Diociaiuti, P. S. Chaudhari, C. Balasubramanian, T. M. Bhave and S. V. Bhoraskar, Appl. Phys. Lett., 2005, 86, 1-3.

352 S. Yamada and H. Fujiki, Jpn. J. Appl. Phys., Part 2, 2006, 45, 18-21.

353 H. Nakano, T. Mitsuoka, M. Harada, K. Horibuchi, H. Nozaki, N. Takahashi, T. Nonaka, Y. Seno and H. Nakamura, Angew. Chem., Int. Ed., 2006, 45, 6303-6306.
354 P. De Padova, C. Quaresima, C. Ottaviani, P. M. Sheverdyaeva, P. Moras, C. Carbone, D. Topwal, B. Olivieri, A. Kara, H. Oughaddou, B. Aufray and G. Le Lay, Appl. Phys. Lett., 2010, 96, 1-4.

355 B. Aufray, A. Kara, Ś. Vizzini, H. Oughaddou, C. Ĺandri, B. Ealet and G. Le-Lay, Appl. Phys. Lett., 2010, 96, 1-4.

356 C. Léandri, H. Oughaddou, B. Aufray, J. M. Gay, G. Le Lay, A. Ranguis and Y. Garreau, Surf. Sci., 2007, 601, 262-267.

357 P. Vogt, P. De Padova, C. Quaresima, J. Avila, E. Frantzeskakis, M. C. Asensio, A. Resta, B. Ealet and G. Le Lay, Phys. Rev. Lett., 2012, 108, 155501.

358 A. Fleurence, R. Friedlein, T. Ozaki, H. Kawai, Y. Wang and Y. Yamada-Takamura, Phys. Rev. Lett., 2012, 108, 1-5.

359 L. Meng, Y. Wang, L. Zhang, S. Du, R. Wu, L. Li, Y. Zhang, G. Li, H. Zhou, W. A. Hofer and H. J. Gao, Nano Lett., 2013, 13, 685-690.

360 T. Aizawa, S. Suehara and S. Otani, J. Phys. Chem. C, 2014, 118, 23049-23057.

361 D. Chiappe, E. Scalise, E. Cinquanta, C. Grazianetti, B. Van Den Broek, M. Fanciulli, M. Houssa and A. Molle, Adv. Mater., 2014, 26, 2096-2101.

362 B. Feng, Z. Ding, S. Meng, Y. Yao, X. He, P. Cheng, L. Chen and K. Wu, Nano Lett., 2012, 12, 3507-3511.

363 D. Chiappe, C. Grazianetti, G. Tallarida, M. Fanciulli and A. Molle, Adv. Mater., 2012, 24, 5088-5093.

364 P. De Padova, O. Kubo, B. Olivieri, C. Quaresima, T. Nakayama, M. Aono and G. Le Lay, Nano Lett., 2012, 12, 5500-5503.

365 L. Tao, E. Cinquanta, D. Chiappe, C. Grazianetti, M. Fanciulli, M. Dubey, A. Molle and D. Akinwande, Nat. Nanotechnol., 2015, 10, 227-231.

366 J. Liu, Y. Yang, P. Lyu, P. Nachtigall and Y. Xu, Adv. Mater., 2018, 30, 1-7.

367 M. Naguib, V. N. Mochalin, M. W. Barsoum and Y. Gogotsi, Adv. Mater., 2014, 26, 992-1005.

368 M. Ghidiu, M. R. Lukatskaya, M. Q. Zhao, Y. Gogotsi and M. W. Barsoum, Nature, 2014, 516, 78-81.

369 X. Sang, Y. Xie, M. W. Lin, M. Alhabeb, K. L. Van Aken, Y. Gogotsi, P. R. C. Kent, K. Xiao and R. R. Unocic, ACS Nano, 2016, 10, 9193-9200.

370 M. Naguib, M. Kurtoglu, V. Presser, J. Lu, J. Niu, M. Heon, L. Hultman, Y. Gogotsi and M. W. Barsoum, Adv. Mater., 2011, 23, 4248-4253.

371 J. Pang, R. G. Mendes, A. Bachmatiuk, L. Zhao, H. Q. Ta, T. Gemming, H. Liu, Z. Liu and M. H. Rummeli, Chem. Soc. Rev., 2019, 48, 72-133.

372 Z. Li and Y. Wu, Small, 2019, 15, 1-10.

373 J. Ran, G. Gao, F. T. Li, T. Y. Ma, A. Du and S. Z. Qiao, Nat. Commun., 2017, 8, 13907, DOI: 10.1038/ncomms13907.

374 Z. W. Seh, K. D. Fredrickson, B. Anasori, J. Kibsgaard, A. L. Strickler, M. R. Lukatskaya, Y. Gogotsi, T. F. Jaramillo and A. Vojvodic, ACS Energy Lett., 2016, 1, 589-594.

375 A. Kumar and A. Bhan, Chem. Eng. Sci., 2019, 197, 371-378. 376 D. Wang, Z. Wang, L. Wang, L. Hu and J. Jin, Nanoscale, 2015, 7, 17649-17652.

377 J. Abraham, K. S. Vasu, C. D. Williams, K. Gopinadhan, Y. Su, C. T. Cherian, J. Dix, E. Prestat, S. J. Haigh, 
I. V. Grigorieva, P. Carbone, A. K. Geim and R. R. Nair, Nat. Nanotechnol., 2017, 12, 546-550.

378 G. Reina, J. M. González-Domínguez, A. Criado, E. Vázquez, A. Bianco and M. Prato, Chem. Soc. Rev., 2017, 46, 4400-4416.

379 Y. Zhang, H. Jiang, Y. Lin, H. Liu, Q. He, C. Wu, T. Duan and L. Song, Adv. Mater. Interfaces, 2018, 5, 1-9.

380 T. Y. Ma, J. L. Cao, M. Jaroniec and S. Z. Qiao, Angew. Chem., 2016, 128, 1150-1154.

381 M. Yu, S. Zhou, Z. Wang, J. Zhao and J. Qiu, Nano Energy, 2018, 44, 181-190.

382 N. Hao, Y. Wei, J. Wang, Z. Wang, Z. Zhu, S. Zhao, M. Han and X. Huang, RSC Adv., 2018, 8, 20576-20584.

383 L. Zhao, B. Dong, S. Li, L. Zhou, L. Lai, Z. Wang, S. Zhao, M. Han, K. Gao, M. Lu, X. Xie, B. Chen, Z. Liu, X. Wang, H. Zhang, H. Li, J. Liu, H. Zhang, X. Huang and W. Huang, ACS Nano, 2017, 11, 5800-5807.

384 B. Lyu, Y. Choi, H. Jing, C. Qian, H. Kang, S. Lee and J. H. Cho, Adv. Mater., 2020, 32, 2-9.

385 L. Li, Y. Yu, G. J. Ye, Q. Ge, X. Ou, H. Wu, D. Feng, X. H. Chen and Y. Zhang, Nat. Nanotechnol., 2014, 9, 372-377.

386 M. Buscema, D. J. Groenendijk, S. I. Blanter, G. A. Steele, H. S. J. Van Der Zant and A. Castellanos-Gomez, Nano Lett., 2014, 14, 3347-3352.

387 G. Slotman, A. Rudenko, E. Van Veen, M. I. Katsnelson, R. Roldán and S. Yuan, Phys. Rev. B, 2018, 98, 1-7.

388 C. Hao, B. Yang, F. Wen, J. Xiang, L. Li, W. Wang, Z. Zeng, B. Xu, Z. Zhao, Z. Liu and Y. Tian, Adv. Mater., 2016, 28, 3194-3201.

389 C. M. Park and H. J. Sohn, Adv. Mater., 2007, 19, 2465-2468.

390 M. Pumera and Z. Sofer, Adv. Mater., 2017, 29, 1605299, DOI: 10.1002/adma.201605299.

391 S. Zhang, M. Xie, F. Li, Z. Yan, Y. Li, E. Kan, W. Liu, Z. Chen and H. Zeng, Angew. Chem., Int. Ed., 2016, 55, 1666-1669.

392 M. Wu, H. Fu, L. Zhou, K. Yao and X. C. Zeng, Nano Lett., 2015, 15, 3557-3562.

393 P. Ares, F. Aguilar-Galindo, D. Rodríguez-San-Miguel, D. A. Aldave, S. Díaz-Tendero, M. Alcamí, F. Martín, J. Gómez-Herrero and F. Zamora, Adv. Mater., 2016, 28, 6332-6336.

394 Y. Wang and Y. Ding, Nanoscale Res. Lett., 2015, 10, 1-10. 395 J. Ji, X. Song, J. Liu, Z. Yan, C. Huo, S. Zhang, M. Su, L. Liao, W. Wang, Z. Ni, Y. Hao and H. Zeng, Nat. Commun., 2016, 7, 1-9.

396 H. Liu, A. T. Neal, Z. Zhu, Z. Luo, X. Xu, D. Tománek and P. D. Ye, ACS Nano, 2014, 8, 4033-4041.

397 A. Favron, E. Gaufrès, F. Fossard, A. L. Phaneuf-Laheureux, N. Y. W. Tang, P. L. Lévesque, A. Loiseau, R. Leonelli, S. Francoeur and R. Martel, Nat. Mater., 2015, 14, 826-832.

398 G. Abellán, S. Wild, V. Lloret, N. Scheuschner, R. Gillen, U. Mundloch, J. Maultzsch, M. Varela, F. Hauke and A. Hirsch, J. Am. Chem. Soc., 2017, 139, 10432-10440.

399 C. Gibaja, D. Rodriguez-San-Miguel, P. Ares, J. GómezHerrero, M. Varela, R. Gillen, J. Maultzsch, F. Hauke,
A. Hirsch, G. Abellán and F. Zamora, Angew. Chem., 2016, 128, 14557-14561.

400 O. Ü. Aktürk, V. O. Özçelik and S. Ciraci, Phys. Rev. B: Condens. Matter Mater. Phys., 2015, 91, 1-10.

401 S. K. Gupta, Y. Sonvane, G. Wang and R. Pandey, Chem. Phys. Lett., 2015, 641, 169-172.

402 G. Wang, R. Pandey and S. P. Karna, ACS Appl. Mater. Interfaces, 2015, 7, 11490-11496.

403 S. Zhang, Z. Yan, Y. Li, Z. Chen and H. Zeng, Angew. Chem., 2015, 127, 3155-3158.

404 C. Kamal and M. Ezawa, Phys. Rev. B: Condens. Matter Mater. Phys., 2015, 91, 1-10.

405 Z. Zhang, J. Xie, D. Yang, Y. Wang, M. Si and D. Xue, Appl. Phys. Express, 2015, 8, 055201, DOI: 10.7567/ APEX.8.055201.

406 M. S. Khan, A. Srivastava and R. Pandey, RSC Adv., 2016, 6, 72634-72642.

407 M. S. Khan, V. Ranjan and A. Srivastava, Proc. - 2015 IEEE Int. Symp. Nanoelectron. Inf. Syst. iNIS 2015, 2016, 248-251.

408 S. Guo, Y. Zhang, Y. Ge, S. Zhang, H. Zeng and H. Zhang, Adv. Mater., 2019, 31, 1-19.

409 Y. Liu, T. Wang, J. Robertson, J. Luo, Y. Guo and D. Liu, J. Phys. Chem. C, 2020, 124, 7441-7448.

410 G. Abellán, P. Ares, S. Wild, E. Nuin, C. Neiss, D. R. S. Miguel, P. Segovia, C. Gibaja, E. G. Michel, A. Görling, F. Hauke, J. Gómez-Herrero, A. Hirsch and F. Zamora, Angew. Chem., Int. Ed., 2017, 56, 14389-14394.

411 S. Wolff, R. Gillen, M. Assebban, G. Abellán and J. Maultzsch, Phys. Rev. Lett., 2020, 124, 126101.

412 T. García-Mendiola, C. Gutiérrez-Sánchez, C. Gibaja, I. Torres, C. Busó-Rogero, F. Pariente, J. Solera, Z. Razavifar, J. J. Palacios, F. Zamora and E. Lorenzo, ACS Appl. Nano Mater., 2020, 3, 3625-3633.

413 T. Xue, W. Liang, Y. Li, Y. Sun, Y. Xiang, Y. Zhang, Z. Dai, Y. Duo, L. Wu, K. Qi, B. N. Shivananju, L. Zhang, X. Cui, H. Zhang and Q. Bao, Nat. Commun., 2019, 10, 1-9.

414 Z. Wang, Z. Wang, Z. Wang, M. Zhao, Y. Zhou, B. Zhao, Y. Miao, P. Liu, Y. Hao, H. Wang, B. Xu, Y. Wu and S. Yin, $2 D$ Mater., 2019, 6, 045017, DOI: 10.1088/2053-1583/ ab277d.

415 E. Martínez-Periñán, M. P. Down, C. Gibaja, E. Lorenzo, F. Zamora and C. E. Banks, Adv. Energy Mater., 2018, 8, 1-8.

416 Y. H. Luo, C. Chen, C. He, Y. Y. Zhu, D. L. Hong, X. T. He, P. J. An, H. S. Wu and B. W. Sun, ACS Appl. Mater. Interfaces, 2018, 10, 28860-28867.

417 A. Abhervé, S. Mañas-Valero, M. Clemente-León and E. Coronado, Chem. Sci., 2015, 6, 4665-4673.

418 H. S. Wang, J. Li, J. Y. Li, K. Wang, Y. Ding and X. H. Xia, NPG Asia Mater., 2017, 9, 1-9.

419 W. J. Song, Talanta, 2017, 170, 74-80.

420 Y. Ding, Y. P. Chen, X. Zhang, L. Chen, Z. Dong, H. L. Jiang, H. Xu and H. C. Zhou, J. Am. Chem. Soc., 2017, 139, 9136-9139.

421 T. Kambe, R. Sakamoto, K. Hoshiko, K. Takada, M. Miyachi, J. H. Ryu, S. Sasaki, J. Kim, K. Nakazato, M. Takata and H. Nishihara, J. Am. Chem. Soc., 2013, 135, 2462-2465. 
422 L. Cao, Z. Lin, F. Peng, W. Wang, R. Huang, C. Wang, J. Yan, J. Liang, Z. Zhang, T. Zhang, L. Long, J. Sun and W. Lin, Angew. Chem., 2016, 128, 5046-5050.

423 T. Rodenas, I. Luz, G. Prieto, B. Seoane, H. Miro, A. Corma, F. Kapteijn, F. X. Llabrés I Xamena and J. Gascon, Nat. Mater., 2015, 14, 48-55.

424 S. C. Junggeburth, L. Diehl, S. Werner, V. Duppel, W. Sigle and B. V. Lotsch, J. Am. Chem. Soc., 2013, 135, 6157-6164.

425 Y. Zheng, S. Zheng, Y. Xu, H. Xue, C. Liu and H. Pang, Chem. Eng. J., 2019, 373, 1319-1328.

426 Y. Wang, Y. Liu, H. Wang, W. Liu, Y. Li, J. Zhang, H. Hou and J. Yang, ACS Appl. Energy Mater., 2019, 2, 2063-2071.

427 C. Li, X. Hu, W. Tong, W. Yan, X. Lou, M. Shen and B. Hu, ACS Appl. Mater. Interfaces, 2017, 9, 29829-29838.

428 A. J. Clough, J. W. Yoo, M. H. Mecklenburg and S. C. Marinescu, J. Am. Chem. Soc., 2015, 137, 118-121.

429 R. Dong, M. Pfeffermann, H. Liang, Z. Zheng, X. Zhu, J. Zhang and X. Feng, Angew. Chem., Int. Ed., 2015, 54, 12058-12063.

430 B. C. Patra, S. Khilari, R. N. Manna, S. Mondal, D. Pradhan, A. Pradhan and A. Bhaumik, ACS Catal., 2017, 7, 6120-6127.

431 Y. Xu, B. Li, S. Zheng, P. Wu, J. Zhan, H. Xue, Q. Xu and H. Pang, J. Mater. Chem. A, 2018, 6, 22070-22076.

432 G. Hai, X. Jia, K. Zhang, X. Liu, Z. Wu and G. Wang, Nano Energy, 2018, 44, 345-352.

433 M. Jian, H. Liu, T. Williams, J. Ma, H. Wang and X. Zhang, Chem. Commun., 2017, 53, 13161-13164.

434 E. M. Miner, T. Fukushima, D. Sheberla, L. Sun, Y. Surendranath and M. Dinca, Nat. Commun., 2016, 7, 1-7.

435 K. Zhao, S. Liu, G. Ye, X. Wei, Y. Su, W. Zhu, Z. Zhou and Z. He, ChemSusChem, 2020, 13, 1556-1567.

436 N. Kornienko, Y. Zhao, C. S. Kley, C. Zhu, D. Kim, S. Lin, C. J. Chang, O. M. Yaghi and P. Yang, J. Am. Chem. Soc., 2015, 137, 14129-14135.

437 S. He, Y. Chen, Z. Zhang, B. Ni, W. He and X. Wang, Chem. Sci., 2016, 7, 7101-7105.

438 H. Xu, J. Gao, X. Qian, J. Wang, H. He, Y. Cui, Y. Yang, Z. Wang and G. Qian, J. Mater. Chem. A, 2016, 4, 10900-10905.

439 W. P. Lustig, S. Mukherjee, N. D. Rudd, A. V. Desai, J. Li and S. K. Ghosh, Chem. Soc. Rev., 2017, 46, 3242-3285.

440 L. E. Kreno, K. Leong, O. K. Farha, M. Allendorf, R. P. Van Duyne and J. T. Hupp, Chem. Rev., 2012, 112, 1105-1125.

441 M. Zhao, Y. Wang, Q. Ma, Y. Huang, X. Zhang, J. Ping, Z. Zhang, Q. Lu, Y. Yu, H. Xu, Y. Zhao and H. Zhang, Adv. Mater., 2015, 27, 7372-7378.

442 H. S. Wang, H. L. Liu, K. Wang, Y. Ding, J. J. Xu, X. H. Xia and H. Y. Chen, Anal. Chem., 2017, 89, 11366-11371.

443 Y. Zhang, Y. Luo, J. Tian, A. M. Asiri, A. O. Al-Youbi and X. Sun, PLoS One, 2012, 7, e30426, DOI: 10.1371/ journal.pone.0030426.

444 Y. Y. Fan, Z. L. Mou, M. Wang, J. Li, J. Zhang, F. Q. Dang and Z. Q. Zhang, Anal. Chem., 2018, 90, 13708-13713.

445 C. He, K. Lu and W. Lin, J. Am. Chem. Soc., 2014, 136, 12253-12256.
446 Y. Peng, Y. Li, Y. Ban, H. Jin, W. Jiao, X. Liu and W. Yang, Science, 2014, 346, 1356-1359, DOI: 10.1126/science.1254227.

447 Y. Peng, Y. Li, Y. Ban and W. Yang, Angew. Chem., 2017, 129, 9889-9893.

448 S. Jiang, X. Shi, F. Sun and G. Zhu, Chem. - Asian J., 2020, 3, 1-9.

449 Y. Cheng, X. Wang, C. Jia, Y. Wang, L. Zhai, Q. Wang and D. Zhao, J. Membr. Sci., 2017, 539, 213-223.

450 M. Shete, P. Kumar, J. E. Bachman, X. Ma, Z. P. Smith, W. Xu, K. A. Mkhoyan, J. R. Long and M. Tsapatsis, J. Membr. Sci., 2018, 549, 312-320.

451 Z. Kang, Y. Peng, Z. Hu, Y. Qian, C. Chi, L. Y. Yeo, L. Tee and D. Zhao, J. Mater. Chem. A, 2015, 3, 20801-20810.

452 Y. Yang, K. Goh, R. Wang and T. H. Bae, Chem. Commun., 2017, 53, 4254-4257.

453 H. L. Liu, Y. J. Chang, T. Fan and Z. Y. Gu, Chem. Commun., 2016, 52, 12984-12987.

454 M. H. Pham, G. T. Vuong, F. G. Fontaine and T. O. Do, Cryst. Growth Des., 2012, 12, 3091-3095.

455 R. Chen, J. Yao, Q. Gu, S. Smeets, C. Baerlocher, H. Gu, D. Zhu, W. Morris, O. M. Yaghi and H. Wang, Chem. Commun., 2013, 49, 9500-9502.

456 R. Kumar, K. Jayaramulu, T. K. Maji and C. N. R. Rao, Dalton Trans., 2014, 43, 7383-7386.

457 J. Guo, Y. Zhang, Y. Zhu, C. Long, M. Zhao, M. He, X. Zhang, J. Lv, B. Han and Z. Tang, Angew. Chem., Int. Ed., 2018, 57, 6873-6877.

458 V. Galstyan, M. Bhandari, V. Sberveglieri, G. Sberveglieri and E. Comini, Chemosensors, 2018, 6, 16.

459 M. F. Baran, H. Acay and C. Keskin, Global Challenges, 2020, 4, 1900104.

460 G. Maduraiveeran, M. Sasidharan and W. Jin, Prog. Mater. Sci., 2019, 106, 100574.

461 J. Wu, X. Wang, Q. Wang, Z. Lou, S. Li, Y. Zhu, L. Qin and H. Wei, Chem. Soc. Rev., 2019, 48, 1004-1076.

462 H. Bin Wu, J. S. Chen, H. H. Hng and X. Wen (David) Lou, Nanoscale, 2012, 4, 2526.

463 W. Zhang, J. Ai, Y. Lei, Y. Li, C. Lai and J. Xie, Solid State Ionics, 2020, 344, 115132.

464 J. Ying, C. Wan, C. Jiang and Y. Li, J. Power Sources, 2001, 99, 78-84.

465 B. Qu, L. Hu, Q. Li, Y. Wang, L. Chen and T. Wang, ACS Appl. Mater. Interfaces, 2014, 6, 731-736.

466 P.-F. Wang, Y. You, Y.-X. Yin and Y.-G. Guo, Adv. Energy Mater., 2018, 8, 1701912.

467 Y. Fang, X.-Y. Yu and X. W. (David) Lou, Matter, 2019, 1, 90-114.

468 T. Wang, D. Su, D. Shanmukaraj, T. Rojo, M. Armand and G. Wang, Electrochem. Energy Rev., 2018, 1, 200-237.

469 Q. Liu, Z. Hu, M. Chen, C. Zou, H. Jin, S. Wang, S. Chou and S. Dou, Small, 2019, 15, 1805381.

470 Y. Huang, Y. Zheng, X. Li, F. Adams, W. Luo, Y. Huang and L. Hu, ACS Energy Lett., 2018, 3, 1604-1612.

471 J. Ye, G. Xia, Z. Zheng and C. Hu, Int. J. Hydrogen Energy, 2020, 45, 9969-9978.

472 F. Zhang, J. Zhu, D. Zhang, U. Schwingenschlögl and H. N. Alshareef, Nano Lett., 2017, 17, 1302-1311. 
473 L. Liang, Y. Xu, C. Wang, L. Wen, Y. Fang, Y. Mi, M. Zhou, H. Zhao and Y. Lei, Energy Environ. Sci., 2015, 8, 2954-2962.

474 L. Shen, Q. Che, H. Li and X. Zhang, Adv. Funct. Mater., 2014, 24, 2630-2637.

475 D. Kundu, B. D. Adams, V. Duffort, S. H. Vajargah and L. F. Nazar, Nat. Energy, 2016, 1, 16119.

476 J. Ha, Y. Kim and J. Choi, ChemSusChem, 2020, 13, 419-425.

477 Z. Kang, C. Wu, L. Dong, W. Liu, J. Mou, J. Zhang, Z. Chang, B. Jiang, G. Wang, F. Kang and C. Xu, ACS Sustainable Chem. Eng., 2019, 7, 3364-3371.

478 S. Lu, H. Wang, J. Zhou, X. Wu and W. Qin, Nanoscale, 2017, 9, 1184-1192.

479 J.-W. Lee, H.-S. Shin, C.-W. Lee and K.-N. Jung, Nanoscale Res. Lett., 2016, 11, 45.

480 J. Han, J. Qin, L. Guo, K. Qin, N. Zhao, C. Shi, E. Liu, F. He, L. Ma and C. He, Appl. Surf. Sci., 2018, 427, 670-679.

481 Z. Zhang, X. Zhao and J. Li, ChemNanoMat, 2016, 2, 196-200.

482 Y. Xiong, J. Qian, Y. Cao, X. Ai and H. Yang, ACS Appl. Mater. Interfaces, 2016, 8, 16684-16689.

483 X. Wang, K. Cao, Y. Wang and L. Jiao, Small, 2017, 13, 1700873.

484 X. Li, X. Hao, A. Abudula and G. Guan, J. Mater. Chem. A, 2016, 4, 11973-12000.

485 Y. Yan, B. Y. Xia, B. Zhao and X. Wang, J. Mater. Chem. A, 2016, 4, 17587-17603.

486 I. Roger, M. A. Shipman and M. D. Symes, Nat. Rev. Chem., 2017, 1, 0003.

487 X. Zou and Y. Zhang, Chem. Soc. Rev., 2015, 44, 5148-5180.

488 X. Yang, H. Li, A.-Y. Lu, S. Min, Z. Idriss, M. N. Hedhili, K.-W. Huang, H. Idriss and L.-J. Li, Nano Energy, 2016, 25, $42-50$.

489 A. G. Tamirat, J. Rick, A. A. Dubale, W.-N. Su and B.-J. Hwang, Nanoscale Horiz., 2016, 1, 243-267.

490 Y. Yang, S. Niu, D. Han, T. Liu, G. Wang and Y. Li, Adv. Energy Mater., 2017, 7, 1700555.

491 T. Zhang, M. Paulose, R. Neupane, L. A. Schaffer, D. B. Rana, J. Su, L. Guo and O. K. Varghese, Sol. Energy Mater. Sol. Cells, 2020, 209, 110472.

492 N. Nasori, T. Dai, X. Jia, A. Rubiyanto, D. Cao, S. Qu, Z. Wang, Z. Wang and Y. Lei, J. Semicond., 2019, 40, 052701.

493 Z. Yu, H. Liu, M. Zhu, Y. Li and W. Li, Small, 2019, 1903378.

494 V. Sharma, M. Prasad, P. Ilaiyaraja, C. Sudakar and S. Jadkar, Int. J. Hydrogen Energy, 2019, 44, 11459-11471.

495 X. Li, C. Gao, H. Duan, B. Lu, Y. Wang, L. Chen, Z. Zhang, X. Pan and E. Xie, Small, 2013, 9, 2005-2011.

496 M. Tayebi and B.-K. Lee, Renewable Sustainable Energy Rev., 2019, 111, 332-343.

497 J. Guo, Y. Li, B. Jiang, H. Gao, T. Wang, P. Sun, F. Liu, X. Yan, X. Liang, Y. Gao, J. Zhao and G. Lu, Sens. Actuators, $B, 2020,310,127780$.

498 X. Chen and S. S. Mao, Chem. Rev., 2007, 107, 2891-2959.
499 K. Wetchakun, T. Samerjai, N. Tamaekong, C. Liewhiran, C. Siriwong, V. Kruefu, A. Wisitsoraat, A. Tuantranont and S. Phanichphant, Sens. Actuators, B, 2011, 160, 580-591.

500 J. M. George, A. Antony and B. Mathew, Microchim. Acta, 2018, 185, 358.

501 A.-N. Kawde, M. Aziz, N. Baig and Y. Temerk, J. Electroanal. Chem., 2015, 740, 68-74.

502 R. Narayanan and M. A. El-Sayed, Nano Lett., 2004, 4, 1343-1348.

503 X. Xie, Y. Li, Z.-Q. Liu, M. Haruta and W. Shen, Nature, 2009, 458, 746-749.

504 K. M. Bratlie, H. Lee, K. Komvopoulos, P. Yang and G. A. Somorjai, Nano Lett., 2007, 7, 3097-3101.

505 Z. Sun, T. Liao, L. Sheng, L. Kou, J. H. Kim and S. X. Dou, Chem. - Eur. J., 2016, 22, 11357-11364.

506 Q. Liang, X. Zou, H. Chen, M. Fan and G.-D. Li, Sens. Actuators, B, 2020, 304, 127241.

507 S. Ozkan, N. T. Nguyen, I. Hwang, A. Mazare and P. Schmuki, Small, 2017, 13, 1603821.

508 S. Cheon, W. W. Lee, W. Il Park, J.-Y. Jung, J.-H. Choi, D.-G. Choi, S. Jeon, J. Jeong and J. Lee, J. Ind. Eng. Chem., 2020, 81, 385-392.

509 G. A. Cerrón-Calle, A. J. Aranda-Aguirre, C. Luyo, S. GarciaSegura and H. Alarcón, Chemosphere, 2019, 219, 296-304.

510 X. Feng, Y. Huang, C. Li, X. Chen, S. Zhou, X. Gao and C. Chen, Chem. Eng. J., 2019, 368, 51-60.

511 L. Peng, P. Xiong, L. Ma, Y. Yuan, Y. Zhu, D. Chen, X. Luo, J. Lu, K. Amine and G. Yu, Nat. Commun., 2017, 8, 15139.

512 K. Xu, S. Ma, Y. Shen, Q. Ren, J. Yang, X. Chen and J. Hu, Chem. Eng. J., 2019, 369, 363-369.

513 Y. Xie, C. Chen, X. Lu, F. Luo, C. Wang, A. Alsaedi and T. Hayat, J. Hazard. Mater., 2019, 379, 120786.

514 S. M. Saleh, Spectrochim. Acta, Part A, 2019, 211, 141-147.

515 M. Guzman, M. Estrada, S. Miridonov and A. Simakov, Microporous Mesoporous Mater., 2019, 278, 241-250.

516 X. Zhang, W. Lan, J. Xu, Y. Luo, J. Pan, C. Liao, L. Yang, W. Tan and X. Huang, Sens. Actuators, B, 2019, 289, 144-152.

517 M. Wang, Y. Huang, Y. Zhu, N. Zhang, J. Zhang, X. Qin and H. Zhang, Electrochim. Acta, 2020, 335, 135694.

518 J. Feng and Y. Yin, Adv. Mater., 2019, 31, 1802349.

519 Z. Wang, L. Zhou and X. W. David Lou, Adv. Mater., 2012, 24, 1903-1911.

520 X. W. Lou, Y. Wang, C. Yuan, J. Y. Lee and L. A. Archer, Adv. Mater., 2006, 18, 2325-2329.

521 H. Tianou, W. Wang, X. Yang, Z. Cao, Q. Kuang, Z. Wang, Z. Shan, M. Jin and Y. Yin, Nat. Commun., 2017, 8, 1261.

522 S. Zhou, M. Chen, Q. Lu, J. Hu, H. Wang, K. Li, K. Li, J. Zhang, Z. Zhu and Q. Liu, Mater. Lett., 2019, 247, 15-18.

523 W. Xin, T. Gao, W. Zhang, T. Hu, X. Sun and G. Zhou, J. Alloys Compd., 2019, 784, 157-164.

524 D. Zhang, Z. Yang, Z. Wu and G. Dong, Sens. Actuators, B, 2019, 283, 42-51.

525 T. Zhang, J. Zheng, Z. Liang, B. Zhao, H. Zeng, W. Guo, L. Zhao, Y. Sun, I. Abdulhalim and L. Jiang, Electrochim. Acta, 2019, 306, 151-158. 
526 B. Dong, W. Li, X. Huang, Z. Ali, T. Zhang, Z. Yang and Y. Hou, Nano Energy, 2019, 55, 37-41.

527 R. Ghosh Chaudhuri and S. Paria, Chem. Rev., 2012, 112, 2373-2433.

528 W. Schärtl, Nanoscale, 2010, 2, 829.

529 L. León Félix, J. A. H. Coaquira, M. A. R. Martínez, G. F. Goya, J. Mantilla, M. H. Sousa, L. D. L. S. Valladares, C. H. W. Barnes and P. C. Morais, Sci. Rep., 2017, 7, 41732. 530 S. Wei, Q. Wang, J. Zhu, L. Sun, H. Lin and Z. Guo, Nanoscale, 2011, 3, 4474.

531 F. Forato, S. Talebzadeh, N. Rousseau, J.-Y. Mevellec, B. Bujoli, D. A. Knight, C. Queffélec and B. Humbert, Phys. Chem. Chem. Phys., 2019, 21, 3066-3072.

532 H. Mi, X. Zhang, S. An, X. Ye and S. Yang, Electrochem. Commun., 2007, 9, 2859-2862.

533 R. M. Abdel Hameed, New J. Chem., 2018, 42, 2658-2668.

534 M.-Y. Kuo, C.-F. Hsiao, Y.-H. Chiu, T.-H. Lai, M.-J. Fang, J.-Y. Wu, J.-W. Chen, C.-L. Wu, K.-H. Wei, H.-C. Lin and Y.-J. Hsu, Appl. Catal., B, 2019, 242, 499-506.

535 H. S. Choi, J. H. Park, J. W. Bae, J. H. Lee and T. S. Chang, Catal. Commun., 2019, 123, 86-90.

536 K. Awazu, M. Fujimaki, C. Rockstuhl, J. Tominaga, H. Murakami, Y. Ohki, N. Yoshida and T. Watanabe, J. Am. Chem. Soc., 2008, 130, 1676-1680.

537 S. V. Salihov, Y. A. Ivanenkov, S. P. Krechetov, M. S. Veselov, N. V. Sviridenkova, A. G. Savchenko, N. L. Klyachko, Y. I. Golovin, N. V. Chufarova, E. K. Beloglazkina and A. G. Majouga, J. Magn. Magn. Mater., 2015, 394, 173-178.

538 H. Wang, Q. Mu, R. Revia, K. Wang, B. Tian, G. Lin, W. Lee, Y.-K. Hong and M. Zhang, J. Controlled Release, 2018, 289, 70-78.

539 K. C. Kwon, J. H. Ryu, J.-H. Lee, E. J. Lee, I. C. Kwon, K. Kim and J. Lee, Adv. Mater., 2014, 26, 6436-6441.

540 Y.-K. Kim, H.-K. Na, S. Kim, H. Jang, S.-J. Chang and D.-H. Min, Small, 2015, 11, 2527-2535.
541 A. Seth, H. Gholami Derami, P. Gupta, Z. Wang, P. Rathi, R. Gupta, T. Cao, J. J. Morrissey and S. Singamaneni, ACS Appl. Mater. Interfaces, 2020, 12, 42499-42510.

542 Y. Xiang, X. Peng, X. Kong, Z. Tang and H. Quan, Colloids Surf., A, 2020, 594, 124652.

543 Q. Lv, H. Min, D. Duan, W. Fang, G. Pan, A. Shen, Q. Wang, G. Nie and J. Hu, Adv. Healthcare Mater., 2018, 8, 1801257.

544 L. Li, Y. Lu, C. Jiang, Y. Zhu, X. Yang, X. Hu, Z. Lin, Y. Zhang, M. Peng, H. Xia and C. Mao, Adv. Funct. Mater., 2018, 28, 1704623.

545 T. Liu, X. Li, J. Wang, P. Zhang, X. Huang, Z. Zhang, D.-S. Guo and X. Yang, J. Mater. Chem. B, 2020, 8, 5483-5490.

546 K.-C. Ho and L.-Y. Lin, J. Mater. Chem. A, 2019, 7, 3516-3530.

547 Y. Bai, R. Zhang, X. Ye, Z. Zhu, H. Xie, B. Shen, D. Cai, B. Liu, C. Zhang, Z. Jia, S. Zhang, X. Li and F. Wei, Nat. Nanotechnol., 2018, 13, 589-595.

548 F. Yang, X. Wang, D. Zhang, J. Yang, D. Luo, Z. Xu, J. Wei, J.-Q. Wang, Z. Xu, F. Peng, X. Li, R. Li, Y. Li, M. Li, X. Bai, F. Ding and Y. Li, Nature, 2014, 510, 522-524.

549 J. Liu, J. Lu, X. Lin, Y. Tang, Y. Liu, T. Wang and H. Zhu, Comput. Mater. Sci., 2017, 129, 290-294.

550 C. R. Vandenabeele and S. Lucas, Mater. Sci. Eng., R, 2020, 139, 100521.

551 Y. He, W. Chen, X. Li, Z. Zhang, J. Fu, C. Zhao and E. Xie, ACS Nano, 2013, 7, 174-182.

552 C. Li and G. Shi, Nanoscale, 2012, 4, 5549.

553 P. Tucci, G. Porta, M. Agostini, D. Dinsdale, I. Iavicoli, K. Cain, A. Finazzi-Agró, G. Melino and A. Willis, Cell Death Dis., 2013, 4, e549-e549.

554 T. G. Smijs and S. Pavel, Nanotechnol., Sci. Appl., 2011, 4, 95-112.

555 W. J. Stark, P. R. Stoessel, W. Wohlleben and A. Hafner, Chem. Soc. Rev., 2015, 44, 5793-5805. 